University of Tennessee Health Science Center

UTHSC Digital Commons

$11-2012$

\title{
Development of the Sustained Release Analgesic Formulations for Rodents and a Novel In Vitro Model for Parenteral Formulations with the Character of a Level A IVIVC
}

Jin Xu

University of Tennessee Health Science Center

Follow this and additional works at: https://dc.uthsc.edu/dissertations

Part of the Medicinal and Pharmaceutical Chemistry Commons, and the Pharmaceutics and Drug Design Commons

\section{Recommended Citation}

$\mathrm{Xu}$, Jin , "Development of the Sustained Release Analgesic Formulations for Rodents and a Novel In Vitro Model for Parenteral Formulations with the Character of a Level A IVIVC" (2012). Theses and Dissertations (ETD). Paper 296. http://dx.doi.org/10.21007/etd.cghs.2012.0362. 


\title{
Development of the Sustained Release Analgesic Formulations for Rodents and a Novel In Vitro Model for Parenteral Formulations with the Character of a Level A IVIVC
}

\author{
Abstract \\ Laboratory animals are often subjected to various painful surgical procedures such as laparotomy, \\ thoracotomy or orthopedic procedures as well as non-surgical procedures such as the induction of \\ arthritis. Any procedure that causes pain in humans is assumed to cause pain in animals too. It is the \\ ethical obligation of all research personnel to reduce or preferably eliminate pain and distress by using \\ analgesics. Furthermore, the Institutional Animal Care and Use Committee (IACUC) requires that \\ appropriate anesthetics and/or analgesics must be used to minimize or eliminate pain and distress for \\ animals undergoing painful procedures.
}

The oral administration route is the most convenient delivery method for humans. However, using the oral administration route for rodents, such as putting an analgesic in the water, runs the risk of inaccurate dosing and that of degradation due to hydrolysis. Therefore methods for analgesic delivery to rodents are primarily limited to parenteral rather than oral delivery.

Rodents are the most universally used laboratory animals and are often subjected to research involving painful procedures. A survey of current literature indicates that buprenorphine is the most widely used narcotic analgesic for rodents because of its excellent analgesic activity and long duration of action. The Formulary for Laboratory Animals lists the buprenorphine dose for rats as $0.05 \mathrm{mg} / \mathrm{kg}$ (SC, tid, qid). Other referenced buprenorphine doses for rats vary widely $(0.01-0.05 \mathrm{mg} / \mathrm{kg}, \mathrm{SC} / \mathrm{IV}$, tid, bid) and require multiple administrations. The recommended dose of buprenorphine for mice following laparotomy is 0.05 to 0.1 $\mathrm{mg} / \mathrm{kg}$, subcutaneously (SC) twice a day. Referenced buprenorphine doses for maintaining analgesia in mice vary widely $(0.05-2.5 \mathrm{mg} / \mathrm{kg}$, SC, IV) and repeated administration is required. However, no sustainedrelease analgesic product for rodents was available when we started the project in 2008 . Therefore, in order to reduce the stress of frequent handling and injection as well as improve the well-being of research animals, the first objective of this project was to develop a long-acting sustained release formulation of buprenorphine which is capable of maintaining analgesia in rats and mice for 3 to 5 days following a single subcutaneous administration.

Current United States Pharmacopeia (USP) apparatus for in vitro drug release testing was designed mainly for oral and transdermal products. In contrast, there are no standard regulatory methods for parenteral sustained release products at present. As regards to quality control as well as formulation development purposes, it is still highly desirable to develop a suitable in vitro release model for parenteral products with the characters of Level A In Vitro-In Vivo Correlation (IVIVC) and elaborate drug release mechanism. So far there is no successful in vitro dissolution method with the character of Level A IVIVC exists at present for parenteral oily formations. Only a few publications stated in vitro in vivo correction for parenteral product, but they were developed from only one particular formulation. In addition, for most examples in which IVIVC was stated for parenterals, the mathematical models that described the drug release had not been provided. Therefore, the second objective of this study was to (1) design and develop a new dissolution model with the character of Level A IVIVC for oily formulations, and (2) develop a mathematical equation to describe the drug release mechanism from the in vitro model. In general, it is accepted that the process of development and validation of IVVIC for parenterals could follow the same principles as modified release (MR) oral products. The principles of FDA IVIVC MR guidance for oral products were used to develop and validate level A IVIVC for parenterals in this study.

In order to develop sustained release analgesic formulations, nine hydrophobic vehicles, which have been used in the pharmaceutical products, were selected based on clogP values. Solubility and short-term 
stability studies were performed and six vehicles were selected as candidates according to the results. Since there is no validated in vitro dissolution model for sustained release parenterals at present, a new in vitro dissolution model were designed based on the in vivo drug absorption mechanism after subcutaneous injection. In addition, a mathematical equation was proposed and validated to describe the drug release from this dissolution system. In order to apply this dissolution model to screen the parenteral formulations in vitro, Level A IVIVC study were performed. In-vivo release of buprenorphine from two different oily solutions was performed with subcutaneous administration in rats. Loo-Reigelman method was used to deconvolute plasma data. The mathematical equation, proposed to describe the in vitro drug release profiles, was also corresponded well with the in-vivo deconvolution data for the tested formulations ( $R 2>0.99$ ). Good linear correlations ( $R 2>0.99$ ) were also obtained between the mean percentage of drug absorbed and the mean percentage of drug dissolved. Internal predictability showed that absolute predictive error (\%PE) of Cmax and AUC were $4.9 \%$ and $0.9 \%, 12.6 \%$ and $3.4 \%$ for the tested formulations, respectively. External predictability showed that absolute predictive error (\%PE) of Cmax and AUC were $11.9 \%$ and $1.1 \%$. This new designed in vitro dissolution apparatus was used to screen six oily vehicles and TBC was selected. After subcutaneous injection of TBC formulation at the dose of 1.5 $\mathrm{mg} / \mathrm{Kg}$ in rats, $55 \%$ of maximum possible effect (MPE) analgesia can be maintained for 3 days and $20 \%$ MPE for 5 days in conventional tail flick model. The same formulation was tested in mice at the dose of $2.2 \mathrm{mg} / \mathrm{Kg}$ which can maintain more than $30 \%$ MPE analgesia for 3 days and at the dose of $8.9 \mathrm{mg} / \mathrm{Kg}$ which can keep at least $47 \%$ MPE analgesia for 4 days.

\section{Document Type}

Dissertation

Degree Name

Doctor of Philosophy (PhD)

\section{Program}

Pharmaceutical Sciences

\section{Research Advisor}

James R. Johnson

\section{Keywords}

Accelerated study, Dissolution model, Formulation, IVIVC, Mechanism, Sustained release

\section{Subject Categories}

Medicinal and Pharmaceutical Chemistry | Medicine and Health Sciences | Pharmaceutics and Drug Design | Pharmacy and Pharmaceutical Sciences 
Development of the Sustained Release Analgesic Formulations for Rodents and a Novel In Vitro Model for Parenteral Formulations with the Character of a Level A IVIVC

\author{
A Dissertation \\ Presented for \\ The Graduate Studies Council \\ The University of Tennessee \\ Health Science Center
}

\author{
In Partial Fulfillment \\ Of the Requirements for the Degree \\ Doctor of Philosophy \\ From The University of Tennessee
}

By

Jin $\mathrm{Xu}$

December 2012 
Copyright (C) 2012 by Jin Xu.

All rights reserved. 


\section{DEDICATION}

I dedicate this work to my parents, Mr. Yichun Xu and Mrs. Xiuying Xia, for their love and support. 


\section{ACKNOWLEDGEMENTS}

My sincerest gratitude goes to my major advisor Dr. James R. Johnson who guided, advised and supported me during my Ph.D. work. I would extend special thanks and acknowledgement to Dr. Atul J. Shukla for the opportunity, guidance, advice and support during the research work.

I would also like to thank my committee members, Dr. Isaac O. Donkor, Dr. Tim Mandrell, Dr. Arthur D. Straughn and Dr. George C. Wood for their invaluable guidance, suggestions and help in my work.

I further would like to thank my group members, Mr. Ed Brunson, Dr. Wen Qu, Dr. Paras Jain, Dr. Weiqiang Cheng, Rasika Suryawanshi, Dr. Sonia Bedi, Dr. Yinqi Zhou, Dr. Suresh Potharaju, Qiuye Zhang, Rui Zhu, Dr. Wenli Lu and Dr. Namrata Trivedi, for their continuous help and support. This is a really great group and they have made my research at UT much easier. Here I learned how to work independently and work as a team member. In addition, I sincerely thank Dr. Tim Mandrell and Ms. Lillian Zalduondo for the help in my animal research.

I would also like to thank Ms. Connie L. Burk from Office of International Affairs for her continuous help in the last eight years. Also I would like to thank Ms. Shirley Hancock for her help and suggestions for the formatting of my dissertation. I want to thank University of Tennessee Health Science Center for providing me this wonderful opportunity and financial support, and all the faculty and staff in Department of Pharmaceutical Sciences for their help and support during my graduate study.

Most of all, I would like to express my deepest appreciation to my parents, $\mathrm{Mr}$. Yichun $\mathrm{Xu}$ and Ms. Xiuying Xia, and my sister, Jie $\mathrm{Xu}$, for the continuous encouragement, support and love.

This work was supported by American College of Laboratory Animal Medicine (ACLAM) Foundation and grant SBIR R43 RR24079-01A1. 


\begin{abstract}
Laboratory animals are often subjected to various painful surgical procedures such as laparotomy, thoracotomy or orthopedic procedures as well as non-surgical procedures such as the induction of arthritis. Any procedure that causes pain in humans is assumed to cause pain in animals too. It is the ethical obligation of all research personnel to reduce or preferably eliminate pain and distress by using analgesics. Furthermore, the Institutional Animal Care and Use Committee (IACUC) requires that appropriate anesthetics and/or analgesics must be used to minimize or eliminate pain and distress for animals undergoing painful procedures.
\end{abstract}

The oral administration route is the most convenient delivery method for humans. However, using the oral administration route for rodents, such as putting an analgesic in the water, runs the risk of inaccurate dosing and that of degradation due to hydrolysis. Therefore methods for analgesic delivery to rodents are primarily limited to parenteral rather than oral delivery.

Rodents are the most universally used laboratory animals and are often subjected to research involving painful procedures. A survey of current literature indicates that buprenorphine is the most widely used narcotic analgesic for rodents because of its excellent analgesic activity and long duration of action. The Formulary for Laboratory Animals lists the buprenorphine dose for rats as $0.05 \mathrm{mg} / \mathrm{kg}$ (SC, tid, qid). Other referenced buprenorphine doses for rats vary widely $(0.01-0.05 \mathrm{mg} / \mathrm{kg}, \mathrm{SC} / \mathrm{IV}$, tid, bid) and require multiple administrations. The recommended dose of buprenorphine for mice following laparotomy is 0.05 to $0.1 \mathrm{mg} / \mathrm{kg}$, subcutaneously (SC) twice a day. Referenced buprenorphine doses for maintaining analgesia in mice vary widely $(0.05-2.5 \mathrm{mg} / \mathrm{kg}, \mathrm{SC}$, IV) and repeated administration is required. However, no sustained-release analgesic product for rodents was available when we started the project in 2008. Therefore, in order to reduce the stress of frequent handling and injection as well as improve the well-being of research animals, the first objective of this project was to develop a long-acting sustained release formulation of buprenorphine which is capable of maintaining analgesia in rats and mice for 3 to 5 days following a single subcutaneous administration.

Current United States Pharmacopeia (USP) apparatus for in vitro drug release testing was designed mainly for oral and transdermal products. In contrast, there are no standard regulatory methods for parenteral sustained release products at present. As regards to quality control as well as formulation development purposes, it is still highly desirable to develop a suitable in vitro release model for parenteral products with the characters of Level A In Vitro-In Vivo Correlation (IVIVC) and elaborate drug release mechanism. So far there is no successful in vitro dissolution method with the character of Level A IVIVC exists at present for parenteral oily formations. Only a few publications stated in vitro in vivo correction for parenteral product, but they were developed from only one particular formulation. In addition, for most examples in which IVIVC was stated for parenterals, the mathematical models that described the drug release had not been provided. Therefore, the second objective of this study was to (1) design and 
develop a new dissolution model with the character of Level A IVIVC for oily formulations, and (2) develop a mathematical equation to describe the drug release mechanism from the in vitro model. In general, it is accepted that the process of development and validation of IVVIC for parenterals could follow the same principles as modified release (MR) oral products. The principles of FDA IVIVC MR guidance for oral products were used to develop and validate level A IVIVC for parenterals in this study.

In order to develop sustained release analgesic formulations, nine hydrophobic vehicles, which have been used in the pharmaceutical products, were selected based on clogP values. Solubility and short-term stability studies were performed and six vehicles were selected as candidates according to the results. Since there is no validated in vitro dissolution model for sustained release parenterals at present, a new in vitro dissolution model were designed based on the in vivo drug absorption mechanism after subcutaneous injection. In addition, a mathematical equation was proposed and validated to describe the drug release from this dissolution system. In order to apply this dissolution model to screen the parenteral formulations in vitro, Level A IVIVC study were performed. Invivo release of buprenorphine from two different oily solutions was performed after subcutaneous administration in rats. Loo-Reigelman method was used to deconvolute plasma data. The mathematical equation, proposed to describe the in vitro drug release profiles, was also corresponded well with the in-vivo deconvolution data for the tested formulations $\left(\mathrm{R}^{2}>0.99\right)$. Good linear correlations $\left(\mathrm{R}^{2}>0.99\right)$ were also obtained between the mean fraction of drug absorbed and the mean percentage of drug dissolved. Internal predictability showed that absolute predictive error (\%PE) of Cmax and AUC were $4.9 \%$ and $0.9 \%, 12.6 \%$ and $3.4 \%$ for the tested formulations, respectively. External predictability showed that absolute predictive error $(\% \mathrm{PE})$ of Cmax and AUC were $11.9 \%$ and $1.1 \%$. This new designed in vitro dissolution apparatus was used to screen six oily vehicles and TBC was selected. After subcutaneous injection of TBC formulation at the dose of $1.5 \mathrm{mg} / \mathrm{Kg}$ in rats, $55 \%$ of maximum possible effect (MPE) analgesia can be maintained for 3 days and 20\% MPE for 5 days in conventional tail flick model. The same formulation was tested in mice at the dose of $2.2 \mathrm{mg} / \mathrm{Kg}$ which can maintain more than $30 \%$ MPE analgesia for 3 days and at the dose of $8.9 \mathrm{mg} / \mathrm{Kg}$ which can keep at least $47 \%$ MPE analgesia for 4 days. 


\section{TABLE OF CONTENTS}

CHAPTER 1. INTRODUCTION .....................................................................................1

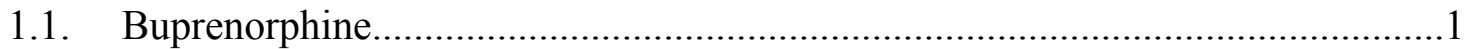

1.1.1. Description ......................................................................................

1.1.2. Current commercial dosage forms of buprenorphine in human .................... 1

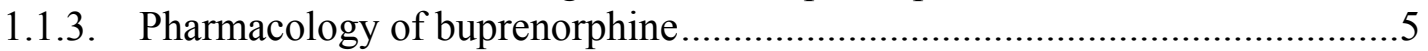

1.1.4. Pharmacokinetics of buprenorphine ....................................................... 7

1.1.4.1. Pharmacokinetic parameters ...............................................................

1.1.4.2. Absorption distribution metabolism and excretion (ADME) ....................7

1.2. Injectable Analgesics for Rodents ..............................................................

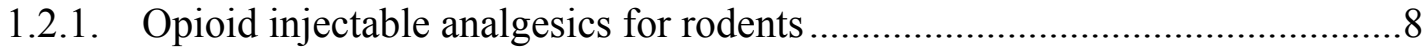

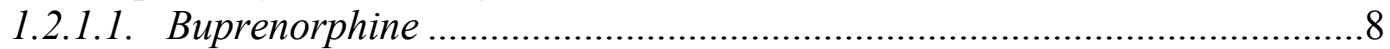

1.2.1.2. Morphine ...................................................................................... 8

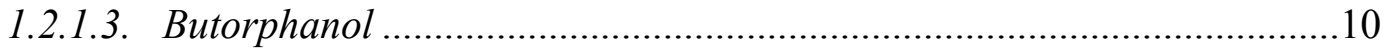

1.2.2. NSAID injectable analgesics for rodents ............................................. 10

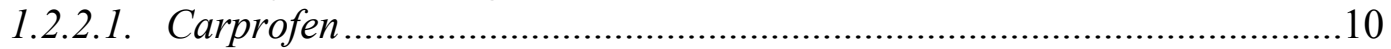

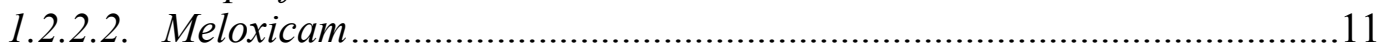

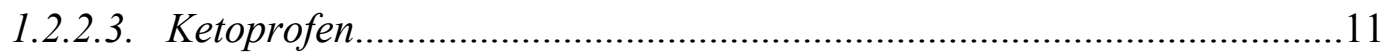

1.3. Sustained Release Analgesic Formulations in Animals.................................... 12

1.4. In Vitro Dissolution Model for Parenteral Dosage Forms .................................12

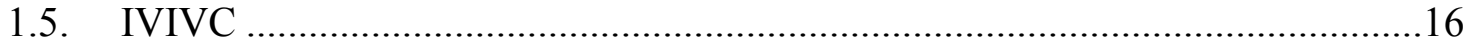

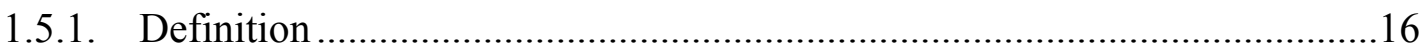

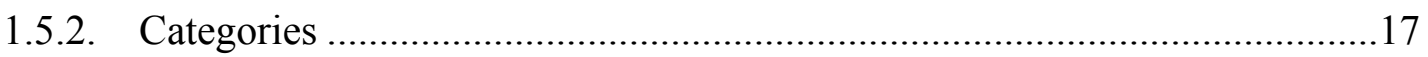

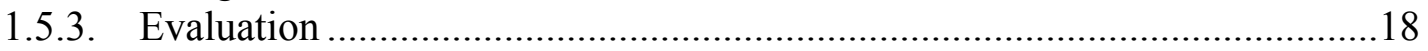

\section{CHAPTER 2. DEVELOPMENT OF A SUSTAINED RELEASE} BUPRENORPHINE FORMULATION FOR MAINTAINING PROLONGED

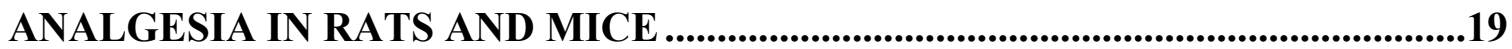

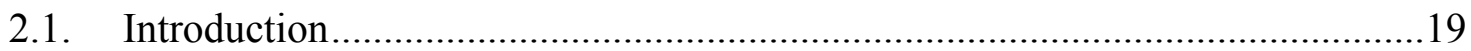

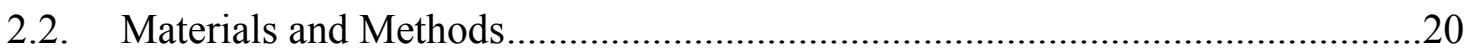

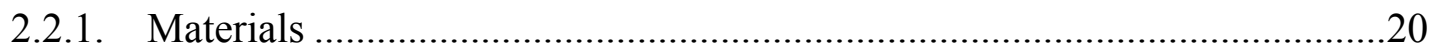

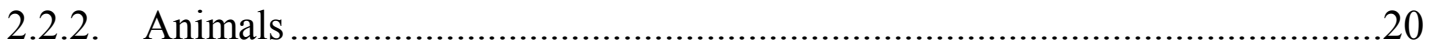

2.2.3. Analytical methodology for in vitro and in vivo studies ...........................20

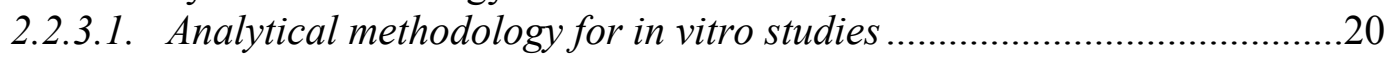

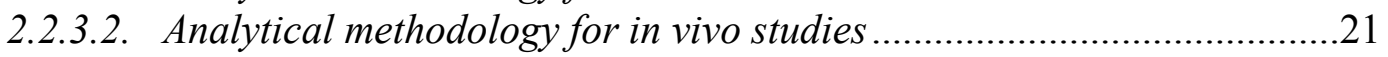

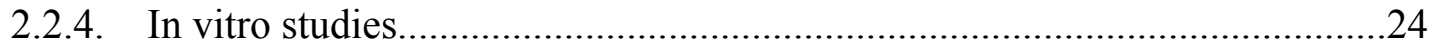

2.2.4.1. Determination of equilibrium solubility of buprenorphine ....................24

2.2.4.2. Determination of stability of buprenorphine in various solvents ...........24

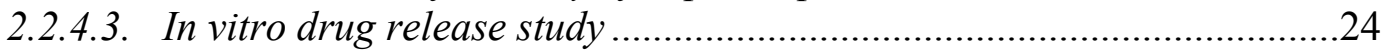

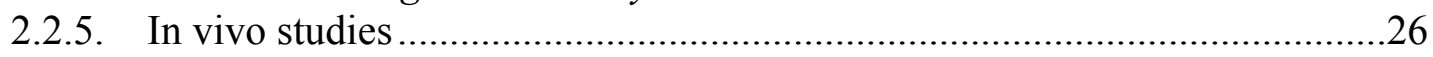

2.2.5.1. Measurement of analgesia in rats and mice using the tail flick

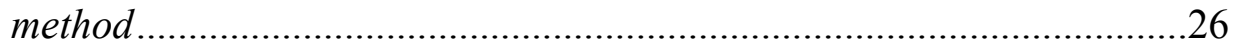

2.2.5.2. $P K$ and $P D$ studies of buprenorphine in rats after an intravenous injection of buprenorphine solution .....................................................22 
2.2.5.3. $P K$ and PD studies of buprenorphine in rats after a subcutaneous injection of buprenorphine sustained release formulations...................27

2.2.5.4. PK and PD studies of buprenorphine in mice after subcutaneous injection of buprenorphine sustained release formulations...................29

2.2.5.5. Toxicity evaluation of citric acid esters in rats.....................................29

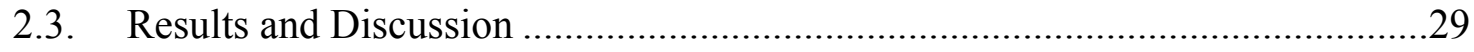

2.3.1. Validation of analytical methodology ...................................................29

2.3.1.1. Validation of HPLC assay for in vitro studies ...................................29

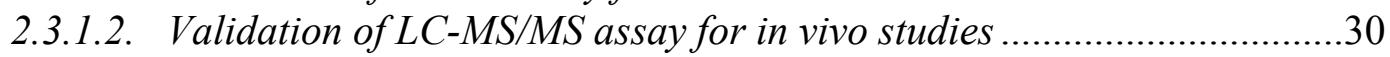

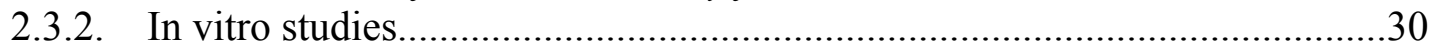

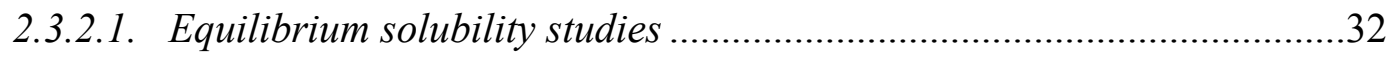

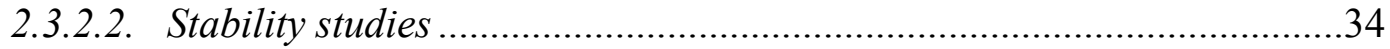

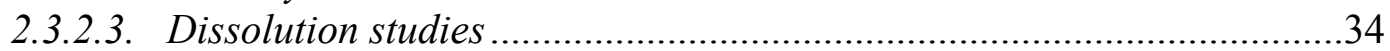

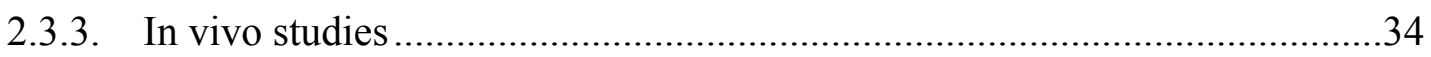

2.3.3.1. PK and PD studies in rats after intravenous injection of buprenorphine saline solution ...........................................................34

2.3.3.2. $P K$ and $P D$ studies in rats after subcutaneous injection of buprenorphine sustained release formulations.....

2.3.3.3. In vivo evaluation of buprenorphine sustained release formulations in mice

2.3.3.4. Typical photographs of the subcutaneous tissue at the injection site after administration of different formulations in rats and mice

2.2.3.5. Evaluation of toxicity of citric acid esters in rats ..................................44

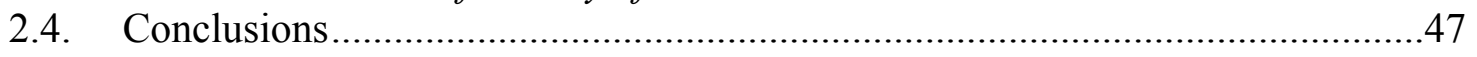

\section{CHAPTER 3. A NOVEL IN VITRO DISSOLUTION MODEL FOR OILY FORMULATIONS: MODEL DESIGN, RELEASE MECHANISM, AND THE

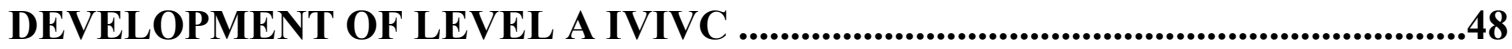

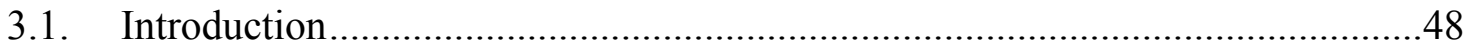

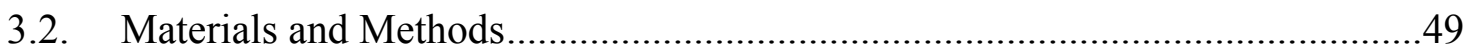

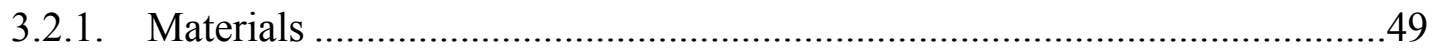

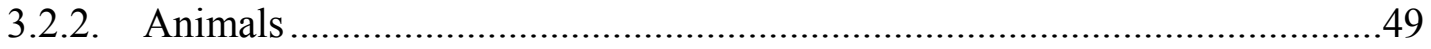

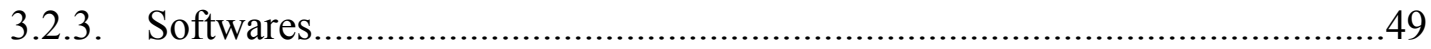

3.2.4. Analytical methodology for in vitro and in vivo studies ...........................49

3.2.4.1. Analytical methodology for in vitro studies - HPLC/fluorescence

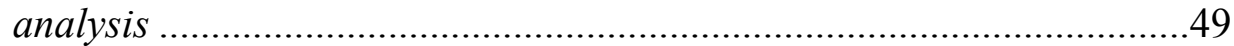

3.2.4.2. Analytical methodology for in vivo studies - LC-MS/MS method ..........50

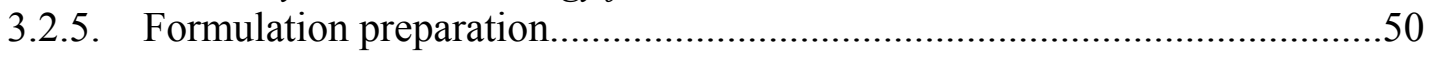

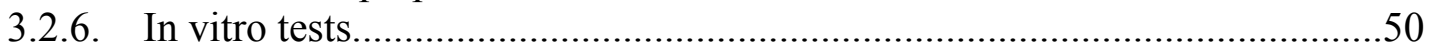

3.2.6.1. In vitro dissolution model design ......................................................50

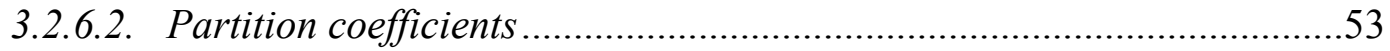

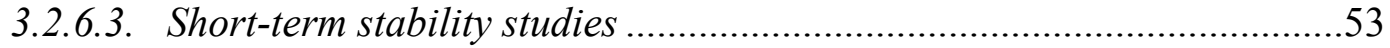

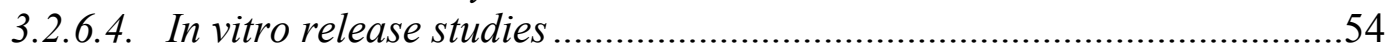

3.2.6.5. Discriminating power of the dissolution test ......................................54

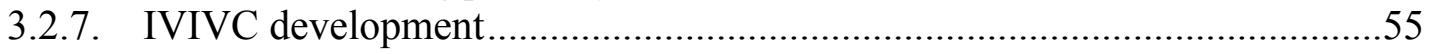

3.2.7.1. In vitro studies for IVIVC development ..............................................55 
3.2.7.2. In vivo evaluation of buprenorphine solutions in rats for IVIVC

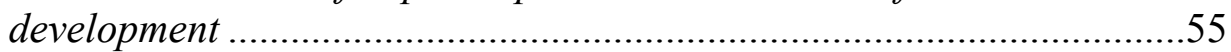

3.2.7.3. Bioavailability of buprenorphine in rats............................................56

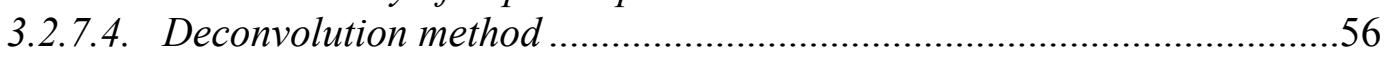

3.2.7.5. IVIVC development and validation .....................................................57

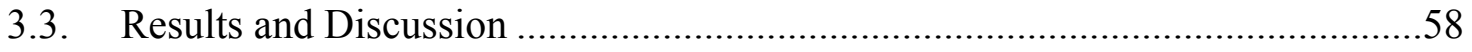

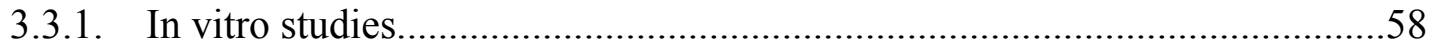

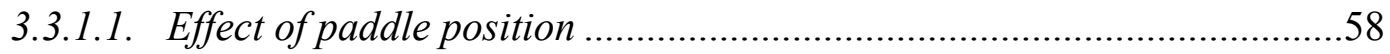

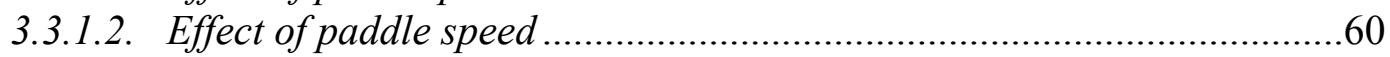

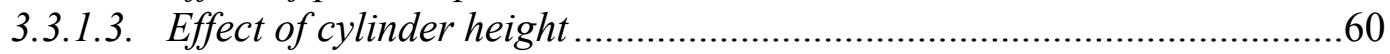

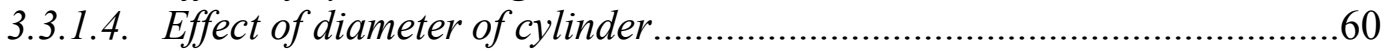

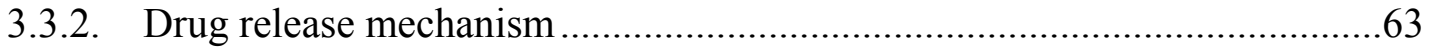

3.3.2.1. Derivation of mathematical Equation .....................................................65

3.3.2.2. Verification of mathematical equation....................................................68

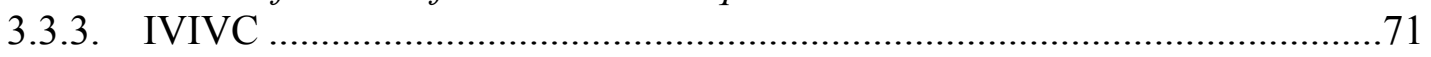

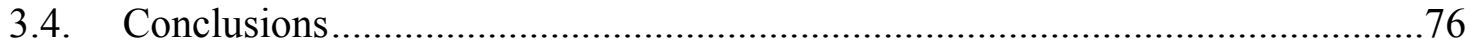

\section{CHAPTER 4. APPLICATION OF A NOVEL IN VITRO DISSOLUTION MODEL: A RATIONAL METHOD TO SELECT THE DISSOLUTION CONDITIONS IN AN ACCELERATED DISSOLUTION STUDY............................83}

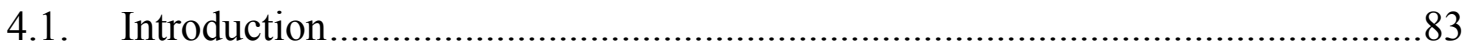

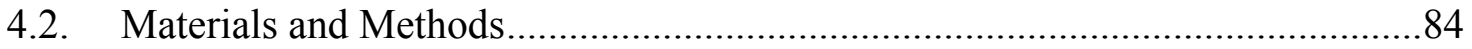

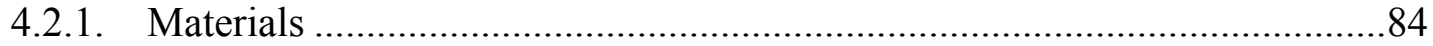

4.2.2. Analytical methodology - HPLC/Fluorescence analyses ..............................84

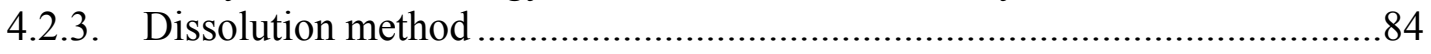

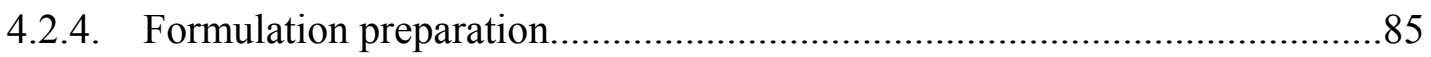

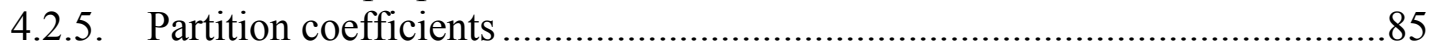

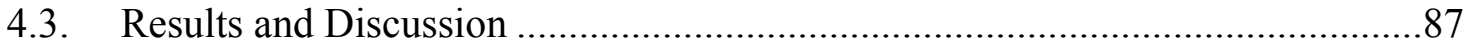

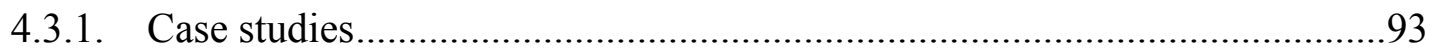

4.3.1.1. Case 1 (Formulation 4-1, time scale factor $=7$ ) ..................................93

4.3.2.2. Case 2 (Formulation 4-2, time scale factor $=14$ ) ..................................94

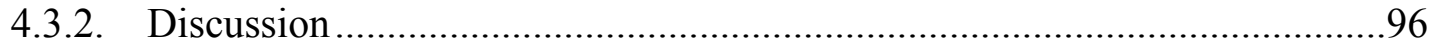

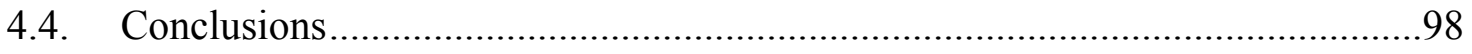

LIST OF REFERENCES ....................................................................................................101

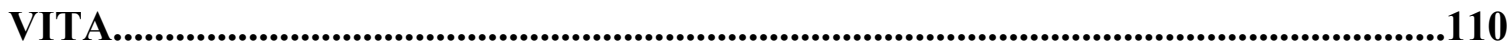




\section{LIST OF TABLES}

Table 1-1. Commercially available buprenorphine products. ......................................3

Table 1-2. Apparent $\mathrm{K}_{\mathrm{i}}$ values of buprenorphine for the various members of the opioid receptor family.....................................................................6

Table 1-3. Recommended analgesics for rats and mice...........................................

Table 2-1. Buprenorphine sustained release formulations for in vitro dissolution

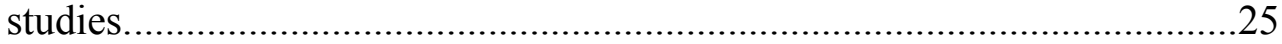

Table 2-2. Buprenorphine sustained release formulations tested in rats......................28

Table 2-3. Buprenorphine sustained release formulations tested in mice.....................28

Table 2-4. Intra-day $(n=3)$ and inter-day $(n=3)$ variation of the assay $1 \ldots \ldots \ldots \ldots \ldots \ldots . . . . . . . .11$

Table 2-5. Intra-day $(n=5)$ and inter-day $(n=3)$ variation of the assay $2 \ldots \ldots \ldots \ldots \ldots . . . . . .31$

Table 2-6. Intra-day and Inter-day variation of the LC-MS/MS assay $(n=3) \ldots \ldots \ldots \ldots . . .31$

Table 2-7. Solubility of buprenorphine in different vehicles. .....................................33

Table 2-8. Stability of buprenorphine base in different solvents. ..............................36

Table 2-9. PK parameters of buprenorphine in rats after a single intravenous injection at the dose of $0.05 \mathrm{mg} / \mathrm{kg}$......................................................4

Table 3-1. The formulations tested in the dissolution model development study..........51

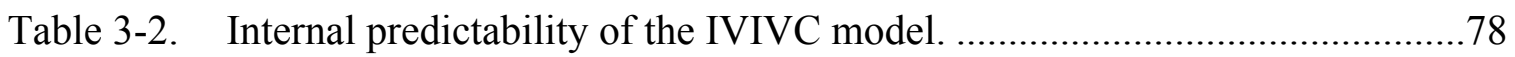

Table 3-3. External predictability of the IVIVC model ..........................................78

Table 4-1. Formulations tested in the method development of accelerated dissolution study.

Table 4-2. Dissolution paddle speed, partition coefficient and calculated parameters in accelerated studies. 


\section{LIST OF FIGURES}

Figure 1-1. Chemical structure, molecular weight, empirical formula, CAS Number and chemical name of buprenorphine.

Figure 1-2. Schematic illustration of different in vitro dissolution methods for parenterals.

Figure 2-1. Stability of buprenorphine in various solvents.

Figure 2-2. In vitro release profile of buprenorphine from oily formulations prepared with various hydrophobic vehicles.

Figure 2-3. (a) Analgesic effect and (b) plasma concentration of buprenorphine in rats after a single intravenous injection of buprenorphine hydrochloride solution at the dose of $0.05 \mathrm{mg} / \mathrm{kg}$.

Figure 2-4. Effect of varying buprenorphine dose on (a) analgesia and (b) buprenorphine plasma concentration in rats after a single subcutaneous injection of the formulations prepared with TBC.

Figure 2-5. Effect of doses on (a) analgesia $(\mathrm{n}=5)$ and (b) buprenorphine plasma concentrations in mice after subcutaneous injection of formulations.

Figure 2-6. Typical injection site after subcutaneous administration of buprenorphine formulation prepared with TBC in (a) rats and (b) mice at day 5 .

Figure 2-7. Body weight changes of rats after subcutaneous administration of different citric acid esters or normal saline solution.

Figure 3-1. (a) Cylinder and (b) cylinder-in-basket (CIB) apparatus for in vitro dissolution study.

Figure 3-2. The effect of paddle position on the release amount of buprenorphine in CIB dissolution model.

Figure 3-3. The effect of paddle speed on the release amount of buprenorphine in CIB dissolution model

Figure 3-4. The effect of cylinder height on the release amount of buprenorphine in CIB dissolution model.

Figure 3-5. The effect of cylinder diameter on the release amount of buprenorphine in CIB dissolution model. 
Figure 3-6. (a) The release profile of buprenorphine from formulation 3-1c, and predicted data and experimental data generated from (b1) cylinder \#1 and (b2) \#3.

Figure 3-7. The buprenorphine release profile and the comparison of experimental data and predicted data at different conditions.

Figure 3-8. In vivo data process of formulation 3-1b and 3-2 after subcutaneous injection in rats for IVIVC development

Figure 3-9. In vitro data process of formulation 3-1b and 3-2 for IVIVC development

Figure 3-10. IVIVC model linear regression plots of percent absorbed versus percent released for formulation 3-1b and 3-2.

Figure 3-11. In vitro drug release of formulation 3-3a.

Figure 3-12. (a) Plasma concentration profile of buprenorphine, and (b) in vivo cumulative drug release profile after s.c. injection of formulation 3-3a in rats.

Figure 3-13. Linear regression of percent absorbed versus percent released for formulation 3-3a.

Figure 4-1. In vitro drug release profiles from (a) formulation 4-1 and (b) formulation 4-2 in USP specified dissolution media at different $\mathrm{pH}$ .88

Figure 4-2. The procedures of selecting dissolution medium in accelerated study.........91

Figure 4-3. Predicted drug release amount in accelerated study and IVIVC (case 1).....95

Figure 4-4. Predicted drug release amount in accelerated study and IVIVC (case 2).....97

Figure 4-5. In vitro drug release from (a) formulation 4-1 and (b) formulation 4-2 in phosphate buffer dissolution media with different amount of SDS at $\mathrm{pH}$ 6 .

Figure 4-6. In vitro drug release from (a) formulation 4-1 and (b) formulation 4-2 in phosphate buffer dissolution media with different amount of SDS at $\mathrm{pH}$ 7.4 


\section{LIST OF ABBREVIATIONS}

\begin{tabular}{|c|c|}
\hline$\% \mathrm{PE}$ & the percent prediction error \\
\hline $\mathrm{ACN}$ & acetonitrile \\
\hline ANOVA & analysis of variance \\
\hline ATBC & acetyl tributyl citrate \\
\hline ATEC & acetyl triethyl citrate \\
\hline AUC & area under the curve \\
\hline bid & twice daily \\
\hline $\mathrm{CE}$ & collision energy \\
\hline $\mathrm{CF}$ & continuous flow \\
\hline CIB & cylinder-in-basket \\
\hline $\mathrm{COX}$ & cyclooxygenase \\
\hline CXP & collision cell exit potential \\
\hline DMB & dialysis membrane-based \\
\hline DP & declustering potential \\
\hline ER & extended release \\
\hline f1 & the difference factor \\
\hline $\mathrm{f} 2$ & the similarity factor \\
\hline $\mathrm{Fa}$ & the fraction of drug absorbed \\
\hline FDA & Food and Drug Administration \\
\hline $\mathrm{HCl}$ & hydrochloride \\
\hline HPLC & high-performance liquid chromatography \\
\hline $\mathrm{hr}$ & hour \\
\hline IACUC & the Institutional Animal Care and Use Committee \\
\hline im or IM & intramuscular \\
\hline IPA & isopropyl alcohol \\
\hline IV & intrinsic viscosity \\
\hline iv & intravenous \\
\hline IVIVC & In Vitro-In Vivo Correlation \\
\hline $\mathrm{kg}$ & kilogram \\
\hline LD50 & lethal dose, $50 \%$ \\
\hline LLOD & lower limit of detection \\
\hline LLOQ & lower limit of quantification \\
\hline
\end{tabular}




\begin{tabular}{|c|c|}
\hline $\mathrm{mcg}$ & micrograms \\
\hline $\mathrm{mg}$ & milligrams \\
\hline $\mathrm{mL}$ & milliliter \\
\hline MRM & multiple-reaction-monitoring \\
\hline NA & not available \\
\hline $\mathrm{NaCl}$ & Sodium chloride \\
\hline $\mathrm{NaOH}$ & Sodium hydroxide \\
\hline$n P A$ & n-propyl alcohol \\
\hline NSAIDs & non-steroidal anti-inflammatory drugs \\
\hline ORL & opiate receptor-like \\
\hline PBS & phosphate buffer saline \\
\hline PD & pharmacodynamic \\
\hline PEG & polyethylene glycol \\
\hline PK & Pharmacokinetic \\
\hline $\mathrm{PO}$ & by mouth (per os) \\
\hline q & every \\
\hline QC & quality control \\
\hline qid & four times daily \\
\hline $\mathrm{sc}$ or $\mathrm{SC}$ & subcutaneously \\
\hline SD & sprague dawley \\
\hline SEM & standard error of the mean \\
\hline sid & once daily \\
\hline SS & sample-and-separate \\
\hline $\mathrm{TBC}$ & tributyl citrate \\
\hline TEC & triethyl citrate \\
\hline tid & three times daily \\
\hline US & United State \\
\hline USP & United States Pharmacopeia \\
\hline VAP & vascular access ports \\
\hline
\end{tabular}




\section{CHAPTER 1. INTRODUCTION}

\subsection{Buprenorphine}

\subsubsection{Description}

Buprenorphine is a $\mu$-opioid receptor partial agonist and a $\kappa$-opioid receptor antagonist listed in Schedule III of the Controlled Substances Act. ${ }^{1}$ It is a semi-synthetic opioid derived from thebaine. ${ }^{2}$ It was first marketed in the 1980 s by Reckitt \& Colman (now Reckitt Benckiser) as an analgesic. The chemical structure of buprenorphine (Figure 1-1) resembles that of morphine, but it contains a cyclopropylmethyl group on the nitrogen atom. Buprenorphine base is a white or almost white powder and is slightly soluble in water, freely soluble in acetone, soluble in methanol and ether and slightly soluble in cyclohexane. The pKa values of buprenorphine are 8.5 and $10.0 .^{3}$

\subsubsection{Current commercial dosage forms of buprenorphine in human}

Current commercially marketed products of buprenorphine in humans include Buprenex injection, Suboxone tablet, Subutex tablet, Suboxone film, Transtec ER patch, Butrans ER patch, Norspan ER patch and Probuphine implant (Table 1-1).

Buprenex $^{1}$ is a parenteral opioid analgesic intended for intravenous or intramuscular administration for the relief of moderate to severe pain. Each $\mathrm{mL}$ of Buprenex injection contains $0.324 \mathrm{mg}$ buprenorphine hydrochloride (equivalent to $0.3 \mathrm{mg}$ buprenorphine), $50 \mathrm{mg}$ anhydrous dextrose, water for injection and $\mathrm{HCl}$ to adjust $\mathrm{pH}$.

Both Subutex $^{1}$ tablet and Suboxone ${ }^{1}$ tablet are indicated for the treatment of opioid dependence by sublingual administration because of the poor oral bioavailability of buprenorphine. Subutex contains only buprenorphine hydrochloride with other excipients, lactose, mannitol, cornstarch, povidone K30, citric acid, sodium citrate and magnesium stearate. Suboxone contains an additional ingredient, naloxone, with other excipients, including lactose, mannitol, cornstarch, povidone K30, citric acid, sodium citrate, FD\&C Yellow No.6 color, magnesium stearate, acesulfame K sweetener and a lemon/lime flavor. Naloxone is an opioid antagonist and is used in Suboxone tablet to deter the abuse of tablets by intravenous injection. Subutex and Suboxone are both available in $2 \mathrm{mg}$ and $8 \mathrm{mg}$ sublingual dosages.

Suboxone $^{1}$ is also approved by FDA on August 30, 2010 as a sublingual film in both the $2 \mathrm{mg} / 0.5 \mathrm{mg}$ and $8 \mathrm{mg} / 2 \mathrm{mg}$ dosages (buprenorphine/Naloxone). Reckitt Benckiser Pharmaceuticals claimed that the film has some advantages over the traditional tablet including dissolving faster, better taste, adhering to the oral mucosa under the tongue and preventing it from being swallowed or falling out. 


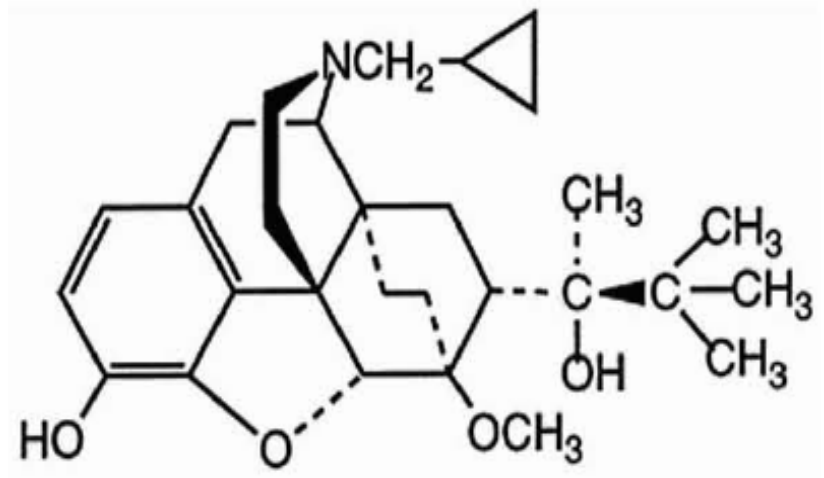

Molecular weight: $\quad 467.6$

Empirical formula: $\mathrm{C}_{29} \mathrm{H}_{41} \mathrm{NO}_{4}$.

CAS Number: $\quad$ 52485-79-7

Chemical name: (2s)-2-[17-cyclopropylmethyl)-4, 5a-epoxy-3-hydroxy-6-methoxy$6 \alpha, 14$-ethano-14 $\alpha$-morphinan-7 $\alpha$-yl]-3,3-dimethylbutan-2-ol

Figure 1-1. Chemical structure, molecular weight, empirical formula, CAS Number and chemical name of buprenorphine. 
Table 1-1. Commercially available buprenorphine products.

\begin{tabular}{|c|c|c|c|c|c|}
\hline $\begin{array}{l}\text { Trade } \\
\text { Name }\end{array}$ & $\begin{array}{c}\text { Dosage } \\
\text { Form/Route }\end{array}$ & Strength & Ingredients & Sponsor & $\begin{array}{l}\text { Approval } \\
\text { Date }\end{array}$ \\
\hline \multirow[t]{2}{*}{$\begin{array}{l}\text { Buprenex } \\
\text { (US) }\end{array}$} & \multirow[t]{2}{*}{$\begin{array}{l}\text { Injection Solution } \\
\text { /IV or IM }\end{array}$} & \multirow[t]{2}{*}{$0.3 \mathrm{mg}$ base $/ \mathrm{mL}$} & $\begin{array}{l}\text { buprenorphine } \mathrm{HCl}(0.324 \mathrm{mg} / \mathrm{mL}) \text {, } \\
\text { anhydrous dextrose }(50 \mathrm{mg} / \mathrm{mL}) \text {, } \\
\text { water for injection, } \mathrm{HCl} \text { (adjust } \mathrm{pH})\end{array}$ & $\begin{array}{l}\text { Reckitt Benkiser } \\
\text { Pharmaceuticals, } \\
\text { Inc. }\end{array}$ & $\begin{array}{l}\text { December 29, } \\
1981\end{array}$ \\
\hline & & & $\begin{array}{l}\text { NA } \\
\text { NA } \\
\text { NA }\end{array}$ & $\begin{array}{l}\text { Hospira (generic) } \\
\text { Bedford (generic) } \\
\text { Luitpold (generic) }\end{array}$ & $\begin{array}{l}\text { June 3, } 1996 \\
\text { March 2, } 2005 \\
\text { March 27, } 2007\end{array}$ \\
\hline $\begin{array}{l}\text { Suboxone } \\
\text { (US) }\end{array}$ & Tablet/Sublingual & $\begin{array}{l}2 \mathrm{mg} / 0.5 \mathrm{mg} \\
8 \mathrm{mg} / 2 \mathrm{mg} \\
\text { (buprenorphine } \\
\mathrm{HCl} / \text { Naloxone } \\
\mathrm{HCl} \text { ) }\end{array}$ & $\begin{array}{l}\text { buprenorphine } \mathrm{HCl} \text {, Naloxone } \mathrm{HCl} \text {, } \\
\text { lactose, mannitol, cornstarch, } \\
\text { povidone K30, citric acid, sodium } \\
\text { citrate, FD\&C Yellow No.6 color, } \\
\text { magnesium stearate, Acesulfame K } \\
\text { sweetener and a lemon lime flavor. }\end{array}$ & $\begin{array}{l}\text { Reckitt Benkiser } \\
\text { Pharmaceuticals, } \\
\text { Inc. }\end{array}$ & $\begin{array}{l}\text { October 8, } \\
2002\end{array}$ \\
\hline $\begin{array}{l}\text { Suboxone } \\
\text { (US) }\end{array}$ & Film/Sublingual & $\begin{array}{l}2 \mathrm{mg} / 0.5 \mathrm{mg} \\
8 \mathrm{mg} / 2 \mathrm{mg} \\
\text { (buprenorphine / } \\
\text { Naloxone) }\end{array}$ & $\begin{array}{l}\text { buprenorphine } \mathrm{HCl} \text {, naloxone } \mathrm{HCl} \\
\text { dihydrate, polyethylene oxide, } \\
\text { hydroxypropyl methylcellulose, } \\
\text { maltitol, acesulfame potassium, lime } \\
\text { flavor, citric acid, sodium citrate, } \\
\text { FD\&C yellow \#6, and white ink }\end{array}$ & $\begin{array}{l}\text { Reckitt Benkiser } \\
\text { Pharmaceuticals, } \\
\text { Inc. }\end{array}$ & $\begin{array}{l}\text { August 30, } \\
2010\end{array}$ \\
\hline \multirow[t]{4}{*}{$\begin{array}{l}\text { Subutex } \\
\text { (US) }\end{array}$} & \multirow[t]{4}{*}{ Tablet/Sublingual } & \multirow[t]{4}{*}{$2 \mathrm{mg} ; 8 \mathrm{mg}$ (base) } & $\begin{array}{l}\text { buprenorphine } \mathrm{HCl} \text {, lactose, } \\
\text { mannitol, cornstarch, povidone } \mathrm{K} 30 \text {, } \\
\text { citric acid, sodium citrate and } \\
\text { magnesium stearate. }\end{array}$ & $\begin{array}{l}\text { Reckitt Benkiser } \\
\text { Pharmaceuticals, } \\
\text { Inc. }\end{array}$ & $\begin{array}{l}\text { October } 8 \\
2002\end{array}$ \\
\hline & & & NA & $\begin{array}{l}\text { Roxane } \\
\text { Laboratories, Inc. } \\
\text { (generic) }\end{array}$ & $\begin{array}{l}\text { October } 8 \\
2009\end{array}$ \\
\hline & & & NA & $\begin{array}{l}\text { Barr Laboratories, } \\
\text { Inc. (generic) }\end{array}$ & May 7, 2010 \\
\hline & & & NA & $\begin{array}{l}\text { EthyPharm } \\
\text { (generic) }\end{array}$ & $\begin{array}{l}\text { September 24, } \\
2010\end{array}$ \\
\hline
\end{tabular}


Table 1-1. (Continued).

\begin{tabular}{|c|c|c|c|c|c|}
\hline $\begin{array}{l}\text { Trade } \\
\text { Name }\end{array}$ & $\begin{array}{c}\text { Dosage } \\
\text { Form/Route }\end{array}$ & Strength & Ingredients & Sponsor & $\begin{array}{l}\text { Approval } \\
\text { Date }\end{array}$ \\
\hline $\begin{array}{l}\text { Transtec } \\
\text { (US) }\end{array}$ & $\begin{array}{l}\text { ER patch (4-day) } \\
\text { /Transdermal }\end{array}$ & $\begin{array}{l}35,52.5 \text { and } 70 \\
\mathrm{mcg} / \mathrm{hr}\end{array}$ & NA & Grunenthal & 2001 \\
\hline $\begin{array}{l}\text { Butrans } \\
\text { (US) }\end{array}$ & $\begin{array}{l}\text { ER patch (7-day) } \\
\text { /Transdermal }\end{array}$ & 5,10 and $20 \mathrm{mcg} / \mathrm{hr}$ & NA & $\begin{array}{l}\text { Purdue Pharma, } \\
\text { LLC }\end{array}$ & June 30, 2010 \\
\hline $\begin{array}{l}\text { Norspan } \\
\text { (Australia ) }\end{array}$ & $\begin{array}{l}\text { ER patch (7-day) } \\
\text { /Transdermal }\end{array}$ & 5,10 and $20 \mathrm{mcg} / \mathrm{hr}$ & $\begin{array}{l}\text { buprenorphine base, levulinic acid, } \\
\text { oleyl oleate, povidone, Duro Tak } \\
\text { 387-2051, Duro Tak } 387-2054 \text { and } \\
\text { polyethylene terephthalate }\end{array}$ & $\begin{array}{l}\text { Mundipharma } \\
\text { and Grunenthal }\end{array}$ & April 4, 2005 \\
\hline Probuphine & $\begin{array}{l}\text { Implant (6-month) } \\
\text { /subcutaneous }\end{array}$ & NA & $\mathrm{NA}$ & $\begin{array}{l}\text { Titan } \\
\text { Pharmaceuticals, } \\
\text { Inc. }\end{array}$ & $\begin{array}{l}\text { July } 2008 \\
\text { (opioid } \\
\text { addiction, phase } \\
\text { III; chronic pain, } \\
\text { phase I) }\end{array}$ \\
\hline
\end{tabular}


The transdermal buprenorphine patch, Transtec, was first launched in Switzerland and Germany in 2001 and is now marketed all over Europe. ${ }^{4}$ It is used to treat moderate to severe cancer pain and severe pain which does not respond to nonopioid analgesics. It is not suitable for the treatment of acute pain. Butrans is a 7-day patch for the relief of moderate to severe pain in patients requiring continuous, aroundthe-clock opioid treatment for an extended period of time. It was approved by US FDA on June 30, 2010 and marked by Purdue Pharma with the strengths of 5,10 and $20 \mathrm{mcg} / \mathrm{hr}$. Norspan is also a 7-day analgesic patch but available in Austrilia. ${ }^{5}$ It is indicated for the treatment of moderate chronic pain not responding to non-opioids, dosed in 5,10 or $20 \mathrm{mcg} / \mathrm{hr}$ patches.

Probuphine ${ }^{6}$ is a novel subcutaneous implant to deliver six months of buprenorphine. Titan Pharmaceuticals announced positive, statistically significant results from its randomized, double-blind, placebo controlled, multi-center Phase III clinical trial of Probuphine for the potential treatment of opioid addiction in July 2008. The phase I study of Probuphine on treatment of chronic pain is still going on.

\subsubsection{Pharmacology of buprenorphine}

Buprenorphine is usually described as a " $\mu$-receptor partial agonist", "mixed opioid receptor agonist-antagonist", "high affinity mu agonist/kappa antagonist", or "narcotic antagonist analgesic". ${ }^{7}$ It is used clinically for pain management and the treatment of opioid dependency. The exact mechanisms of action of buprenorphine are not fully understood. Antinociceptive effect of buprenorphine is primarily mediated by the $\mu$-opioid receptor. The data shows that buprenorphine can attenuate or abolish the action of other full agonists, such as morphine, and produce a submaximal antinociceptive effect. ${ }^{8-10}$ Therefore buprenorphine is usually accepted as a "partial" $\mu$ opioid receptor agonist. ${ }^{11-15}$ It is also a $\kappa$-receptor antagonist. ${ }^{10,16}$ In addition, recent discovery shows that buprenorphine has the affinity to delta and ORL-1 receptor ${ }^{17}$ (Table 1-2).

Buprenorphine has a unique bell-shaped dose-response curve for antinociception. ${ }^{2,18-20}$ The analgesia response of buprenorphine in rat tails warm water study did not grow with increased dose. The dose for the highest response was found at 3 $\mathrm{mg} / \mathrm{kg} .{ }^{20}$ On the contrary, dose-response curve decreased with increasing dose at high dose range. The exact mechanisms are not fully understood. The possible mechanisms could be partial agonism at the $\mu$ opioid receptor and, in some cases, antagonism at the kappa or delta opioid receptor. ${ }^{17}$ Recent data ${ }^{17}$ suggest that the action of buprenorphine at the ORL-1 receptor could be responsible for the ceiling effect and for the bell-shaped dose-response curves observed after administration of the drug in the radiant heat tail flick assay. The antinociceptive effect of buprenorphine is attenuated by the ability of the drug to activate the ORL-1 receptor in opioid receptor knockout mice. 
Table 1-2. Apparent $K_{i}$ values of buprenorphine for the various members of the opioid receptor family.

\begin{tabular}{ccccc}
\hline Animals & Mu & Kappa & Delta & ORL-1 \\
\hline Rat Brain $^{21}$ & $0.08 \pm 0.02$ & $0.11 \pm 0.05$ & $0.42 \pm 0.04$ & $285 \pm 30$ \\
Monkey Brain $^{22}$ & $0.08 \pm 0.01$ & $0.44 \pm 0.08$ & $0.82 \pm 0.11$ & not determined \\
\hline
\end{tabular}

Data are presented as mean \pm SEM $(n M)$.

Source: Lutfy K, Cowan A 2004. Buprenorphine: A unique drug with complex pharmacology. Curr Neuropharmacol 2(4):395-402. Reprinted with permission. 


\subsubsection{Pharmacokinetics of buprenorphine}

\subsubsection{Pharmacokinetic parameters}

Ohtani et al. ${ }^{2,23}$ published the pharmacokinetic parameters of buprenorphine in rats by intravenous administration at doses of $0.06 \mathrm{mg} / \mathrm{kg}$ and $0.6 \mathrm{mg} / \mathrm{kg}$, and claimed that the plasma concentration of buprenorphine declined biexponentially after intravenous administration and the mean $\mathrm{t}_{1 / 2}$ (elimination half-life), CL (clearance), $\mathrm{V}_{\mathrm{ss}}$ (volume distribution at steady-state), and MRT (mean residence time) values for buprenorphine were $2.8 \mathrm{hr}, 1.4 \mathrm{~L} / \mathrm{hr} / \mathrm{kg}, 4.2 \mathrm{~L} / \mathrm{kg}$, and $3.0 \mathrm{hr}$ at the dose of $0.6 \mathrm{mg} / \mathrm{kg}$ and $2.4 \mathrm{hr}, 1.32 \mathrm{~L} / \mathrm{hr} / \mathrm{kg}, 3.5 \mathrm{~L} / \mathrm{kg}$, and $2.8 \mathrm{hr}$ at the dose of $0.06 \mathrm{mg} / \mathrm{kg}$, respectively. Gopal et al. ${ }^{24}$ reported the pharmacokinetic parameters of buprenorphine in rats after receiving a single intravenous bolus dose of $0.1,0.3,1,3,10$ or $30 \mathrm{mg} / \mathrm{kg}$. The mean $\mathrm{t}_{1 / 2}, \mathrm{CL}, \mathrm{V}_{\mathrm{ss}}$, and MRT values for buprenorphine were $4.75-5.28 \mathrm{hr}, 2.7-6.1 \mathrm{~L} / \mathrm{hr} / \mathrm{kg}, 8.37-18.2 \mathrm{~L} / \mathrm{kg}$, and 3.02-3.83 hr, respectively. Yu et al. ${ }^{25}$ indicated that the pharmacokinetic values of $\mathrm{t}_{1 / 2}$, $\mathrm{CL}$, and $\mathrm{V}_{\text {ss }}$ were $2.9 \mathrm{hr}, 4.3 \mathrm{~L} / \mathrm{hr} / \mathrm{kg}, 6.5 \mathrm{~L} / \mathrm{kg}$, respectively, after intravenous administration of the buprenorphine solution at the dose of $2.4 \mathrm{mg} / \mathrm{kg}$ in the mouse.

\subsubsection{Absorption distribution metabolism and excretion (ADME)}

Due to the extensive first-pass effect, the bioavailability of buprenorphine in humans following oral administration is approximately $10 \%{ }^{26}$ The systemic bioavailability of buprenorphine varies by various routes of administration in rats. After single doses of $0.2 \mathrm{mg} / \mathrm{kg}$ buprenorphine solution, the systemic bioavailability of buprenorphine has been studied in female rats. The mean bioavailabilities were intravenous, 98\%; intrarectal, 54\%; intrahepatoportal, 49\%; sublingual, 13\%; and intraduodenal, $9.7 \%$, relative to the $100 \%$ bioavailability from the intra arterial route. ${ }^{27}$

After an intravenous dose of $0.2 \mathrm{mg} / \mathrm{kg}^{28,29}$ buprenorphine in the rats, high levels of buprenorphine were found in the lung, heart, kidney and liver, and buprenorphine level in the brain was higher than that in the plasma. Two metabolic pathways, ${ }^{2} \mathrm{~N}$-dealkylation by CYP3A4 and glucuronidation, were well documented in rat, rabbit, dog, baboon, and rhesus monkey, ${ }^{15}$ and could lead to the three major metabolites of buprenorphine, buprenorphine-3-glucuronide; $N$-dealkylbupurenorphine (norbuprenorphine) and norbuprenorphine-3-glucuronide. The primary metabolite is buprenorphine-glucuronide and the rest are norbuprenorphine and norbuprenorphine-glucuronide. The conjugated metabolites, buprenorphine-glucuronide and norbuprenorphine-glucuronide, are mostly excreted in bile in rats. ${ }^{23}$ Independent of species, buprenorphine is mainly excreted through the bile $\mathrm{e}^{23,30}$ and eliminated in the faeces, and a small amount of metabolites are excreted through the urine by urinary excretion. ${ }^{2}$ Enterohepatic circulation of buprenorphine was found in rats, dogs, monkeys and human. ${ }^{23,30,31}$ The conjugated metabolites, buprenorphine-glucuronide and norbuprenorphine-glucuronide, in the bile could be absorbed after the return of the parent drugs, buprenorphine and norbuprenorphine, to the intestinal tract. ${ }^{2}$ 


\subsection{Injectable Analgesics for Rodents}

Current injectable analgesic for rodents can be divided into two groups: opioid and non-steroidal anti-inflammatory drug (NSAID) analgesics. The dose, administration route and formulation of analgesics for rodents are listed in Table 1-3.

\subsubsection{Opioid injectable analgesics for rodents}

Opioid analgesics are the derivatives of opiates and usually used in moderate to severe types of pain for rodents. ${ }^{32}$ These types of analgesics used in rodents can be classified as agonists, partial agonists, and agonist/antagonist. ${ }^{33}$ Agents, such as morphine, codeine, and propoxyphene, are agonists which bind to central nervous system opiate $\mu$-receptors to produce analgesia and reduce the perception of pain. Agents, such as buprenorphine, are partial agonists of $\mu$-receptor, but the maximal analgesia produced is less than full agonist. Agents, such as butorphanol and nalbuphine, are agonistantagonists. These drugs are agonist of some opioid receptors, but act as antagonist at other opioid receptors.

\subsubsection{Buprenorphine}

Buprenorphine is currently considered as the standard opioid analgesic for rats and mice. ${ }^{34}$ It is the most commonly used and reported ${ }^{35}$ analgesic for rodents. It is the partial agonist for the $\mu$-receptor and has a relatively long duration of analgesic action ( 8 to 12 hours). Buprenorphine is used as an analgesic for pain relief after surgical procedures and is considered to be approximately 30 times stronger than morphine. Buprenorphine is well absorbed after intramuscular (IM) and subcutaneous (SC) injection, and is metabolized in the liver. In rats, the drug concentrates in the liver, brain, gastrointestinal tract and placenta. It is then excreted in urine, and via breast milk. It is therefore not recommended to be given to pregnant or lactating rats. ${ }^{36}$

The commercial product of buprenorphine is Buprenex. Each $\mathrm{mL}$ of Buprenex contains $0.324 \mathrm{mg}$ buprenorphine hydrochloride (equivalent to $0.3 \mathrm{mg}$ buprenorphine), $50 \mathrm{mg}$ anhydrous dextrose, water for injection and $\mathrm{HCI}$ to adjust $\mathrm{pH}$.

\subsubsection{Morphine}

Morphine is a highly potent opiate analgesic drug which interacts predominantly with the $\mu$-opioid receptor. In clinical practice, morphine is regarded as the gold standard of analgesics used to relieve severe or agonizing pain. The primary side effect of morphine for rodents is respiratory depression. Fortunately, the effects are species specific. For example, morphine can produce analgesia without respiratory depression in the hamster. ${ }^{44}$ 
Table 1-3. Recommended analgesics for rats and mice.

\begin{tabular}{|c|c|c|c|c|c|c|}
\hline Drug & Species & Dose & $\begin{array}{c}\text { Administration } \\
\text { Route }\end{array}$ & Frequency & \multicolumn{2}{|c|}{ Formulation of Commercial Product } \\
\hline \multirow[t]{2}{*}{ Buprenorphine } & Rats & $0.01-0.1 \mathrm{mg} / \mathrm{kg}^{37-39}$ & $\mathrm{SC}^{37-39}, \mathrm{IV}^{37,38}$ & q 6-12 hr ${ }^{37-39}$ & \multicolumn{2}{|c|}{$\begin{array}{l}\text { Buprenorphine hydrochloride }(0.324 \mathrm{mg} / \mathrm{mL} \text {, equivalent to } 0.3 \\
\text { mg buprenorphine per } \mathrm{mL})\end{array}$} \\
\hline & Mice & $0.05-0.1 \mathrm{mg} / \mathrm{kg}^{37,39,40}$ & $\mathrm{SC}^{37,39,40}, \mathrm{IV}^{37}$ & $\begin{array}{l}\text { q } 12 \mathrm{hr}^{37,40} \\
\text { q } 3-6 \mathrm{hr}^{39}\end{array}$ & \multicolumn{2}{|c|}{$\begin{array}{l}\text { Anhydrous dextrose }(50 \mathrm{mg} / \mathrm{mL}) \\
\text { Water; } \\
\mathrm{HCl} \text { (adjust } \mathrm{pH})\end{array}$} \\
\hline \multirow[t]{2}{*}{ Butorphanol } & Rats & $2 \mathrm{mg} / \mathrm{kg}^{38,39}$ & $\mathrm{SC}^{38,39}$ & $\mathrm{q} 4-6 \mathrm{hr}^{38,39}$ & \multicolumn{2}{|c|}{$\begin{array}{l}\text { Butorphanol tartrate }(1 \text { or } 2 \mathrm{mg} / \mathrm{mL}) \text {; } \\
\text { Citric acid }(3.3 \mathrm{mg} / \mathrm{mL})\end{array}$} \\
\hline & Mice & $1-5 \mathrm{mg} / \mathrm{kg}^{39,40}$ & $\mathrm{SC}^{39,40}$ & $\begin{array}{l}\text { q } 6 \mathrm{hr}^{40} \\
\mathrm{q} 8-12 \mathrm{hr}^{39}\end{array}$ & \multicolumn{2}{|c|}{$\begin{array}{l}\text { Sodium citrate }(6.4 \mathrm{mg} / \mathrm{mL}) \\
\mathrm{NaCl}(6.4 \mathrm{mg} / \mathrm{mL}) \\
\text { Benzethonium chloride }(0.1 \mathrm{mg} / \mathrm{mL} \text {, in multiple dose vial only }) \text {. }\end{array}$} \\
\hline \multirow[t]{2}{*}{ Ketoprofen } & Rats & $5 \mathrm{mg} / \mathrm{kg}^{37}$ & $\mathrm{SC}^{37,41}$ & $\operatorname{sid}^{37}$ & \multirow{2}{*}{\multicolumn{2}{|c|}{$\begin{array}{ll}\text { Ketoprofen }(100 \mathrm{mg} / \mathrm{mL}) ; & \text { L-arginine }(72 \mathrm{mg}) \\
\text { Benzyl alcohol }(1 \%) ; & \text { Citric acid (adjust } \mathrm{pH})\end{array}$}} \\
\hline & Mice & $5 \mathrm{mg} / \mathrm{kg}^{37}$ & $\mathrm{SC}^{37}$ & $\operatorname{sid}^{37}$ & & \\
\hline \multirow[t]{2}{*}{ Morphine } & Rats & $\begin{array}{l}2.5-5 \mathrm{mg} / \mathrm{kg}^{39} \\
10 \mathrm{mg} / \mathrm{kg}^{42}\end{array}$ & $\mathrm{SC}^{39,42}, \mathrm{IM}^{42}$ & q 2-4 hr 39,42 & \multicolumn{2}{|c|}{$\begin{array}{l}\text { Morphine sulfate pentahydrate }(0.5 \text { or } 1 \mathrm{mg} / \mathrm{mL}) \\
\mathrm{NaCl}(9 \mathrm{mg} / \mathrm{mL})\end{array}$} \\
\hline & Mice & $\begin{array}{l}2.5 \mathrm{mg} / \mathrm{kg}^{39} \\
10 \mathrm{mg} / \mathrm{kg}^{42}\end{array}$ & $\mathrm{SC}^{39,42}, \mathrm{IM}^{42}$ & $\mathrm{q} 2-4 \mathrm{hr}^{39,42}$ & \multicolumn{2}{|c|}{$\mathrm{NaOH}$ and/or $\mathrm{HCl}$ (adjust $\mathrm{pH})$} \\
\hline \multirow[t]{2}{*}{ Carprofen } & Rats & $2.5-5 \mathrm{mg} / \mathrm{kg}^{37,39}$ & $\mathrm{SC}^{37,39}, \mathrm{IM}^{37}$ & $\operatorname{sid}^{37}$, bid $^{39}$ & \multirow{5}{*}{$\begin{array}{l}\text { Carprofen }(50.0 \mathrm{mg} / \mathrm{mL}) ; \\
\text { Arginine }(30.0 \mathrm{mg} / \mathrm{mL}) ; \\
\text { Glycocholic acid }(88.5 \mathrm{mg} / \mathrm{mL}) ; \\
\text { Benzyl alcohol }(10.0 \mathrm{mg} / \mathrm{mL}) ; \\
\text { Meloxicam }(5.0 \mathrm{mg} / \mathrm{mL}) ; \\
\text { Alcohol }(15 \%) ; \\
\text { Glycofurol }(10 \%) ; \\
\text { Poloxamer } 188(5 \%) ; \\
\mathrm{NaCl}(0.6 \%) ;\end{array}$} & \multirow{5}{*}{$\begin{array}{l}\text { Lecithin }(169.0 \mathrm{mg} / \mathrm{mL}) ; \\
\mathrm{NaOH}(6.17 \mathrm{mg} / \mathrm{mL}) ; \\
\mathrm{NaOH} \text { and/or } \mathrm{HCl} \text { (adjust } \mathrm{pH}) \\
\text { Water. } \\
\text { Glycine }(0.5 \%) ; \\
\text { Meglumine }(0.3 \%) ; \\
\text { Water; } \\
\mathrm{NaOH} \text { and } \mathrm{HCl} \text { (adjust } \mathrm{pH})\end{array}$} \\
\hline & Mice & $1-5 \mathrm{mg} / \mathrm{kg}^{37}$ & $\mathrm{SC}^{37}$ & $\operatorname{sid}^{37}$ & & \\
\hline \multirow[t]{3}{*}{ Meloxicam } & Rats & $1-2 \mathrm{mg} / \mathrm{kg}^{39,43}$ & $\mathrm{SC}^{39,43}$ & $\operatorname{sid}^{39,43}$ & & \\
\hline & & & & & & \\
\hline & Mice & $1 \mathrm{mg} / \mathrm{kg}^{39}$ & $\mathrm{SC}^{39}$ & $\operatorname{sid}^{39}$ & & \\
\hline
\end{tabular}

Q: every.

Sid: once daily.

Bid: twice daily. 
Each $\mathrm{mL}$ of commercial available morphine sulfate injection contains morphine sulfate pentahydrate $0.5 \mathrm{mg}$ or $1 \mathrm{mg}$ and sodium chloride $9 \mathrm{mg}$. It may also contain sodium hydroxide and/or hydrochloric acid for $\mathrm{pH}$ adjustment. ${ }^{45}$

\subsubsection{Butorphanol}

Butorphanol is a synthetic opiate agonist-antagonist that is structurally related to morphine. Butorphanol binds to opiate receptors at sites in the central nervous system and alters perception and response to pain. ${ }^{46}$ It is more potent than morphine, but unlike morphine it possesses less cardiovascular effects, and respiratory center sensitivity.

Butorphanol Tartrate (Torbutrol, Torbugesic, Stadol) is available as Torbutrol injectable $(0.5 \mathrm{mg} / \mathrm{mL}$ in $10 \mathrm{~mL}$ vials $)$, Torbugesic injectable $(10 \mathrm{mg} / \mathrm{mL}$ in $50 \mathrm{~mL}$ vials $)$ and Stadol injectable $(1 \mathrm{mg} / \mathrm{mL}$ and $2 \mathrm{mg} / \mathrm{mL})$. Stadol Injection is a sterile, parenteral, aqueous solution of butorphanol tartrate for intravenous or intramuscular administration. In addition to 1 or $2 \mathrm{mg}$ of butorphanol tartrate, each $\mathrm{mL}$ of solution contains $3.3 \mathrm{mg}$ of citric acid, $6.4 \mathrm{mg}$ sodium citrate, and $6.4 \mathrm{mg}$ sodium chloride, and $0.1 \mathrm{mg}$ benzethonium chloride (in multiple dose vial only) as a preservative.

\subsubsection{NSAID injectable analgesics for rodents}

Non-steroidal anti-inflammatory drugs (NSAIDs) can be classified as traditional cyclooxygenases (COX) inhibitor and COX-2 inhibitor. Traditional NSAIDs have been used more for mild pain and anti-inflammation activities than for chronic pain. ${ }^{47}$ While $2^{\text {nd }}$ generation NSAIDs can alleviate acute pain, such as that produced by surgery ${ }^{48}$ and have significant analgesic properties which overlap with the activity of the opioids. ${ }^{47}$

First generation of NSAIDs, such as Aspirin, can decrease the production of prostaglandin by inhibiting COX, which reduces pain and also inflammation. Research suggested that most of the adverse effects of NSAIDs were regulated by blocking the COX-1 enzyme, while the analgesic effects were mediated by the COX-2 enzyme. Thus the $2^{\text {nd }}$ generation COX2 inhibitors, such as carprofen and meloxicam, were developed to inhibit only the COX-2 enzyme.

NSAIDs can be administered via IM, SC or PO. The metabolism and excretion of NSAIDs vary widely among agents and species. In general, the NSAIDS are metabolized by the liver and excreted by the kidneys. ${ }^{47}$

\subsubsection{Carprofen}

Carprofen also belongs to NSAID which is a selective COX-2 inhibitor. It is used in the treatment of pain for either short term or long term use. It can also be used as an alternative to opioid based post-operative pain. Usually carprofen is used to treat post- 
operative, injury-related, and chronic (e.g., cancer-related) pain in dogs and cats as well as rabbits, coatis and many small mammals. ${ }^{49}$ Carprofen for animals is one of the most reliable and useful active principles available to veterinarians for treating animals in pain. It has a dual mode of action: $\mu$-receptor agonism and mono-amine reuptake inhibition, which produces mild anti-anxiety results. ${ }^{50}$ The advantage of this drug is less respiratory and cardiovascular side effects than the opioids, and longer acting time. Recommended dose of carprofen for rats is $5-10 \mathrm{mg} / \mathrm{kg}$ orally ${ }^{51}$ or $5-10 \mathrm{mg} / \mathrm{kg} \mathrm{SC}{ }^{52}$

The drug is metabolized in the liver and primarily eliminated in feces with a small percentage being eliminated in the urine. There is some enterohepatic recycling of the drug.

Carprofen is marketed under 75 trade names ${ }^{49}$ around the world. Carprofen is available as Tramadol hydrochloride injection $(50 \mathrm{mg} / 1 \mathrm{ml}$ and $100 \mathrm{mg} / 2 \mathrm{ml}),{ }^{53}$ which is not currently marketed in the US for human use, but it is still available in other countries. ${ }^{54}$ Each $\mathrm{mL}$ of carprofen Injectable contains $50.0 \mathrm{mg}$ carprofen, $30.0 \mathrm{mg}$ arginine, $88.5 \mathrm{mg}$ glycocholic acid, $169.0 \mathrm{mg}$ lecithin, $10.0 \mathrm{mg}$ benzyl alcohol, $6.17 \mathrm{mg}$ sodium hydroxide, with additional sodium hydroxide and hydrochloric acid as needed to adjust $\mathrm{pH}$, and water for injection. ${ }^{55}$

\subsubsection{Meloxicam}

Meloxicam is also a NSAID and shows a more selective inhibition of COX-2. It is used to treat mild to moderate to severe pain in rats. Meloxicam is relatively expensive and thus may not be practical for everyday use. A dose of $1-2 \mathrm{mg} / \mathrm{kg}$ orally or subcutaneously once a day for rats has been given. ${ }^{56}$ Both injectable and oral formulations of meloxicam have been licensed for use in dogs and injectable ones for use in cats since 1980's.

The commercial product of Meloxicam is Metacam. Each $\mathrm{mL}$ of this sterile product for injection contains meloxicam $5.0 \mathrm{mg}$, alcohol 15\%, glycofurol 10\%, poloxamer $1885 \%$, sodium chloride $0.6 \%$, glycine $0.5 \%$, and meglumine $0.3 \%$, in water for injection, $\mathrm{pH}$ adjusted with sodium hydroxide and hydrochloric acid. ${ }^{57}$

\subsubsection{Ketoprofen}

Ketoprofen is a propionic acid derivative. ${ }^{58}$ It is a NSAID which inhibits both the cyclooxygenase and lypoxygenase pathways of arachidonic acid breakdown. It is a potent, non-narcotic analgesic and anti-pyretic agent. ${ }^{59}$

Ketoprofen is available as Anafen Injection $(100 \mathrm{mg} / \mathrm{mL})$. Each $\mathrm{mL}$ contains 100 mg ketoprofen, $72 \mathrm{mg}$ L-arginine, $1 \%$ benzyl alcohol as a preservative and suitable citric acid to adjust $\mathrm{pH}^{60}$ 


\subsection{Sustained Release Analgesic Formulations in Animals}

Several sustained release analgesic formulations have been studied in animals. Transdermal fentanyl patches have been studied in cats ${ }^{61}, \operatorname{dogs}^{61,62}$, sheep $^{63}$, and rabbits ${ }^{64}$ for up to 72 hours. Liposomal formulations of opioid analgesics have been tested in mice and rats. Administration of liposomal oxymorphone at the time of surgery improved postsurgical recovery in mice. ${ }^{34}$ Liposome-encapsulated oxymorphone was found to provide prolonged relief of postsurgical visceral pain for at least $48 \mathrm{hr}$ in a rat short-gut syndrome model. ${ }^{65}$ Liposome-encapsulated oxymorphone or morphine was effective in preventing hyperalgesia in rat neuropathic pain model for up to seven days. ${ }^{66}$ Liposomeencapsulated opioid analgesic, hydromorphone, was tested in a chronic constriction injury thermal hyperalgesia rat model of neuropathic pain, and found that preemptive injection of liposome-encapsulated hydromorphone prevented hyperalgesia in this model for five days. ${ }^{67}$ However, these tested sustained release analgesic patches or liposomal formulations were not practical and economic to rodents. In 2011, commercially available buprenorphine $\mathrm{HCl}$ sustained release product was announced by ZooPharm (Fort Collins, CO). The formulation is composed of a biocompatible organic solvent and biodegradable copolymer with an average molecular weight of approximately $5500 \mathrm{Da}$ and a 50:50 molar ratio of DL-lactide to $\varepsilon$-caprolactone. It was claimed that the formulation could provide sustained release of buprenorphine for 72 hours after subcutaneous administration. ${ }^{68}$ The disadvantages of this polymeric delivery system are potential irritation and relatively expensive considering the large amount of rodents used in research.

\subsection{In Vitro Dissolution Model for Parenteral Dosage Forms}

So far there are no standard regulatory methods for parenteral sustained release products at present. ${ }^{69,70}$ Over the past decade, several methods have been attempted to describe drug release from parenteral products and the current in vitro release methods for parenterals might be divided into four broad categories (Figure 1-2): sample-andseparate (SS) ${ }^{69,70}$ continuous flow (CF) ${ }^{69,70}$ ultrafiltration, ${ }^{70}$ and dialysis techniques including rotating dialysis, ${ }^{71}$ dialysis sac, ${ }^{70}$ and microdialysis. ${ }^{70}$ The advantages and disadvantages of each method were discussed by Diane J. Burgess ${ }^{70}$ and Claus Larsen. ${ }^{69}$

Sample-and-separate method ${ }^{69}$ is a closed system and generally used to test microsphere based or gel formulations. The formulation is introduced into a vessel/vial containing the release medium. The experiment is then conducted at constant temperature with agitation. At predetermined time intervals, samples are taken from the supernatant and analyzed for released drug content. After sampling, same volume of fresh medium is added to the system to maintain a constant volume.

USP (United States Pharmacopeia) apparatus 4 is the commonly used continuous flow method ${ }^{69,72}$ for microparticulate systems. The microparticulate formulation is usually placed in the release cell and the dissolution medium is pumped into the cell at constant flow rate. The drug content can be analyzed directly from the effluent and 
Figure 1-2. Schematic illustration of different in vitro dissolution methods for parenterals.

(A) Sample and separation method (S-S).

(B1) Closed-loop and (B2) open-loop configuration for flow-through cell apparatus.

(C) Ultrafiltration technique.

(D1) Dialysis membrane-based technique (DMB).

(D2) Rotating dialysis cell model.

(D3) Reversed dialysis sac technique.

(D4) Microdialysis sampling technique.

Source: Larsen C, Larsen SW, Jensen H, Yaghmur A, Ostergaard J 2009. Role of in vitro release models in formulation development and quality control of parenteral depots. Expert Opin Drug Deliv 6(12):1283-1295. Reprinted with permission.

Source: Krishna R, Yu L 2008. Biopharmaceutics Applications in Drug Development.336-358. Reprinted with permission.

Source: Schultz K, Mollgaard B, Frokjaer S, Larsen C 1997. Rotating dialysis cell as in vitro release method for oily parenteral depot solutions. Int J Pharm 157(2):163-169. Reprinted with permission. 


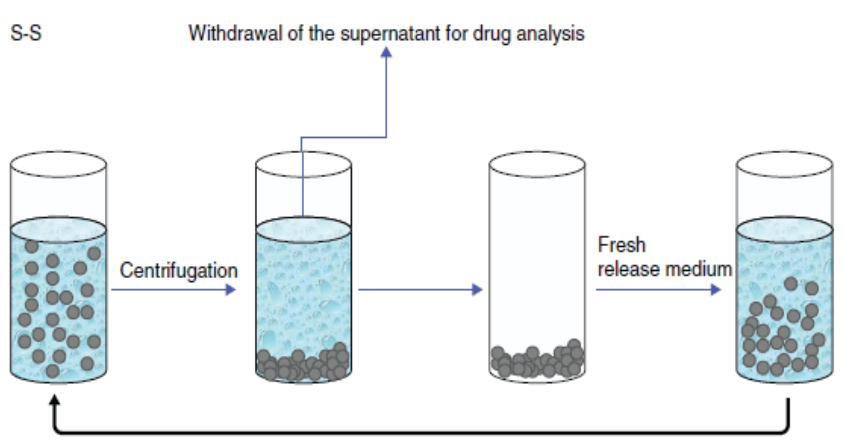

(A)

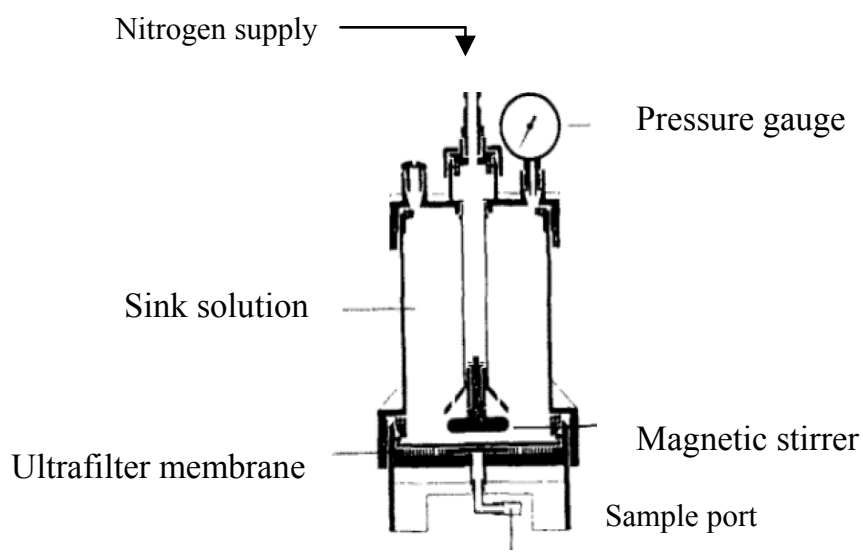

(C)

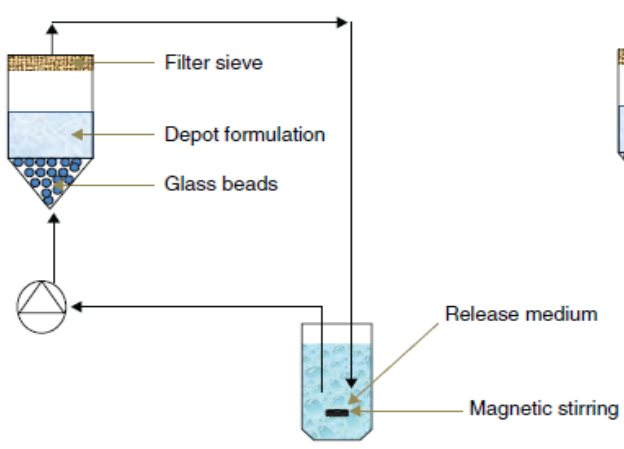

(B1)

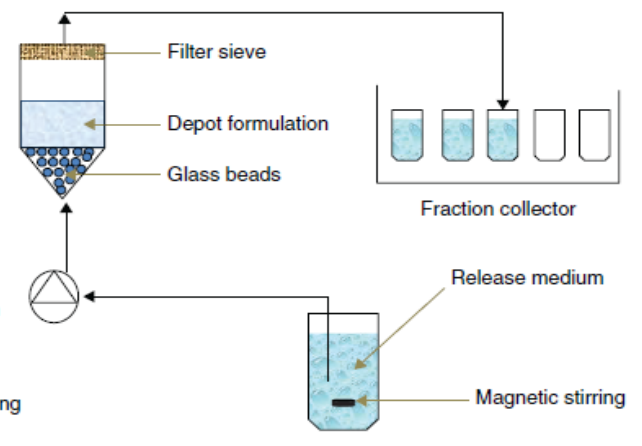

(B2)

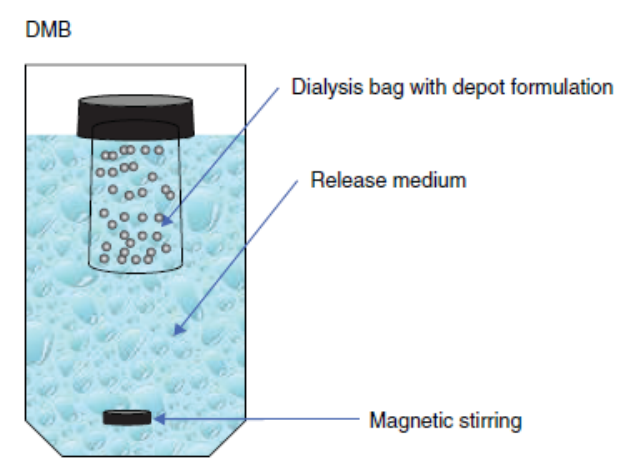

(D1) 


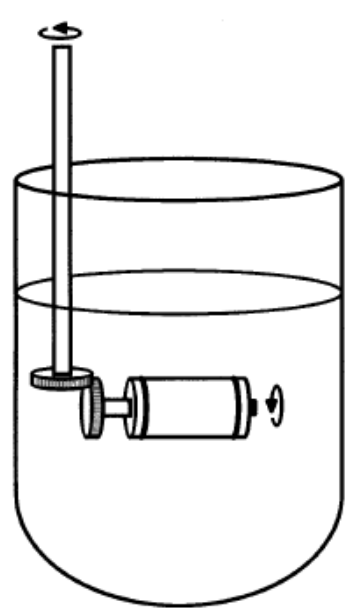

(D2)

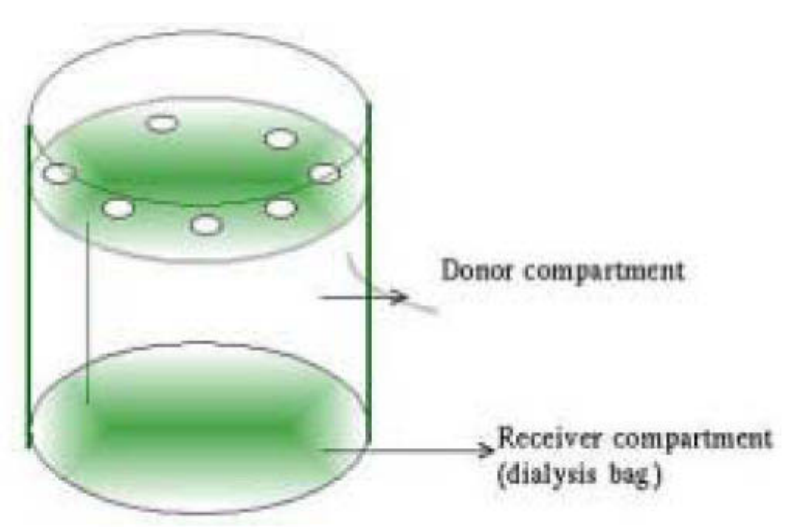

(D3)

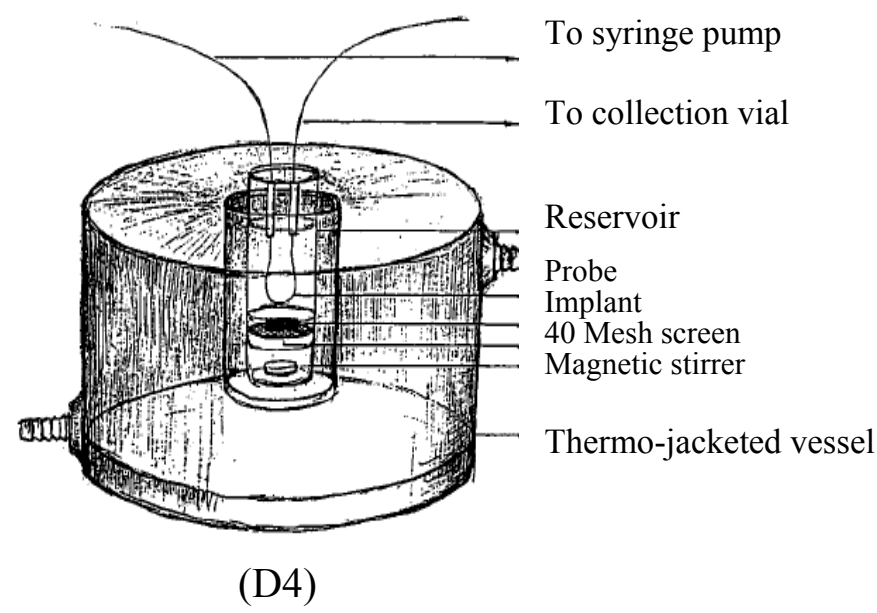

Figure 1-2. (Continued). 
medium can be recirculated into the system, or monitored after collecting the samples.

Ultrafiltration ${ }^{73}$ is a membrane filtration technique. This separation technique is usually used to purify and concentrate macromolecular solutions, especially protein solutions. Low molecular weight solutes pass through the semipermeable membrane and suspended solids and solutes of high molecular weight are retained. In order to solve the difficulty of separation of the delivery system from the media, ultrafiltration ${ }^{70,74}$ method is proposed for the evaluation of drug release profile from colloidal carriers such as submicron emulsions and nanoparticles at low pressure. It was claimed that ultrafiltration method at low pressure was versatile, sensitive and capable of distinguishing between different kinetic behaviors.

The dialysis membrane-based (DMB) method most often can be referred to as two-compartment release model. It usually consists of a small donor compartment and a large acceptor compartment separated by the dialysis membrane. Among the DMB methods, (1) rotating dialysis cell model ${ }^{71}$ is well characterized and consists of a small donor compartment (5-8 $\mathrm{mL})$ and a large acceptor compartment $(1000 \mathrm{~mL})$ separated by a dialysis membrane. Lipophilic (oily) solutions, aqueous suspensions, microspheres and liposomes have been tested in this model and the drug release mechanism has been well characterized. Also the in vitro and in vivo correlation has been established for a particular formulation in this model. (2) Float A Lyzer $^{69}$ is the commercially available dialysis membrane-based model which works with the gentle agitation in the donor compartment, resulting in lower drug transport rates. (3) In dialysis sac ${ }^{70,75}$ system, dispersed phase (e.g. emulsion) is directly placed into the large donor chamber with the stirred sink solution and the sacs contain only media. At predetermined time intervals, dialysis bags were withdrawn for drug content analysis. (4) Microdialysis technique ${ }^{76}$ usually is used for continuous measurement of free, unbound analyte concentrations in the extracellular fluid of virtually any tissue. The microdialysis probe consists of a shaft with a semipermeable hollow fiber membrane at its tip, which is connected to inlet and outlet tubing. The microdialysis system used for parenterals ${ }^{70,75}$ consists of the syringe pump, microdialysis probe, 40-mesh screen and a magnetic stirrer system. The implant was usually placed on the screen, and the microdialysis probe was placed at a fixed distance from the implant. The release medium was constantly stirred with the aid of a magnetic stirrer at the bottom of the reservoir. At predetermined time intervals, the samples were collected directly into the autosampler injection vials and drug content was further determined.

\subsection{IVIVC}

\subsubsection{Definition}

An in vitro-in vivo correlation (IVIVC) has been defined by the Food and Drug Administration (FDA) as "a predictive mathematical model describing the relationship between an in vitro property (usually the extent or rate of drug release) and a relevant in 
vivo response (e.g. plasma concentration or amount of drug absorbed)". ${ }^{77}$ The United States Pharmacopoeia (USP) also defines IVIVC as "the establishment of a relationship between a biological property, or a parameter derived from a biological property produced by a dosage form, and a physico-chemical characteristic of the same dosage form". ${ }^{78}$

\subsubsection{Categories}

According to the FDA, IVIVC are categorized as Levels A, B, C and Multiple C. The classification of correlation level is based upon the ability of predictive mathematical model to correlate the entire plasma drug concentration time curve or other important pharmacokinetic parameters with the in vitro dissolution profile or parameters. According to FDA guidance, (1) Level A correlation is "a predictive mathematical model for the relationship between the entire in vitro dissolution/release time course and the entire in vivo response time course (e.g., the time course of plasma drug concentration or amount of drug absorbed)". ${ }^{78}$ Level A correlation is general linear and represents a point-to-point relationship between in vitro dissolution and the in vivo input rate of the drug from the dosage form. The key of the correlation is point-to-point correlation and therefore the in vitro dissolution and in vivo input rate curves can share the same mathematical equation to describe the drug release behavior. (2) Level B correlation is "a predictive mathematical model for the relationship between summary parameters that characterize the in vitro and in vivo time courses (e.g., models that relate the mean in vitro dissolution time to the mean in vivo dissolution time, the mean in vitro dissolution time to the mean residence time in vivo, or the in vitro dissolution rate constant to the absorption rate constant)" ${ }^{78}$ A Level B correlation utilizes all of the in vitro and in vivo data, but it is not considered to be a point-to-point correlation since it does not reflect the entire in vivo plasma level curve. Since Level B correlation cannot uniquely reflect the actual in vivo plasma level curve, it has less application than Level A correlation. It is least useful for regulatory purposes. (3) Level $\mathrm{C}$ correlation is "a predictive mathematical model of the relationship between the amount dissolved in vitro at a particular time (or the time required for in vitro dissolution of a fixed percent of the dose, e.g., T50\%) and a summary parameter that characterizes the in vivo time course". ${ }^{78}$ Level C represents a single point correlation between one dissolution time point (e.g., $\mathrm{T}_{50} \%, \mathrm{~T}_{90} \%$ ) and one important pharmacokinetic parameter (e.g., $\mathrm{AUC}, \mathrm{C}_{\max }$ or $\mathrm{T}_{\max }$ ). Level $\mathrm{C}$ correlations can be useful in the early stages of formulation development when pilot formulations are being selected. (4) A multiple Level C correlation represents relationship between the amount of drug dissolved at several time points of the dissolution profile and one or several pharmacokinetic parameters of interest. Multiple Level C correlations can be as useful as Level A correlations. But if a multiple Level C correlation is possible, then a Level A correlation is also likely and is preferred. ${ }^{78}$

Level A correlation is the most informative and useful for waiver of an in vivo bioequivalence study (biowaiver). Level B and C IVIVCs have been applied in formulation development, optimizing manufacturing processes, quality control processes, and characterizing the release patterns of newly formulated IR and modified-release 
products relative to the references. ${ }^{79}$ A multiple Level $\mathrm{C}$ correlation may be used to justify a biowaiver by establishment of the correlation over the entire dissolution profile with one or more pharmacokinetic parameters of interest.

\subsubsection{Evaluation}

The objective of developing IVIVC is to establish a predictive mathematical model describing the relationship between an in vitro property and a relevant in vivo response. ${ }^{78}$ It is recommended by FDA that the IVIVC relationship should be demonstrated consistently with two or more formulations with different release rates to result in corresponding differences in absorption profiles. The evaluation of IVIVC should be able to demonstrate that predictability of in vivo performance of a drug product from its in vitro dissolution profile. Generally, Level A IVIVC validation can be assessed by prediction of plasma profiles or AUC from in vitro dissolution data using the developed IVIVC.

The objective of IVIVC evaluation is "to estimate the magnitude of the error in

predicting the in vivo bioavailability results from in vitro dissolution data". ${ }^{78}$ Two criteria, internal and external criteria, are required for the validation process. (1) Internal predictability is based on the initial data used to define the IVIVC model and should be studied for all IVIVCs. The criteria of internal predictability are that "average absolute percent prediction error ( $\% \mathrm{PE}$ ) of $10 \%$ or less for $\mathrm{C}_{\max }$ and $\mathrm{AUC}$ establishes the predictability of the IVIVC. In addition, the $\% \mathrm{PE}$ for each formulation should not exceed 15\%". Percent prediction error (\% PE) is calculated by Equation 1-1. (2) Evaluation of

$$
\% P E=\frac{\text { Observed value }- \text { Pr edicted value }}{\text { Observed value }} \times 100
$$

external predictability of the IVIVC should be performed as a final determination of established IVIVC to be used as a surrogate for bioequivalence if the internal predictability of the IVIVC is inconclusive. ${ }^{78}$ This evaluation is based on additional test data set that was not used in developing the IVIVC model, and the important criterion is that " $\%$ PE of $10 \%$ or less for Cmax and AUC establishes the external predictability of an IVIVC". 


\section{CHAPTER 2. DEVELOPMENT OF A SUSTAINED RELEASE BUPRENORPHINE FORMULATION FOR MAINTAINING PROLONGED ANALGESIA IN RATS AND MICE}

\subsection{Introduction}

Rodents are the most universally used laboratory animals and are often subjected to research involving painful procedures. In US, the National Association for Biomedical Research (NABR) estimated that 23 million rats and mice were needed for research in 1998 and that rodents accounted for $95 \%$ of the animals used. ${ }^{80}$ The Home Office showed that 3.6 million animals were used in scientific procedures in 2010 and $80 \%$ of them were rats and mice in the UK. ${ }^{81}$ Animal welfare regulations require that anesthetic or analgesia should be provided to the animals involved in painful procedures.

Numerous studies cite buprenorphine as a potent opioid analgesic for managing pain in rodents as it exhibits excellent analgesic activity and minimal side effects. ${ }^{12,82-87} \mathrm{~A}$ survey of current literature indicates that buprenorphine is one of the most widely used analgesics in rodents for its excellent analgesic activity and long duration of action ${ }^{68,88-90}$ and has been extensively used in both laboratory and companion animals for almost three decades. ${ }^{90}$ Buprenorphine has a complex pharmacological profile. It is generally accepted that buprenorphine is $\mu$-opioid receptor partial agonist, $\kappa$-opioid receptor antagonist, and $\delta$-receptor agonist. ${ }^{87}$ It has a broad analgesic profile, relative wide safety margin ${ }^{91}$ and offers the opportunity to treat different pain conditions for rodents. ${ }^{92}$ Elizabeth A. Tolley et al demonstrated that buprenorphine has a analgesia duration of 6 to $8 \mathrm{hr}$ in rats $(0.5$ $\mathrm{mg} / \mathrm{kg})$ and 3 to $5 \mathrm{hr}$ in mice $(2.0 \mathrm{mg} / \mathrm{kg})$ after subcutaneous injection buprenorphine solution using conventional hot-plate and tail-flick assays. ${ }^{93}$ But in order to maintain effective analgesia levels, buprenorphine must be administered at least 2 or 3 times daily for each animal. Frequent dosing requires more personnel effort and more handling to the animals. However, no commercial sustained release analgesic for rodents was available when we started the project in 2008. In 2011, Patricia L Foley ${ }^{68}$ published a biodegradable polymer delivery system composited of buprenorphine hydrochloride, a biocompatible organic solvent, and DL-lactide- $\varepsilon$-caprolactone copolymer with an average molecular weight of approximately $5500 \mathrm{Da}$. It was claimed that this product could provide a 48 to 72 hours period of analgesia for rats. But skin irritation is potentially a problem for this product. ${ }^{68}$ The other disadvantage of this product is that the cost of biodegradable polymer delivery system is much higher than the hydrophobic based solution system.

Parenteral long-acting lipophilic solutions have been in clinical use for more than three decades. ${ }^{94}$ Compared with other parenteral delivery system, the composition is relatively simple and has the attributes ${ }^{94}$ of uncomplicated manufacture and feasible longterm stability. Some citric acid esters, such as acetyl triethyl citrate (ATEC), acetyl tributyl citrate (ATBC), acetyl trihexyl citrate, and acetyl trioctyl citrate, are safe to use in cosmetics. ${ }^{95}$ Both ATEC and ATBC were nonirritating to the skin in rabbits and human clinical studies. ${ }^{95}$ These indicated the potential to apply citric acid esters as nonirritating 
and low cost vehicles in drug delivery. In order to reduce the stress of frequent handling and injection as well as improve the welfare of research animals, this study developed a long-acting sustained release hydrophobic formulation of buprenorphine which is capable of maintaining analgesia in rats and mice for 3 to 5 days following a single subcutaneous administration.

\subsection{Materials and Methods}

\subsubsection{Materials}

Buprenorphine base was obtained from Diosynth Inc. (Morrisville, NC). Triethyl citrate (TEC), acetyl triethyl citrate (ATEC), tributyl citrate (TBC) and acetyltributyl citrate (ATBC) were obtained from Vertellus Specialties Inc. (Greensboro, NC). Polyethylene glycol 400 (PEG 400) was obtained from Dow Chemicals, Inc. (Danbury, CT). Soybean oil and corn oil were obtained from Croda, Inc. (Columbus Circle Edison, NJ). Ethyl benzoate and benzyl benzoate were purchased from Sigma Aldrich (Atlanta, GA).

\subsubsection{Animals}

Male Sprague Dawley rats (approximately $300 \mathrm{~g}$ ) and mice (approximately $30 \mathrm{~g}$ ) were obtained from Charles River Laboratories (Wilmington, MA). The animals were group-housed in polycarbonate caging with ad libitum access to food and water. The experiments were conducted according to protocols approved by the Institutional Animal Care and Use Committee (IACUC).

\subsubsection{Analytical methodology for in vitro and in vivo studies}

\subsubsection{Analytical methodology for in vitro studies}

Two different HPLC methods were developed to quantitate buprenorphine in samples obtained from in vitro studies. Method 1 was used for equilibrium solubility and dissolution studies. Method 2 was used for stability studies. A silica-gel column was used in Method 2 rather than C-18 column in Method 1 was because multiple injections of buprenorphine samples containing highly lipophilic excipients resulted in contamination of $\mathrm{C}-18$ column and low sensitivity of the assay.

\subsection{HPLC method 1 for equilibrium solubility and dissolution studies}

The mobile phase used in method 1 was composed of a mixture of $51 \mathrm{mM}$ $\mathrm{KH}_{2} \mathrm{PO}_{4}$ buffer ( $\mathrm{pH} 5.35$ ) containing $0.0675 \%(\mathrm{v} / \mathrm{v})$ of $2 \mathrm{~N} \mathrm{NaOH}$ and acetonitrile (45:55, 
v/v). A discovery C-18 column (2.1 x $100 \mathrm{~mm}$ ) (Supelco, Bellefonte, PA) was used. The oven temperature was $40^{\circ} \mathrm{C}$ and the flow rate was $0.3 \mathrm{~mL} / \mathrm{min}$. The total run time was 6 minutes. Buprenorphine was detected with a florescence detector at $\lambda_{\text {Exitation }}=292 \mathrm{~nm}$ and $\lambda_{\text {Emission }}=350 \mathrm{~nm}$.

\subsection{HPLC method 2 for stability studies}

Waters Silica-gel column (4.6x150 mm) (Milford, MA) was used for this method. The mobile flow rate was $1.0 \mathrm{mg} / \mathrm{min}$. A mixture of $50 \mathrm{mM}$ sodium acetate $(\mathrm{pH}$ was adjusted to 3.75 with acetic acid) and acetonitrile $(20: 80, \mathrm{v} / \mathrm{v})$ was used as the mobile phase. Total run time was 7 minutes. Buprenorphine was detected with florescence detector at $\lambda_{\text {Exitation }}=292 \mathrm{~nm}$ and $\lambda_{\text {Emission }}=350 \mathrm{~nm}$.

\subsection{Preparation of standard solutions}

The primary standard stock solution $(1.68 \mathrm{mg} / \mathrm{mL})$ for the validation study of Method 1 was prepared by accurately weighing $16.8 \mathrm{mg}$ of buprenorphine, dissolving it with acetonitrile and diluting the resulting solution to $10 \mathrm{~mL}$ with acetonitrile. The secondary standard working solutions $(0.038,0.076,0.38,7.6$ and $38 \mu \mathrm{g} / \mathrm{mL})$ were prepared by serially diluting the primary standard stock solution with $50 \% \mathrm{ACN}$. To test intra-day accuracy, a set of standard samples were injected 3 times on the same day. To test inter-day accuracy, three sets of standard samples were freshly prepared individually every day and then injected once every day.

The primary standard stock solution $(1.17 \mathrm{mg} / \mathrm{mL})$ for the validation study of Method 2 was prepared by accurately weighing $11.7 \mathrm{mg}$ of buprenorphine, dissolving it with acetonitrile and diluting to $10 \mathrm{~mL}$ with additional acetonitrile. The secondary standard working solutions $(0.03,0.12,0.29,1.17$ and $5.85 \mu \mathrm{g} / \mathrm{mL})$ were prepared by serially diluting the primary standard stock solution with $50 \%$ ACN. To test intra-day accuracy, a set of standard samples were injected 5 times on the same day. To test interday accuracy, three sets of standard samples were freshly prepared individually every day and then injected once every day.

\subsubsection{Analytical methodology for in vivo studies}

Validated LC/MS/MS methods were developed for quantifying the drug concentrations in plasma samples obtained from in vivo studies.

\subsection{Equipment}

The HPLC system consisted of two Shimadzu LC-10ADvp pumps (Shimadzu Scientific Instruments Inc., Columbia, MD), a Shimadzu SIL-HTc autosampler, and a 
HILIC silica column $(5 \mu \mathrm{m}, 150 \times 4.6 \mathrm{~mm})$ with a guard column $(10 \times 2.1 \mathrm{~mm})($ Waters Inc., Milford, MA). Harvard syringe pump (Harvard Apparatus, South Natick, MA). API 4000 tandem triple quadrupole mass spectrometer (Applied Biosystems, Foster City, CA) and Source SF 5000 LC/MS Gas Generator (Parker Hannifin, Haverhill, MA) were also used. Analyst ${ }^{\circledR}$ software (Applied Biosystems, Version 1.4.1, Foster City, CA) was used for data acquisition and processing.

\subsection{HPLC conditions}

A gradient method was used to supply eluent to the mass spectrometer. The mobile phase consisted of solutions A and B. Solution A consisted of ACN : water : formic acid (4.95:95:0.05, v/v) containing $2 \mathrm{mM}$ ammonium formate. Solution B consisted of ACN : water : formic acid (95:4.95:0.05, v/v) containing $2 \mathrm{mM}$ ammonium formate. The time program of the gradient method was such that it started with $90 \%$ of Solution B and $10 \%$ of Solution A. The concentration of Solution B was then decreased linearly from $90 \%$ to $40 \%$ within 3.5 minutes and then immediately increased to $90 \%$ and maintained at $90 \%$ for 2 minutes. The concentration of Solution B was then decreased linearly from $90 \%$ to $40 \%$ within 0.5 minute and then linearly increased to $90 \%$ within 0.5 minute and maintained at $90 \%$ for 4 minutes. The total run time was 10 minutes. The column was operated at room temperature and the flow-rate of the mobile phase was 0.5 $\mathrm{mL} / \mathrm{min}$.

\subsection{Switch valve}

The eluent from the HPLC system was only introduced directly into the mass spectrometer between running time of $4.6 \mathrm{~min}$ and $5.6 \mathrm{~min}$. The eluent from the HPLC system within the first 4.6 minutes and after 5.6 minutes was introduced directly into the waste collector.

\subsection{MS conditions}

The MS was set in the positive ion mode. The ion-spray voltage was $5.0 \mathrm{kV}$ and desolvation temperature was $600^{\circ} \mathrm{C}$. Ion source gas one, ion source gas two, curtain gas and collision gas flows were set at 55, 60,14, and 6 of the instrument units, respectively. The mass analyzer was set at multiple-reaction-monitoring (MRM) mode with a dwell time of $300 \mathrm{~ms}$ for each ion pair. Ion transition of 468.3 to 468.3 was selected for buprenorphine detection and 472.3 to 472.3 for buprenorphine-D4 detection. The compound dependent parameters for buprenorphine and buprenorphine-D4 were as follows: declustering potential (DP) of 47, entrance potential (EP) of 10, collision energy (CE) of 50, and collision cell exit potential (CXP) of 13. Resolution of the mass analyzer was set at unit resolution. Optimization of the mass spectrometric conditions were carried out by infusing $50 \mathrm{ng} / \mathrm{mL}$ of analyte solution dissolved in a mixture of acetonitrile and water $(50 / 50, \mathrm{v} / \mathrm{v})$ at $10 \mu \mathrm{L} / \mathrm{min}$ using a syringe pump. 


\subsection{Process of plasma samples}

The internal standard solution $(50 \mathrm{ng} / \mathrm{mL})$ of buprenorphine-D4 was prepared by diluting buprenorphine-D4 standard solution $(100 \mu \mathrm{g} / \mathrm{mL})$ with $0.1 \%$ formic acid solution. Twenty-five microliters of rat plasma was spiked with $25 \mu \mathrm{L}$ of BuprenorphineD4 internal standard solution and $25 \mu \mathrm{L}$ of $50 \% \mathrm{ACN}$. The resulting mixture was mixed well by vortexing for 10 seconds and centrifuged at $14 \mathrm{~K}$ rpm for 1 minute, and then deproteinated by adding $25 \mu \mathrm{L}$ of $15 \%$ trichloroacetic acid solution. The mixture was then vortexed for one minute and kept in $-20^{\circ} \mathrm{C}$ for 30 minutes. The samples were then centrifuged for 10 minutes at $14 \mathrm{k} \mathrm{rpm}$ using an Eppendorf 5415C centrifuge (Brinkman Instruments Incorporated, Westbury, NY). The supernatant was transferred to HPLC sample vials and the drug content of the samples was determined by the above mentioned validated LC/MS/MS assay. The ratio of peak area of buprenorphine to that of peak area of buprenorphine-D4 was used to determine the buprenorphine plasma concentration.

\subsection{Preparation of calibration standard samples and quality control samples}

The primary standard stock solution $(500 \mu \mathrm{g} / \mathrm{mL})$ for buprenorphine calibration standard samples was prepared by accurately weighing approximately $25 \mathrm{mg}$ of buprenorphine and dissolving it in $10 \mathrm{~mL}$ volumetric flask with ACN. The secondary standard working stock solutions with the following concentrations - $0.4,2,10,50,100$, 150,200 , and $250 \mathrm{ng} / \mathrm{mL}$ were prepared by serially diluting the primary standard stock solution with $50 \% \mathrm{ACN}$. The calibration standard samples were prepared as follows: twenty-five microliters of rat plasma was spiked with $25 \mu \mathrm{L}$ of Buprenorphine-D4 internal standard solution and $25 \mu \mathrm{L}$ of secondary standard working stock solutions. The resulting mixture was mixed well by vortexing for 10 seconds and centrifuged at $14 \mathrm{~K}$ $\mathrm{rpm}$ for one minute, and then deproteinated by adding $25 \mu \mathrm{L}$ of $15 \%$ trichloroacetic acid solution. The mixture was then vortexed for one minute and kept at $-20^{\circ} \mathrm{C}$ for 30 minutes. The samples were then centrifuged for 10 minutes at $14 \mathrm{k} \mathrm{rpm}$ using a centrifuge. The supernatant was transferred to HPLC sample vials and the drug content of the samples was determined by a validated LC/MS/MS assay.

Four standard samples $(2,50,100,200 \mathrm{ng} / \mathrm{mL})$ were injected three times in the same day to test the intra-day accuracy assay. Two more sets of the standard samples (2, $50,100,200 \mathrm{ng} / \mathrm{mL}$ ) were freshly prepared in different day and analyzed to test the interday variation. The primary quality control (QC) stock solution $(500 \mu \mathrm{g} / \mathrm{mL})$ was independently prepared by weighing approximately $25 \mathrm{mg}$ of buprenorphine powder and dissolving it with ACN. The QC working solutions $(2,50,100$, and $200 \mathrm{ng} / \mathrm{mL})$ were prepared by serially diluting the primary QC stock solution with $50 \% \mathrm{ACN}$ solution. 


\subsubsection{In vitro studies}

\subsubsection{Determination of equilibrium solubility of buprenorphine}

Buprenorphine base powder (approximately $300 \mathrm{mg}$ ) was suspended in $1.0 \mathrm{~mL}$ of solvents (TEC, ATEC, TBC, ATBC, Ethyl benzoate, benzyl benzoate, PEG 400, soybean oil and corn oil) in $2.0 \mathrm{~mL}$ Eppendorf centrifuge tubes. After vortexing, the resulting suspensions were then shaken at $100 \mathrm{rpm}$ in a shaking incubator maintained at $25^{\circ} \mathrm{C}$ and $37^{\circ} \mathrm{C}$. After 1,2 and 5 days, the suspensions were vortexed again and then centrifuged at $12 \mathrm{~K} \mathrm{rpm}$ for 5 minutes. Approximately $100 \mu \mathrm{L}$ of the clear supernatant was carefully removed and the buprenorphine samples were diluted with acetonitrile. One milliliter of this solution was further diluted with acetonitrile-water mixture $(50: 50, \mathrm{v} / \mathrm{v})$ and analyzed using a validated HPLC method.

\subsubsection{Determination of stability of buprenorphine in various solvents}

Approximately $200 \mu \mathrm{L}$ of saturated or nearly saturated buprenorphine solutions in different solvents (TEC, ATEC, TBC, ATBC, Ethyl benzoate, benzyl benzoate, PEG 400, soybean oil and corn oil) were diluted to $10 \mathrm{~mL}$ by adding the respective solvents. After filtration, the resulting solutions were stored at $25^{\circ} \mathrm{C}$ and $40^{\circ} \mathrm{C}$. At $0,7,14,21$ and 28 day, $100 \mu \mathrm{L}$ of the solutions were removed from the containers and diluted to $10 \mathrm{~mL}$ in volumetric flasks with acetonitrile. The resulting diluted solution was then analyzed for drug content using a validated HPLC method.

\subsubsection{In vitro drug release study}

Six solvents were selected for in vitro formulation screening test based on the results of aforementioned studies. An appropriate amount of buprenorphine base powder was accurately weighed and added to an appropriate amount of solvent in $20 \mathrm{~mL}$ glass scintillation vials. The subsequent suspension was sonicated in a water bath maintained less than $25^{\circ} \mathrm{C}$ until all drug powder was dissolved. The drug content was verified by HPLC method. The formulation composition was listed in Table 2-1 and the buprenorphine concentration is $1.5 \%(\mathrm{w} / \mathrm{w})$.

Approximately $210 \mathrm{mg}$ of buprenorphine solution was weighed and carefully transferred into the cavity of a Teflon cylinder (internal diameter of the cavity was 15.0 $\mathrm{mm}$, internal depth was $4.1 \mathrm{~mm}$, height of the cylinder was $12.3 \mathrm{~mm}$, and the outside diameter of the cylinder was $20 \mathrm{~mm}$ ). The solution-loaded Teflon was then covered with a 40-mesh USP basket and centered at the bottom of 1L dissolution vessel. Approximately $900 \mathrm{~mL}$ of 10\% isopropyl alcohol (IPA)-40\% water-49.95\% phosphate buffer saline solution (PBS) (v/v/v) dissolution medium ( $\mathrm{pH} 7.4)$ containing $0.05 \%$ SDS was carefully transferred into each vessel at the rate of $7 \mathrm{~mL} / \mathrm{sec}$ using modified Hanson 
Table 2-1. Buprenorphine sustained release formulations for in vitro dissolution studies.

\begin{tabular}{ccc}
\hline Formulation & Solvent & $\begin{array}{c}\text { Concentration of Buprenorphine in Solvent } \\
(\mathbf{w} / \mathbf{w})\end{array}$ \\
\hline 1 & TEC & $1.5 \%$ \\
2 & ATEC & $1.5 \%$ \\
3 & TBC & $1.5 \%$ \\
4 & ATBC & $1.5 \%$ \\
5 & Benzyl Benzoate & $1.5 \%$ \\
6 & Ethyl Benzoate & $1.5 \%$ \\
\hline
\end{tabular}


Media Mate (Hanson Research Corp.). The ion strength calculated by Debye-Hückel equation was adjusted by sodium chloride and kept at 0.13 . The new designed apparatus was used and the paddle speed was set at $100 \mathrm{rpm}$. The details of the apparatus will be discussed in Section 3.3.6.1. The vessels were covered with a lid and tightened with laboratory para-film in order to avoid evaporation of the medium.

The in vitro drug release studies were conducted in triplicate. At 0, $30 \mathrm{~min}$, and 1, 2, 4, 6, 9, 12, 24, 36, 48, 60, 72, 84, 96 and 120 hours. Approximately $1 \mathrm{~mL}$ dissolution medium was carefully withdrawn by Hanson Autosampler (Hanson Research Corp.) and replaced with $1 \mathrm{~mL}$ pre-warmed fresh dissolution medium. The dissolution samples were filtered through $0.45 \mu \mathrm{m}$ PVDF membranes and analyzed for drug content using the validated HPLC Method.

\subsubsection{In vivo studies}

\subsubsection{Measurement of analgesia in rats and mice using the tail flick method}

The tail flick method is classic method to evaluate the pain of analgesic. The tailflick test apparatus (Model 33, IITC Life Science, Woodland Hills, CA) was used to measure analgesia in animals following the administration of the buprenorphine solutions. In the tail flick test, the animal tail was exposed to a hot-light beam. When the pain threshold was reached, the animal flicked the tail away from the heat beam. The time is recorded and the analgesic effect at each time point was reported as the Percentage of Maximum Possible Effect (\% MPE). This method compares the latency at each time point with the animal's own average baseline latency. $\% \mathrm{MPE}$ was calculated using Equation 2-1.

$$
\% \mathrm{MPE}=\frac{\text { Latency }- \text { Baseline Latency }}{\text { Cutoff Time }- \text { Baseline Latency }} \times 100 \%
$$

where latency is the response time for the animal to flick the tail away from the hot light beam; the cutoff time was set at 10 seconds to prevent any possible tissue damage; and the baseline latency for each animal was obtained 24 hours prior to the administration of the formulations and reported as the average value of three measurements. The intensity of light beam was set at the conditions under which the baseline latency of a normal untreated rat was approximately 3 to 4 seconds.

\subsubsection{PK and PD studies of buprenorphine in rats after an intravenous injection of buprenorphine solution}

Six male Sprague Dawley rats with implanted vascular access ports (VAP) were used in this study. Before blood samples were withdrawn, the pharmacodynamic activity was evaluated via aforementioned tail flick method. Rats were then anesthetized lightly 
with isoflurane, and $0.05 \mathrm{mg} / \mathrm{kg}$ of aqueous solution of buprenorphine hydrochloride was injected intravenously via saphenous vein over $10 \mathrm{sec}$. Blood samples $(0.2 \mathrm{~mL})$ were withdrawn through the VAP at $0,5,15$, and $30 \mathrm{~min}$, and 1, 2, 3, 6, 12 and $24 \mathrm{hr}$ after administration, and collected in heparinized vial. Plasma was separated from whole blood by centrifuging the blood samples at $14 \mathrm{k}$ rpm for $7 \mathrm{~min}$ and stored at $-20^{\circ} \mathrm{C}$ until analyzed for drug content. Plasma samples were processed and then analyzed using the validated $\mathrm{LC} / \mathrm{MS} / \mathrm{MS}$ method.

\subsubsection{PK and PD studies of buprenorphine in rats after a subcutaneous injection of buprenorphine sustained release formulations}

An appropriate amount of buprenorphine base powder was accurately weighed and added to an appropriate amount of TBC in $20 \mathrm{~mL}$ glass scintillation vial (Tables 2-2 and 2-3). The subsequent suspension was sonicated periodically in a water-bath maintained less than $25^{\circ} \mathrm{C}$ until all drug powder was dissolved. The final solution was obtained by filtering the above solution through a $0.22 \mu \mathrm{m}$ syringe filter into $20 \mathrm{~mL}$ presterilized glass vial in a sterile hood and the drug concentration was verified by HPLC method.

Table 2-2 lists all the investigated buprenorphine formulations in rat studies. The tested solvents were selected based on the results of the solubility and stability studies. During the animal experiment, buprenorphine solutions $(\sim 0.2 \mathrm{~mL})$ were administered subcutaneously into rats. The syringes were weighed before and after subcutaneous administration of the solutions, and the exact weight of the buprenorphine solution administered to each rat was noted.

Before blood samples were withdrawn, the pharmacodynamic activity was evaluated via aforementioned tail flick method. Blood samples $(\sim 0.2 \mathrm{~mL})$ were then withdrawn from the saphenous vein at predetermined time points and kept in heparinized Microvette tubings (Microvette CB 300 LH, Sarstedt, Nümbrecht, Germany). The collected blood samples were immediately centrifuged at $14 \mathrm{~K}$ rpm for $7 \mathrm{~min}$ and plasma samples were stored at $-20^{\circ} \mathrm{C}$ until further analysis. The plasma samples were processed and analyzed for drug content using the validated LC/MS/MS method. The plasma concentration at each time point was reported as average \pm SD (standard deviation). At 120 hours (day 5) after subcutaneous administration of the buprenorphine solutions, the rats were anesthetized by overdosing with isoflurane, followed by pneumothorax euthanasia. The injection sites on the carcasses were carefully dissected and the injection site of the subcutaneous tissue was exposed, inspected for any residues or for any gross inflammation, and photographed. 
Table 2-2. Buprenorphine sustained release formulations tested in rats.

\begin{tabular}{cccc}
\hline Formulation & Solvent & $\begin{array}{c}\text { Concentration of Buprenorphine } \\
(\mathbf{m g} / \mathbf{g})\end{array}$ & $\begin{array}{c}\text { Dose } \\
(\mathbf{m g} / \mathbf{k g})\end{array}$ \\
\hline R1 & TBC & 3.08 & 1.5 \\
R2 & TBC & 5 & 2.25 \\
R3 & TBC & 8.5 & 5 \\
\hline
\end{tabular}

Table 2-3. Buprenorphine sustained release formulations tested in mice.

\begin{tabular}{cccc}
\hline Formulation & Solvent & $\begin{array}{c}\text { Concentration of Buprenorphine } \\
(\mathbf{m g} / \mathbf{g})\end{array}$ & $\begin{array}{c}\text { Dose } \\
(\mathbf{m g} / \mathbf{k g})\end{array}$ \\
\hline M1 & TBC & 3.9 & 8.9 \\
M2 & TBC & 0.87 & 2.2 \\
M3 & TBC & 0.44 & 1.1 \\
\hline
\end{tabular}




\subsubsection{PK and PD studies of buprenorphine in mice after subcutaneous injection of buprenorphine sustained release formulations}

Based on the results from rat studies, three buprenorphine formulations (Table 2-3) were selected, prepared and tested in mice. Approximately $50 \mu \mathrm{L}$ of buprenorphine solutions were administered subcutaneously in mice using 25 -gauge needles. The syringes were weighed before and after subcutaneous administration of the solutions, and the exact weight of the buprenorphine solution administered to each mouse was noted.

At predetermined time points after the administration, the analgesic activity of each formulation was evaluated via tail flick method in five mice and the average analgesic effect at each time point was reported as the Percentage of Maximum Possible Effect (\%MPE). Immediately after the PD study, blood samples $(0.6 \sim 1 \mathrm{~mL})$ were collected by cardiac puncture method and placed in $1.5 \mathrm{~mL}$ heparinized centrifuge tubes. Then the mice were sacrificed by cervical dislocation under isoflurane anesthesia. The injection sites of the subcutaneous tissue were carefully dissected, exposed, inspected for any gross inflammation, and photographed for any residues. The collected blood samples were immediately centrifuged at $14 \mathrm{~K} \mathrm{rpm}$ for $15 \mathrm{~min}$ and plasma samples were stored at $20^{\circ} \mathrm{C}$ until further analysis for drug content using the validated LC/MS/MS method. The average plasma concentration at each time point was reported.

\subsubsection{Toxicity evaluation of citric acid esters in rats}

Approximately $200 \mu \mathrm{L}$ of each of the sterilized citric acid esters (TEC, ATEC, TBC and ATBC) or normal saline were subcutaneously administered to four male SD rats. The animals were monitored for signs of toxicity and were weighed daily. Their eating and drinking habits were also closely monitored.

\subsection{Results and Discussion}

\subsubsection{Validation of analytical methodology}

\subsubsection{Validation of HPLC assay for in vitro studies}

The standard curves of Method 1 and Method 2 are listed.

- The standard curve of method 1 was: Peak area $=405278 * \mathrm{C}(\mu \mathrm{g} / \mathrm{mL})$

$+29.27(\mathrm{R}=0.9997)$

- The standard curve of method 2 was: Peak area $=114685.7 * \mathrm{C}(\mu \mathrm{g} / \mathrm{mL})$ - $289.9(\mathrm{R}=0.9998)$ 
Tables 2-4 and 2-5 show the intra- and inter-day variations of Methods 1 and 2 respectively. The results show that both methods were reproducible and accurate. The samples obtained from solubility studies and dissolution studies were analyzed using HPLC Method 1. A narrow and sharp buprenorphine peak (retention time was $4.30 \mathrm{~min}$ ) was obtained with the $\mathrm{C}-18$ column and the number of theoretical plates $(\mathrm{N})$ for buprenorphine was around 2,200. The Lower Limit of Detection (LLOD) of the assay for buprenorphine was $20 \mathrm{ng} / \mathrm{mL}$. The LLOQ of the assay was $42 \mathrm{ng} / \mathrm{mL}$. The samples from stability studies were analyzed using HPLC Method 2. A narrow and sharp buprenorphine peak (retention time was $3.8 \mathrm{~min}$ ) was obtained with the silica-gel column and the column efficiency for buprenorphine $(\mathrm{N})$ was approximately 6,000. The Lower Limit of Quantification (LLOD) of the assay was $12 \mathrm{ng} / \mathrm{mL}$. The LLOQ of the assay was $29 \mathrm{ng} / \mathrm{mL}$. There was no interference between the buprenorphine and solvents peaks.

\subsubsection{Validation of LC-MS/MS assay for in vivo studies}

No significant interference was observed from the endogenous compounds at the retention times of the analytes using the optimized LC-MS/MS conditions. Moreover, the chromatographic retention times of both buprenorphine and buprenorphine-D4 were 5.1 min and showed symmetrical peak shapes. The standard curve of buprenorphine was linear (Equation 2-2, $\mathrm{R}=0.9998)$ in the entire range of the standard solutions $(0.4$ $\mathrm{ng} / \mathrm{mL}$ to $250 \mathrm{ng} / \mathrm{mL})$ :

$$
\frac{\mathrm{AUC}_{\text {buprenorphine }}}{\mathrm{AUC}_{\text {buprenorphine-D4 }}}=0.01135+0.02024 \times \mathrm{C}_{\text {buprenorphine }}
$$

where $\mathrm{AUC}_{\text {buprenorphine }}$ and $\mathrm{AUC}_{\text {buprenorphine-D4 }}$ are peak area of buprenorphine and buprenorphine-D4, respectively, and $\mathrm{C}_{\text {buprenorphine }}$ is the concentration of buprenorphine.

The LLOD of the assay for buprenorphine was $0.01 \mathrm{ng} / \mathrm{mL}$ and the LLOQ of the assay for buprenorphine was $0.4 \mathrm{ng} / \mathrm{mL}$. The intra-day and inter-day variations of the assay developed for the in vivo studies are shown in Table 2-6. The results showed that the developed assay was reproducible and accurate.

\subsubsection{In vitro studies}

In order to test the effect of the hydrophobic vehicle with the varying hydrophilicity/lipophilicity on the drug release, nine vehicles were tested in solubility and stability studies. PEG 400, TEC, ethyl benzoate, ATEC, benzyl benzoate, TBC, ATBC, soybean oil and corn oil have different hydrophilicity and lipophilicity, and have been applied in pharmaceutical products. PEG $400^{96}$ is member of polyethylene glycol family. It is a clear, colorless or slightly yellow-colored, viscous liquid. It is widely used in a variety of pharmaceutical products, such as parenteral, topical, ophthalmic, oral and rectal preparations. Intraperitoneal LD50 of PEG 400 is $10.0 \mathrm{~g} / \mathrm{kg}$ for mouse and $9.7 \mathrm{~g} / \mathrm{kg}$ 
Table 2-4. Intra-day $(n=3)$ and inter-day $(n=3)$ variation of the assay 1 .

\begin{tabular}{ccc}
\hline $\begin{array}{c}\text { Buprenorphine Concentration } \\
(\boldsymbol{\mu g} / \mathbf{m L} \mathbf{)}\end{array}$ & $\begin{array}{c}\text { Intra-day RSD } \\
(\mathbf{\%})\end{array}$ & $\begin{array}{c}\text { Inter-day RSD } \\
\mathbf{( \% )}\end{array}$ \\
\hline 0.038 & 5.82 & 3.4 \\
0.076 & 3.52 & 9.3 \\
0.38 & 0.41 & 6.4 \\
7.6 & 0.51 & 6.2 \\
38 & 0.53 & 4.6 \\
\hline
\end{tabular}

Table 2-5. Intra-day $(n=5)$ and inter-day $(n=3)$ variation of the assay 2 .

\begin{tabular}{ccc}
\hline $\begin{array}{c}\text { Buprenorphine Concentration } \\
(\boldsymbol{\mu} \mathbf{g} / \mathbf{m} \mathbf{L})\end{array}$ & $\begin{array}{c}\text { Intra-day RSD } \\
(\mathbf{\%})\end{array}$ & $\begin{array}{c}\text { Inter-day RSD } \\
(\mathbf{\%})\end{array}$ \\
\hline 0.03 & 15.0 & NA \\
0.12 & 4.7 & 4.8 \\
0.29 & 3.1 & 4.5 \\
1.17 & 1.4 & 2.1 \\
5.85 & 5.0 & 1.0 \\
\hline
\end{tabular}

Table 2-6. Intra-day and Inter-day variation of the LC-MS/MS assay (n=3).

\begin{tabular}{ccc}
\hline $\begin{array}{c}\text { Buprenorphine Concentration } \\
(\mathbf{n g} / \mathbf{m} \mathbf{L})\end{array}$ & $\begin{array}{c}\text { Intra-day RSD } \\
\mathbf{( \% )}\end{array}$ & $\begin{array}{c}\text { Inter-day RSD } \\
(\mathbf{\%})\end{array}$ \\
\hline 0.3654 & 12.8 & 14.0 \\
3.654 & 1.8 & 3.2 \\
49.54 & 0.5 & 4.0 \\
104.4 & 0.2 & 3.0 \\
195.75 & 0.4 & 2.5 \\
\hline
\end{tabular}


for rat. Intravenous LD50 is $9.7 \mathrm{~g} / \mathrm{kg}$ for rat. The cLogP value is - 4.8 and it is hydrophilic solvent. Triethyl citrate (TEC) ${ }^{96}$ is a clear, viscous, odorless, and practically colorless, hygroscopic liquid with cLogP value of 1.45. It has been used as plasticizer in film coating. It has favorable safety profile and good stability. TEC has been accepted as a direct food additive by the U. S. Food and Drug Administration. LD50 is $5.9 \mathrm{~g} / \mathrm{kg}$ in rats after subcutaneous administration and $1.75 \mathrm{~g} / \mathrm{kg}$ in mice after intraperitoneal administration. Ethyl benzoate is a colorless liquid with cLogP values of 2.73 . It is a component of some artificial fruit flavors. Oral LD50 for rat is $2.1 \mathrm{~g} / \mathrm{kg}$. Acetyl triethyl citrate (ATEC) ${ }^{96}$ is a clear, odorless and colorless oily liquid with cLogP value of 3.73. It is generally regarded as a nontoxic and nonirritating material. Oral LD50 in rats is 7.0 $\mathrm{g} / \mathrm{kg}$. Intraperitoneal LDso in mouse is $1.15 \mathrm{~g} / \mathrm{kg}$. FDA has approved its pharmaceutical use as a plasticizing agent in coating. Benzyl benzoate ${ }^{96}$ is a clear, colorless, oily liquid with a slightly aromatic odor with cLogP value of 3.97. It is used as a solubilizing agent, plasticizer and nonaqueous solvent in intramuscular injections at concentrations of $0.01-$ $46.0 \%(\mathrm{v} / \mathrm{v})$ in pharmaceutical products. It is also used as a solvent and fixative for flavors and perfumes in cosmetics and food products. Besides, it is as a topical therapeutic agent in the treatment of scabies and therapeutically as a parasiticide in veterinary medicine. Oral LD50 is $1.4 \mathrm{~g} / \mathrm{kg}$ for mouse and $0.5 \mathrm{~g} / \mathrm{kg}$ for rat. Tributyl citrate (TBC) ${ }^{96}$ is a clear, odorless, practically colorless, oily liquid with cLogP value of 4.68. It is considered as chemically inert and safe material. FDA has approved its pharmaceutical use as a plasticizing agent in coatings. Oral LD50 in rats is more than 30 $\mathrm{mL} / \mathrm{kg}$. Intraperitoneal LD50 in mouse is $2.9 \mathrm{~g} / \mathrm{kg}$. Acetyl tributyl citrate (ATBC) ${ }^{96}$ is a clear, odorless, practically colorless, oily liquid with cLogP value of 6.92. It is used in oral, topical pharmaceutical formulations and films intended for direct food contact. It is generally regarded as a relatively nontoxic and nonirritating material. Intraperitoneal LD50 for mouse is more than $4 \mathrm{~g} / \mathrm{kg}$ and oral LD50 for rat is more than $31.5 \mathrm{~g} / \mathrm{kg}$. The skin irritation testing in rabbits showed that neither ATEC nor ATBC induced skin irritation. ${ }^{95}$ Soybean oil ${ }^{96}$ is a clear, pale-yellow colored, odorless or almost odorless liquid with a bland taste. It is used in cosmetics, pharmaceutical products and as bath additive to treat dry skin. In pharmaceutical products, it is used in parenteral nutrition vehicles for the oral and intravenous administration of drugs, and has been used in drug delivery systems such as liposomes, microspheres, emulsions, self-emulsifying systems, emulsions, nanoemulsions, and nanocapsules, solid-in-oil suspensions, and multiple emulsions. The intravenous LD50 is $22.1 \mathrm{~g} / \mathrm{kg}$ for mouse and $16.5 \mathrm{~g} / \mathrm{kg}$ for rat.

\subsubsection{Equilibrium solubility studies}

Table 2-7 lists the solubility of buprenorphine base in different solvents. It is apparent from the table that the drug has the highest solubility in ethyl benzoate, and lowest in corn oil and soybean oil at both 25 and $37^{\circ} \mathrm{C}$. The estimated minimum solubility was approximately $10 \mathrm{mg} / \mathrm{mL}$ calculated from the equation $\mathrm{R}_{0}=\mathrm{C}_{\mathrm{ss}} \cdot \mathrm{CL}$, where the $\mathrm{R}_{0}$ is infusion rate, $\mathrm{C}_{\mathrm{ss}}$ is plasma concentration at steady state, and CL is clearance. The clearance was from following PK study after intravenous injection of buprenorphine saline solution. The $\mathrm{C}_{\mathrm{ss}}$ was obtained from the study after subcutaneous of buprenorphine 
Table 2-7. Solubility of buprenorphine in different vehicles.

\begin{tabular}{|c|c|c|c|c|c|c|}
\hline \multirow{2}{*}{$\begin{array}{l}\text { Solvent } \\
(\mathrm{mg} / \mathrm{mL})\end{array}$} & \multicolumn{3}{|c|}{ (a) $25^{\circ} \mathrm{C}$} & \multicolumn{3}{|c|}{ a $37^{\circ} \mathrm{C}$} \\
\hline & Day 1 & Day 2 & Day 5 & Day 1 & Day 2 & Day 5 \\
\hline $\mathrm{TBC}$ & $27.2 \pm 1.7$ & $28.5 \pm 0.2$ & $28.0 \pm 0.3$ & $30.2 \pm 0.2$ & $30.3 \pm 0.6$ & $29.9 \pm 0.7$ \\
\hline ATBC & $18.9 \pm 0.8$ & $20.7 \pm 1.6$ & $20.0 \pm 1.0$ & $20.1 \pm 0.8$ & $22.5 \pm 0.5$ & $23.0 \pm 1.1$ \\
\hline TEC & $19.2 \pm 0.3$ & $18.6 \pm 0.3$ & $19.1 \pm 0.9$ & $20.7 \pm 0.1$ & $22.6 \pm 0.8$ & $21.3 \pm 1.1$ \\
\hline ATEC & $14.7 \pm 0.2$ & $14.9 \pm 0.1$ & $14.1 \pm 0.6$ & $16.2 \pm 0.3$ & $17.3 \pm 0.4$ & $17.3 \pm 0.7$ \\
\hline Ethyl benzoate & $95.7 \pm 0.2$ & $95.9 \pm 1.4$ & $95.8 \pm 1.0$ & $102.7 \pm 1.0$ & $113.8 \pm 1.3$ & $112.2 \pm 1.7$ \\
\hline Benzyl benzoate & $47.5 \pm 1.0$ & $47.0 \pm 0.3$ & $47.5 \pm 1.3$ & $48.3 \pm 0.5$ & $50.1 \pm 0.3$ & $49.4 \pm 1.4$ \\
\hline PEG 400 & $20.2 \pm 0.6$ & $21.6 \pm 0.2$ & $20.6 \pm 0.8$ & $23.4 \pm 0.7$ & $24.9 \pm 0.4$ & $24.6 \pm 0.4$ \\
\hline Soybean oil & $10.1 \pm 0.2$ & $11.0 \pm 0.1$ & $10.4 \pm 0.1$ & $11.0 \pm 0.3$ & $10.6 \pm 0.9$ & $11.6 \pm 0.6$ \\
\hline Corn oil & $10.1 \pm 0.7$ & $11.3 \pm 0.4$ & $10.5 \pm 0.3$ & $11.0 \pm 0.2$ & $11.2 \pm 02$ & $10.8 \pm 0.1$ \\
\hline
\end{tabular}

Data are presented as mean $\pm \mathrm{SEM}(\mathrm{mg} / \mathrm{mL})$.

$\mathrm{n}=3$. 
saline solution in rats. The results of calculation showed that the solubility of all the tested vehicles was more than $10 \mathrm{mg} / \mathrm{mL}$ and met the requirement of minimum solubility.

\subsubsection{Stability studies}

Figure 2-1 and Table 2-8 show the short-term stability of buprenorphine base in different solvents. It is apparent from the table that buprenorphine base was stable in all the solvents at $25^{\circ} \mathrm{C}$ for at least 30 days, but was not stable in PEG 400, soybean oil, corn oil and ATEC at $40^{\circ} \mathrm{C}$ for one month. Therefore, TEC, TBC, ATBC, ethyl benzoate, benzyl benzoate were selected for in vitro dissolution study.

\subsubsection{Dissolution studies}

Buprenorphine in the oil vehicles of TEC, ATEC, TBC, ATBC, ethyl benzoate, benzyl benzoate were selected for in vitro dissolution study. Buprenorphine in ATEC did not show the promising stability for one month, but it is one of the citric acid esters with moderate hydrophobicity and has the potential be used with other vehicle(s) in the combination formulation. Therefore, the in vitro release profile of buprenorphine in ATEC was also tested considering it was stable for 1 week at $40^{\circ} \mathrm{C}$. Figure 2-2 shows the effect of different vehicles on drug release. The release rate did not follow the order of cLogP values, but it was evident from the figure that fastest drug release was observed from formulation prepared with most hydrophilic vehicle, TEC, and 57.2\% drug release was detected at $120 \mathrm{hr}$. It was apparent from the figure that slowest drug release could be achieved from the formulations prepared with lipophilic vehicles, benzyl benzoate and TBC. Considering the LD50 of benzyl benzoate in rats $(0.5 \mathrm{~g} / \mathrm{kg}$, oral $)$ and mice $(1.4$ $\mathrm{g} / \mathrm{kg}$, oral), TBC showed the larger safety margin of LD50 in rats $(>30 \mathrm{~mL} / \mathrm{kg}$, oral $)$ and mice $(2.9 \mathrm{~g} / \mathrm{kg}$, IP) and was selected as the vehicles for in vivo study.

\subsubsection{In vivo studies}

\subsubsection{PK and PD studies in rats after intravenous injection of buprenorphine saline solution}

Figure 2-3a shows the analgesia measured by the tail flick test in rats after an i.v. administration of buprenorphine hydrochloride solution at the dose of $0.05 \mathrm{mg} / \mathrm{kg}$. At 5 minutes after the i.v. injection, 100\% MPE was obtained. Then the analgesia decreased to 50\% MPE within 9 hours, and no analgesia was observed after 24 hours after drug administration. The plasma concentration of buprenorphine versus time profile after i.v. injection in rats at a dose of $0.05 \mathrm{mg} / \mathrm{kg}$ is depicted in Figure 2-3b. Buprenorphine plasma concentrations of $14.5 \mathrm{ng} / \mathrm{mL}$ were achieved within 5 minutes after the i.v. injection. The concentration then rapidly decreased within the first 15 minutes, and then declined more slowly thereafter. The concentration of buprenorphine in plasma was less 


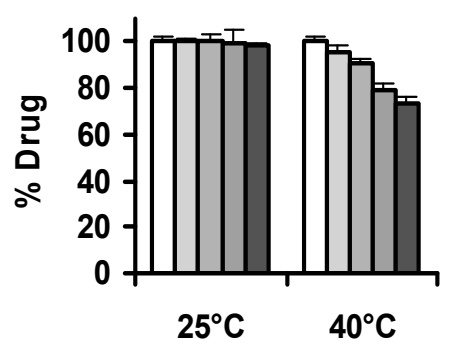

Super-Refined PEG400
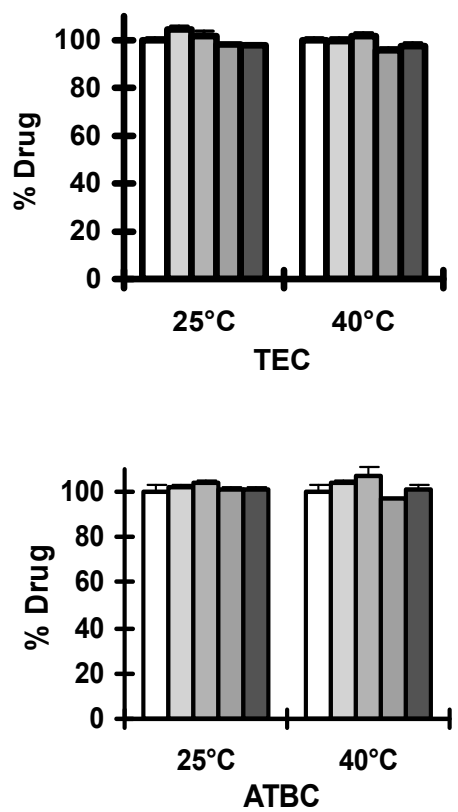
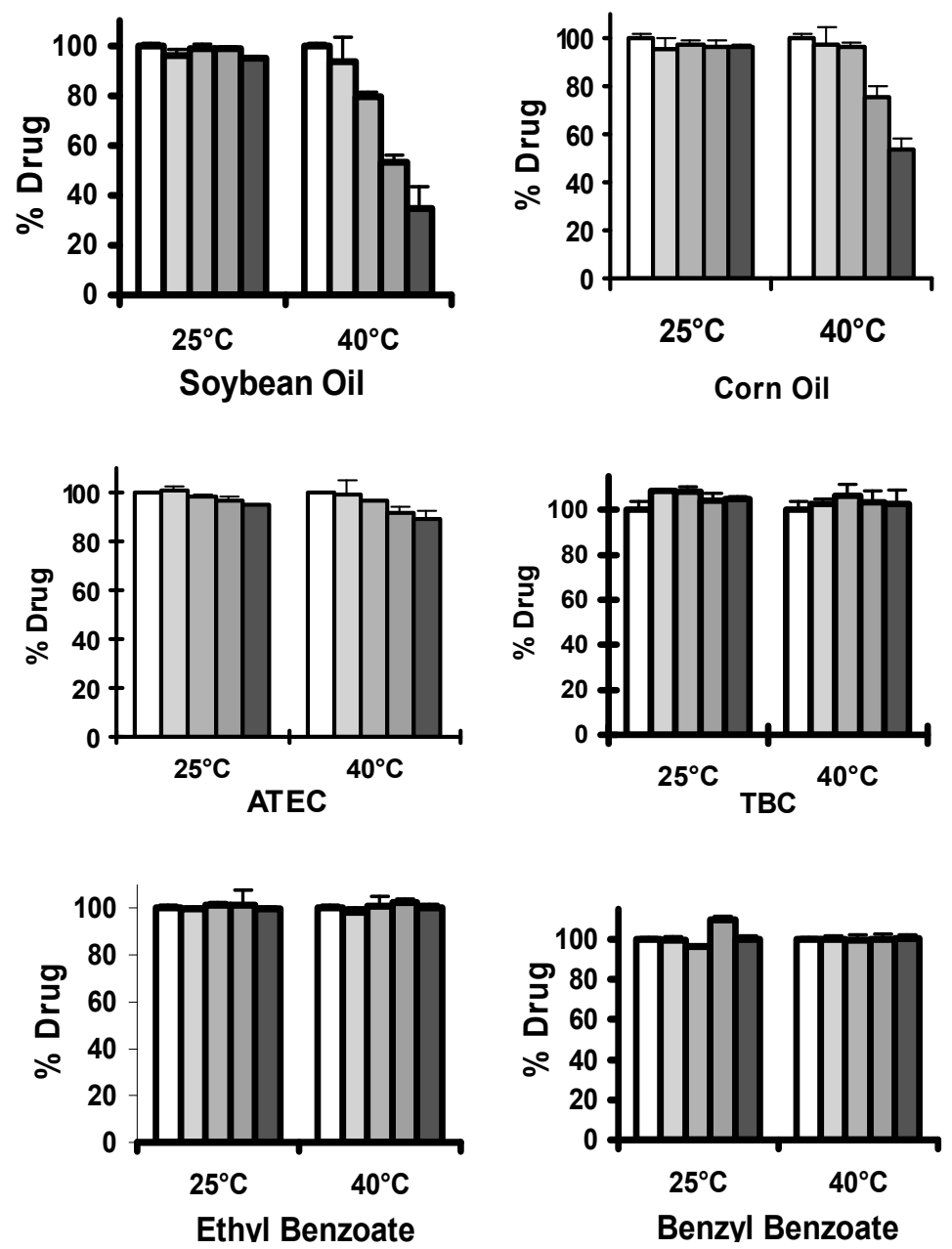

Figure 2-1. Stability of buprenorphine in various solvents. 
Table 2-8. Stability of buprenorphine base in different solvents.

\begin{tabular}{|c|c|c|c|}
\hline \multirow{2}{*}{ Solvent } & \multirow{2}{*}{$\begin{array}{c}\text { Time } \\
\text { (Week) }\end{array}$} & \multicolumn{2}{|c|}{ \% Drug Remaining } \\
\hline & & $25^{\circ} \mathrm{C}$ & $40^{\circ} \mathrm{C}$ \\
\hline \multirow[t]{5}{*}{ PEG 400} & 0 & $100.0 \pm 2.7$ & $100.0 \pm 2.7$ \\
\hline & 1 & $100.6 \pm 1.1$ & $95.8 \pm 2.3$ \\
\hline & 2 & $100.0 \pm 3.3$ & $90.6 \pm 2.5$ \\
\hline & 3 & $99.1 \pm 5.6$ & $79.0 \pm 2.6$ \\
\hline & 4 & $98.8 \pm 1.0$ & $73.8 \pm 2.2$ \\
\hline \multirow[t]{5}{*}{ TEC } & 0 & $100.0 \pm 1.0$ & $100.0 \pm 1.0$ \\
\hline & 1 & $104.5 \pm 1.2$ & $99.4 \pm 1.6$ \\
\hline & 2 & $101.7 \pm 2.0$ & $101.8 \pm 1.2$ \\
\hline & 3 & $98.1 \pm 0.3$ & $95.8 \pm 1.0$ \\
\hline & 4 & $97.7 \pm 0.7$ & $97.4 \pm 1.4$ \\
\hline \multirow[t]{5}{*}{ ATEC } & 0 & $100.0 \pm 0.1$ & $100.0 \pm 0.1$ \\
\hline & 1 & $100.9 \pm 1.6$ & $99.5 \pm 5.4$ \\
\hline & 2 & $98.2 \pm 1.0$ & $96.4 \pm 0.4$ \\
\hline & 3 & $96.8 \pm 1.5$ & $91.6 \pm 2.8$ \\
\hline & 4 & $94.9 \pm 0.5$ & $88.8 \pm 3.8$ \\
\hline \multirow[t]{5}{*}{$\mathrm{TBC}$} & 0 & $100.0 \pm 3.8$ & $100.0 \pm 3.8$ \\
\hline & 1 & $108.6 \pm 0.5$ & $102.8 \pm 1.9$ \\
\hline & 2 & $108.1 \pm 2.3$ & $106.5 \pm 4.9$ \\
\hline & 3 & $104.1 \pm 3.4$ & $103.3 \pm 5.1$ \\
\hline & 4 & $104.9 \pm 1.1$ & $102.8 \pm 6.1$ \\
\hline \multirow[t]{5}{*}{ ATBC } & 0 & $100.0 \pm 2.9$ & $100.0 \pm 2.9$ \\
\hline & 1 & $102.5 \pm 0.4$ & $103.7 \pm 1.0$ \\
\hline & 2 & $103.9 \pm 1.1$ & $107.2 \pm 3.5$ \\
\hline & 3 & $101.0 \pm 1.1$ & $96.9 \pm 0.4$ \\
\hline & 4 & $101.3 \pm 0.3$ & $101.3 \pm 1.4$ \\
\hline \multirow[t]{5}{*}{ Soybean oil } & 0 & $100.0 \pm 1.0$ & $100.0 \pm 1.0$ \\
\hline & 1 & $96.1 \pm 2.2$ & $93.7 \pm 9.8$ \\
\hline & 2 & $98.7 \pm 2.0$ & $79.4 \pm 1.7$ \\
\hline & 3 & $98.8 \pm 0.6$ & $53.1 \pm 2.9$ \\
\hline & 4 & $94.8 \pm 0.7$ & $34.8 \pm 8.6$ \\
\hline \multirow[t]{5}{*}{ Corn oil } & 0 & $100.0 \pm 2.1$ & $100.0 \pm 2.1$ \\
\hline & 1 & $95.5 \pm 4.6$ & $97.5 \pm 6.9$ \\
\hline & 2 & $97.2 \pm 1.8$ & $96.7 \pm 1.8$ \\
\hline & 3 & $96.8 \pm 2.6$ & $75.9 \pm 4.4$ \\
\hline & 4 & $96.4 \pm 1.0$ & $53.8 \pm 4.5$ \\
\hline
\end{tabular}


Table 2-8. (Continued).

\begin{tabular}{ccrr}
\hline \multirow{2}{*}{ Solvent } & Time & \multicolumn{2}{c}{ \% Drug Remaining } \\
\cline { 3 - 4 } (Week) & \multicolumn{1}{c}{$\mathbf{2 5}^{\circ} \mathbf{C}$} & $\mathbf{4 0}^{\circ} \mathbf{C}$ \\
\hline Ethyl Benzoate & 0 & $100.0 \pm 1.3$ & $100.0 \pm 1.3$ \\
& 1 & $99.9 \pm 0.7$ & $98.3 \pm 1.8$ \\
& 2 & $101.3 \pm 1.2$ & $100.8 \pm 4.3$ \\
Benzyl Benzoate & 3 & $101.1 \pm 6.6$ & $102.5 \pm 1.4$ \\
& 4 & $99.6 \pm 0.8$ & $100.2 \pm 1.5$ \\
& 1 & $100.0 \pm 0.9$ & $100.0 \pm 0.9$ \\
& 2 & $99.6 \pm 1.5$ & $99.8 \pm 1.9$ \\
& 3 & $96.3 \pm 0.6$ & $99.7 \pm 2.4$ \\
& 4 & $103.8 \pm 1.5$ & $99.8 \pm 2.9$ \\
\hline
\end{tabular}

Data are presented as mean $\pm \mathrm{SD}$. $\mathrm{n}=3$. 


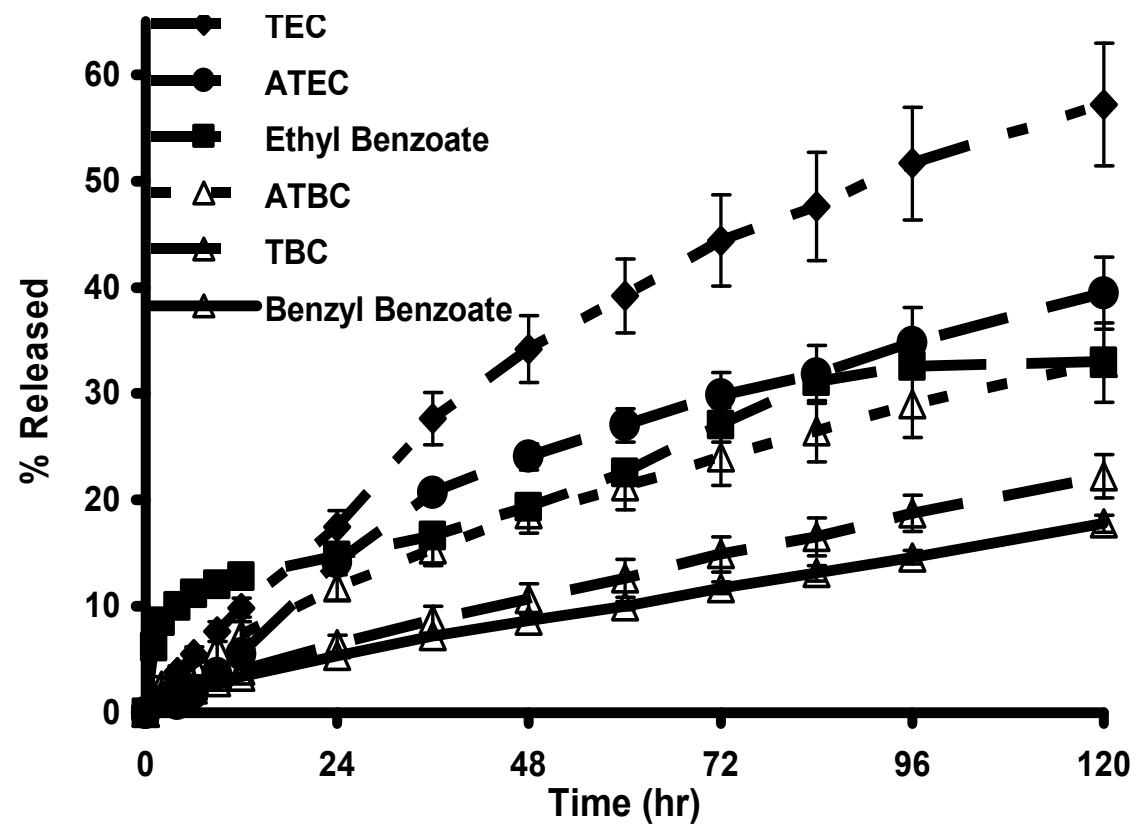

Figure 2-2. In vitro release profile of buprenorphine from oily formulations prepared with various hydrophobic vehicles.

Each data point represents an average of three measurements.

Standard deviation of three measurements is presented as error bars.

Buprenorphine concentrations in the different oily solvents were $1.5 \%$. 


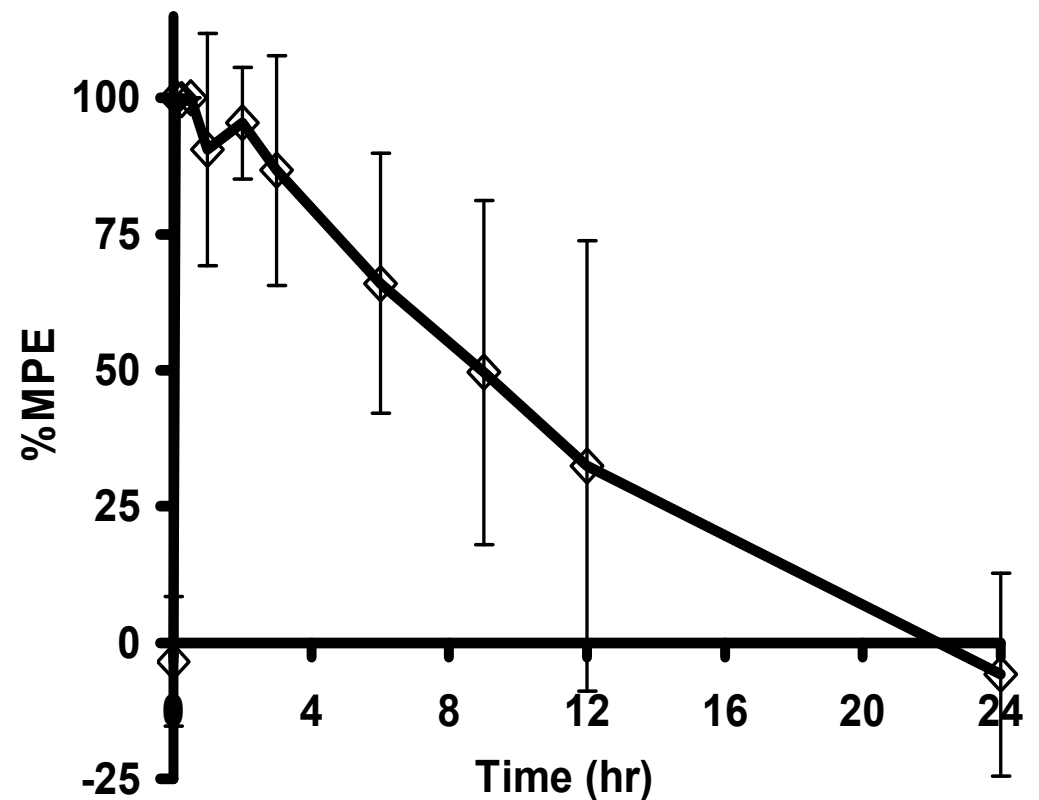

(a)

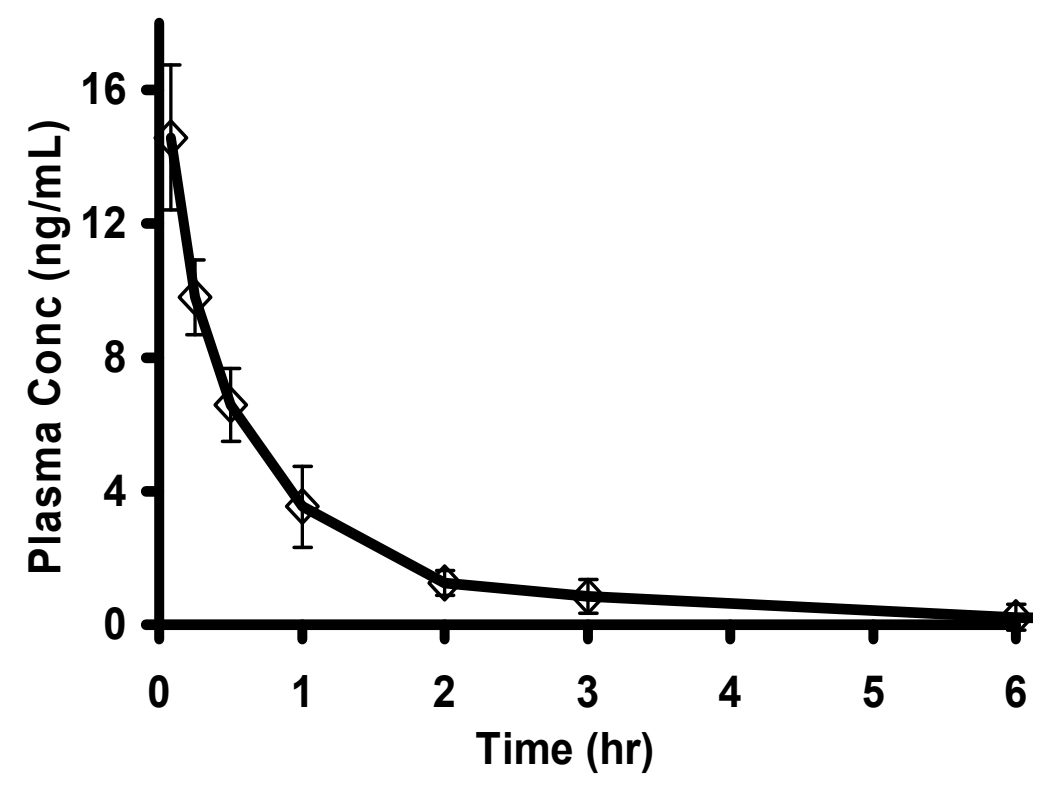

(b)

Figure 2-3. (a) Analgesic effect and (b) plasma concentration of buprenorphine in rats after a single intravenous injection of buprenorphine hydrochloride solution at the dose of $0.05 \mathrm{mg} / \mathrm{kg}$.

Each data point represents an average of six measurements.

Standard deviation of six measurements is presented as error bars.

Intensity of the tail flick tester during the experiment is $60 \%$. 
than the LLOQ of the assay after $6 \mathrm{hr}$ after the i.v. injection. The PK parameters was calculated and listed in Table 2-9.

\subsubsection{PK and PD studies in rats after subcutaneous injection of buprenorphine sustained release formulations}

Figure 2-4b shows the effect of different doses $(1.5 \mathrm{mg} / \mathrm{kg}, 2.25 \mathrm{mg} / \mathrm{kg}$ and 5 $\mathrm{mg} / \mathrm{kg}$ ) on buprenorphine plasma concentrations in rats after a single subcutaneous injection of formulations prepared with TBC. It is apparent from the figure that drug release occurred in a controlled manner from the buprenorphine solution and buprenorphine plasma concentrations increased proportionately as the dose of the drug was increased from 1.5 to 2.25 to $5 \mathrm{mg} / \mathrm{kg}$. At the dose of $5 \mathrm{mg} / \mathrm{kg}$, buprenorphine solution was able to achieve average Cmax of $22 \mathrm{ng} / \mathrm{mL}$ at 12 hours and maintain the average plasma concentrations above $7.8 \mathrm{ng} / \mathrm{mL}$ for 5 days. The average Cmax was 12 $\mathrm{ng} / \mathrm{mL}$ at 9 hours in rats that received buprenorphine solution at the dose of $2.25 \mathrm{mg} / \mathrm{kg}$ dose. The solution was able to maintain the average plasma concentration above 3.4 $\mathrm{ng} / \mathrm{mL}$ for 120 hours (5 days). Meanwhile, the average Cmax was $3.4 \mathrm{ng} / \mathrm{mL}$ at 6 hours in the rats administered with buprenorphine solution at the dose of $1.5 \mathrm{mg} / \mathrm{kg}$ dose. The solution was able to maintain the average plasma concentration above $2.3 \mathrm{ng} / \mathrm{mL}$ for 120 hours (5 days).

The pharmacology profile of buprenorphine has the character of bell-shaped ${ }^{12,13,87}$ dose-response curve for the antinociceptive action. The exact reason is not clarified and the possible reasons could be 2-receptor model and noncompetitive autoinhibition. ${ }^{16,87,97}$

The peak of the dose-response curve was found to be occurred at a dose of $3 \mathrm{mg} / \mathrm{kg}$ in the rat tail dip test using water at $45^{\circ} \mathrm{C}$ or $55^{\circ} \mathrm{C}$ as the noxious stimulus. ${ }^{7}$ Figure 2-4a indicates that the best analgesia is achieved at the dose of $1.5 \mathrm{mg} / \mathrm{kg}$. More than $55.1 \%$ MPE analgesia was maintained for 72 hours after administration. Then the analgesia declined to $20.1 \%$ MPE at 121 hours. The results shows that maintenance of higher plasma concentrations may not be necessary for achieving and maintaining better analgesia for a longer prolonged period of time.

\subsubsection{In vivo evaluation of buprenorphine sustained release formulations in mice}

Figure 2-5 shows the effect of varying buprenorphine doses $(1.1 \mathrm{mg} / \mathrm{kg}, 2.2$ $\mathrm{mg} / \mathrm{kg}, 8.9 \mathrm{mg} / \mathrm{kg}$ ) on analgesia and buprenorphine plasma concentrations in mice after a single subcutaneous injection of formulations prepared with TBC (Table 2-3). It is apparent from the Figure 2-5a that there is no significant difference in the maintenance of analgesia for up to 48 hours after injecting either 8.9 or $2.2 \mathrm{mg} / \mathrm{kg}$ dose, and greater than $52.7 \%$ analgesia was maintained for 48 hours after administering either doses of buprenorphine. Then the analgesia declined to 49\% MPE at 72 hours (3 days) and 31\% MPE at 120 hours (5 days) after injecting $8.9 \mathrm{mg} / \mathrm{kg}$ dose. After injecting $2.2 \mathrm{mg} / \mathrm{kg}$ dose, the analgesia declined to 30\% MPE at 72 hours (3 days) and 17\% MPE at 120 hours (5 days). Meanwhile, approximately 98\% MPE - initial high analgesia was achieved at 3 
Table 2-9. PK parameters of buprenorphine in rats after a single intravenous injection at the dose of $0.05 \mathrm{mg} / \mathrm{kg}$.

\begin{tabular}{ccc}
\hline $\begin{array}{c}\mathbf{t}_{\mathbf{0 . 5}} \\
(\mathbf{h r})\end{array}$ & $\begin{array}{c}\text { Vss } \\
(\mathbf{L} / \mathbf{k g})\end{array}$ & $\begin{array}{c}\mathbf{C l} \\
(\mathbf{L} / \mathbf{h r} / \mathbf{k g})\end{array}$ \\
\hline $0.59 \pm 0.11$ & $3.60 \pm 0.63$ & $4.89 \pm 0.67$ \\
\hline
\end{tabular}

Data are presented as mean $\pm \mathrm{SD}$. $\mathrm{n}=6$. 


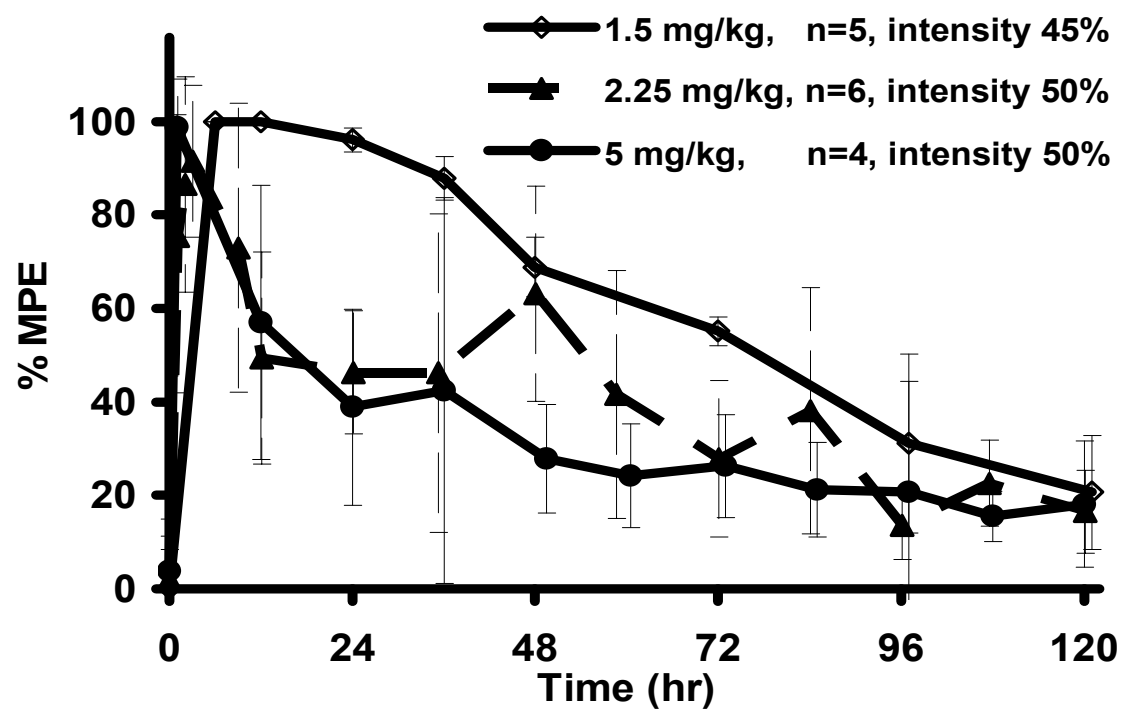

(a)

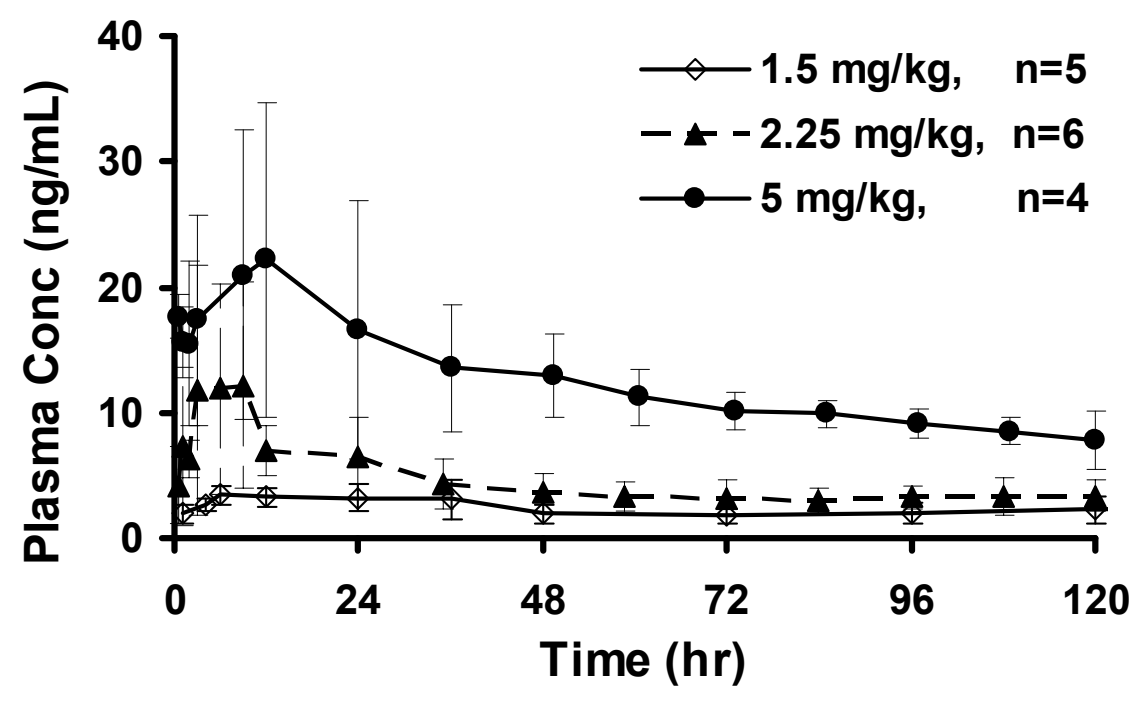

(b)

Figure 2-4. Effect of varying buprenorphine dose on (a) analgesia and (b) buprenorphine plasma concentration in rats after a single subcutaneous injection of the formulations prepared with TBC.

Each data point represents an average of $n$ measurements.

Standard deviation of the measurements is presented as error bars. 


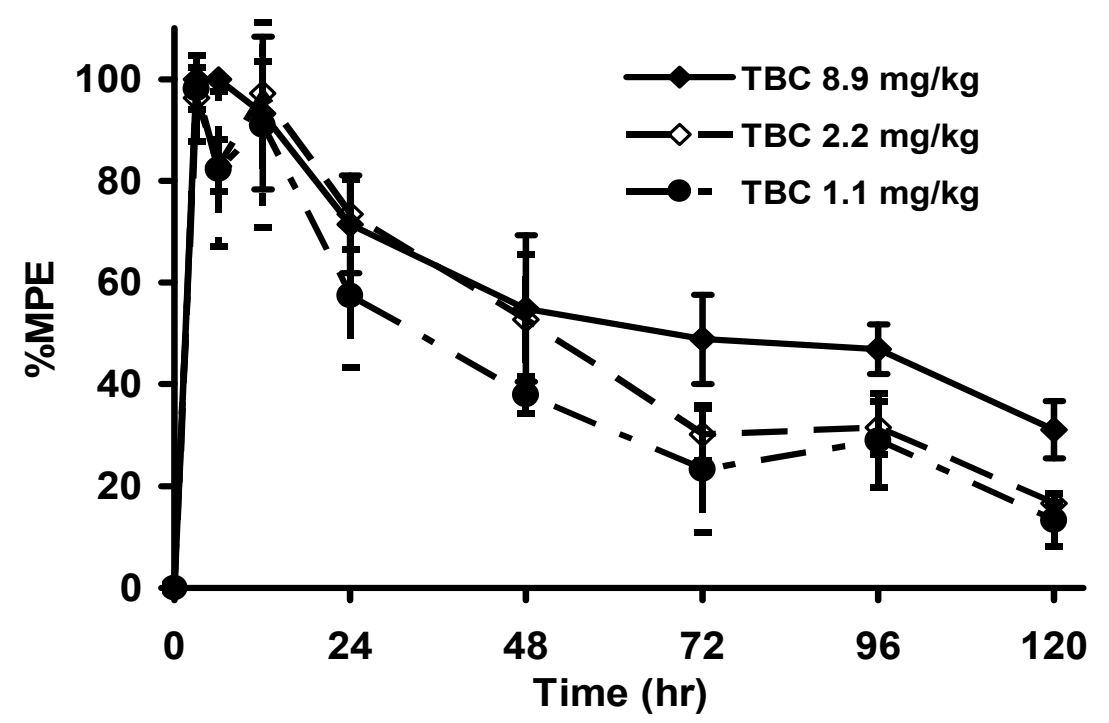

(a)

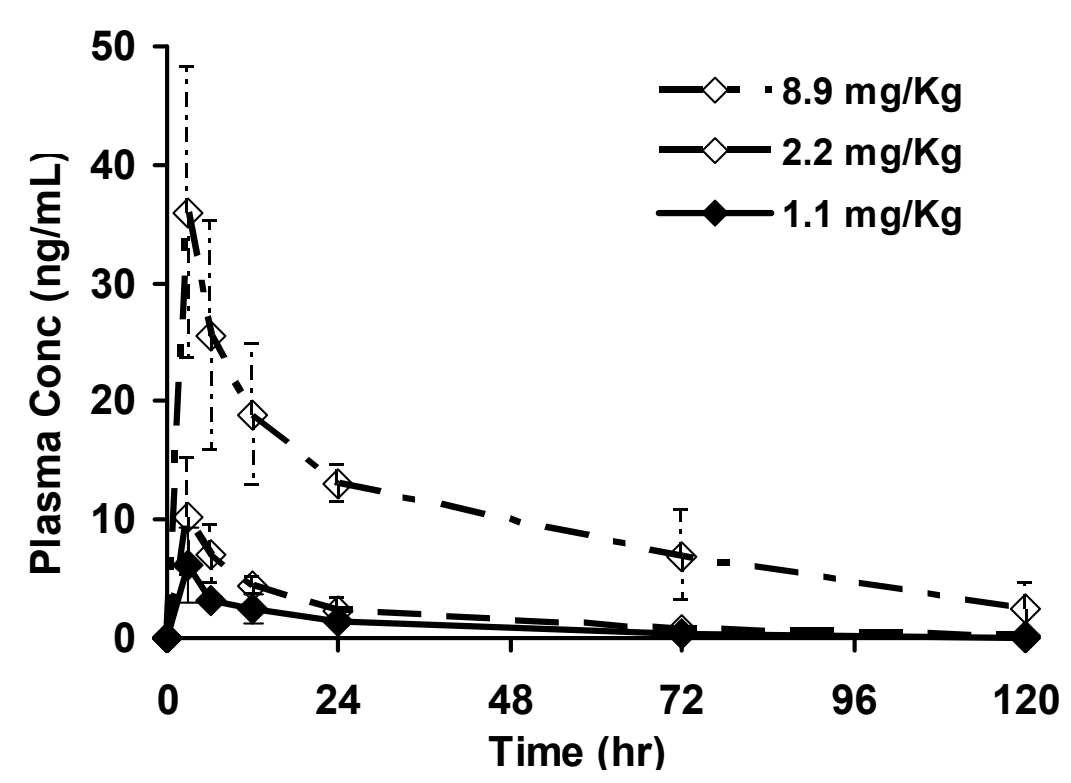

(b)

Figure 2-5. Effect of doses on (a) analgesia $(n=5)$ and (b) buprenorphine plasma concentrations in mice after subcutaneous injection of formulations.

Each data point represents an average of the measurements.

Standard deviation of the measurements is presented as error bars.

Intensity of the tail flick tester during the experiment is $30 \%$. 
hours after a single subcutaneous injection of $1.1 \mathrm{mg} / \mathrm{kg}$ dose. Then the analgesia declined to 57.5\% MPE at 24 hours (1 day) and 13\% MPE at 120 hours (5 days).

Figure 2-5b shows that buprenorphine plasma concentrations increased proportionately as the dose of the drug increased from 1.1 to 2.2 to $8.9 \mathrm{mg} / \mathrm{kg}$. For the 1.1 $\mathrm{mg} / \mathrm{kg}$ dose, the average Cmax was $6.2 \mathrm{ng} / \mathrm{mL}$ at 3 hours, and the average plasma concentration was maintained above $1.4 \mathrm{ng} / \mathrm{mL}$ for 24 hours and above $0.3 \mathrm{ng} / \mathrm{mL}$ for 3 days. For the $2.2 \mathrm{mg} / \mathrm{kg}$ dose, the average Cmax was $10.2 \mathrm{ng} / \mathrm{mL}$ at 3 hours, and the average plasma concentrations was maintained above $0.8 \mathrm{ng} / \mathrm{mL}$ for 3 days and above 0.1 $\mathrm{ng} / \mathrm{mL}$ for 5 days. For the $8.9 \mathrm{mg} / \mathrm{kg}$ dose, the average Cmax was $35.9 \mathrm{ng} / \mathrm{mL}$ at 3 hours, and the average plasma concentration was maintained above $2.5 \mathrm{ng} / \mathrm{mL}$ for 5 days in the animals. No significant difference in the maintenance of analgesia was found at the doses of 8.9 and $2.2 \mathrm{mg} / \mathrm{kg}$ for up to 48 hours after injection, although the drug plasma concentration and AUC of $8.9 \mathrm{mg} / \mathrm{kg}$ dose is significant higher than those of $2.2 \mathrm{mg} / \mathrm{kg}$ dose. This shows that the relationship between drug plasma concentrations and analgesia response are not linear. In this study, 30.1\% MPE can be maintained for 3 days with the drug plasma concentration of $0.8 \mathrm{ng} / \mathrm{mL}$. The bell-shaped dose response curve may explain it and has been demonstrated in mice after subcutaneous injection of buprenorphine hydrochloride. ${ }^{98}$

\subsubsection{Typical photographs of the subcutaneous tissue at the injection site after administration of different formulations in rats and mice}

The toxicity and skin irritation studies of citric acid esters including ATEC and ATBC showed tested citrated were safe to use. ${ }^{95}$ Although there was absence of irritation data on $\mathrm{TBC}$, it is reasonable to expect the low or no irritation properties of TBC because of the structure similarity with ATBC. The injection site views after subcutaneous of the buprenorphine formulations R1 to R3 and M1 to M3 in rats and mice were similar.

Figure 2-6a shows the subcutaneous injection site of the rat after administration of buprenorphine formulation R1 at day 5 . The figure indicated that no formulation remained at the injection site at day 5 after a single subcutaneous administration of buprenorphine formulations prepared with TBC. In addition, no obvious inflammation was observed in most of tested rats at the injection sites. The sign of mild inflammation was observed in 2 out of 15 tested rats. Figure 2-6b also indicated that no formulation remained at the injection site on day 5 after the administration of formulation M2 in mice. Moreover, no obvious inflammation was observed in mice at the injection sites.

\subsubsection{Evaluation of toxicity of citric acid esters in rats}

Figure 2-7 shows the body weight changes of the rats with time after a single subcutaneous injection of $200 \mu \mathrm{L}$ of sterilized citric acid esters (TEC, ATEC, TBC and ATBC) or normal saline solution. No systemic toxicity or significant body weight change $(\mathrm{P}=0.946$, one-way ANOVA) was observed in the rats injected with the citric acid esters compared to those injected with normal saline. Also no abnormal eating and drinking 


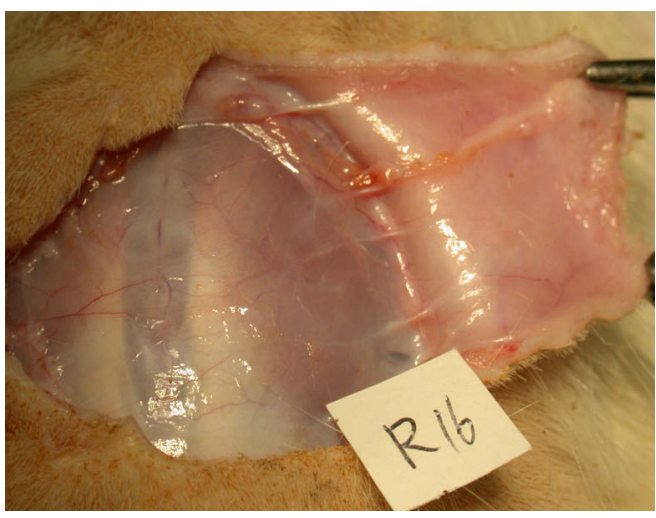

(a) $1.5 \mathrm{mg} / \mathrm{kg}$ (Rat) at day 5

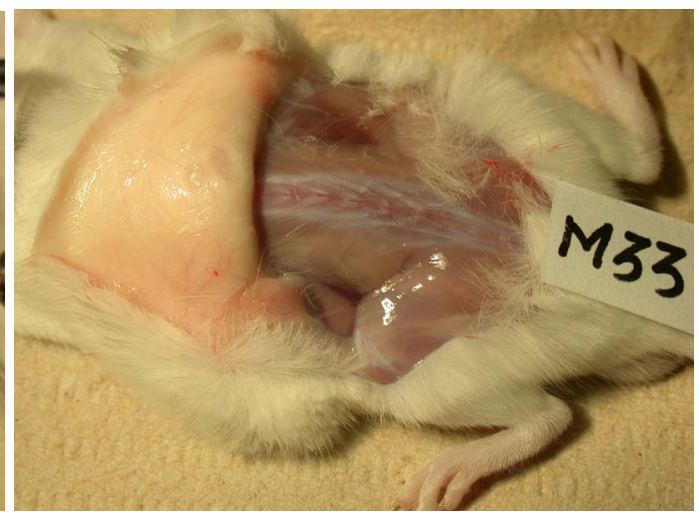

(b) $2.2 \mathrm{mg} / \mathrm{kg}$ (Mouse) at day 5

Figure 2-6. Typical injection site after subcutaneous administration of buprenorphine formulation prepared with TBC in (a) rats and (b) mice at day 5.

Doses in the rat and mouse studies are $1.5 \mathrm{mg} / \mathrm{Kg}$ and $2.2 \mathrm{mg} / \mathrm{Kg}$, respectively. 


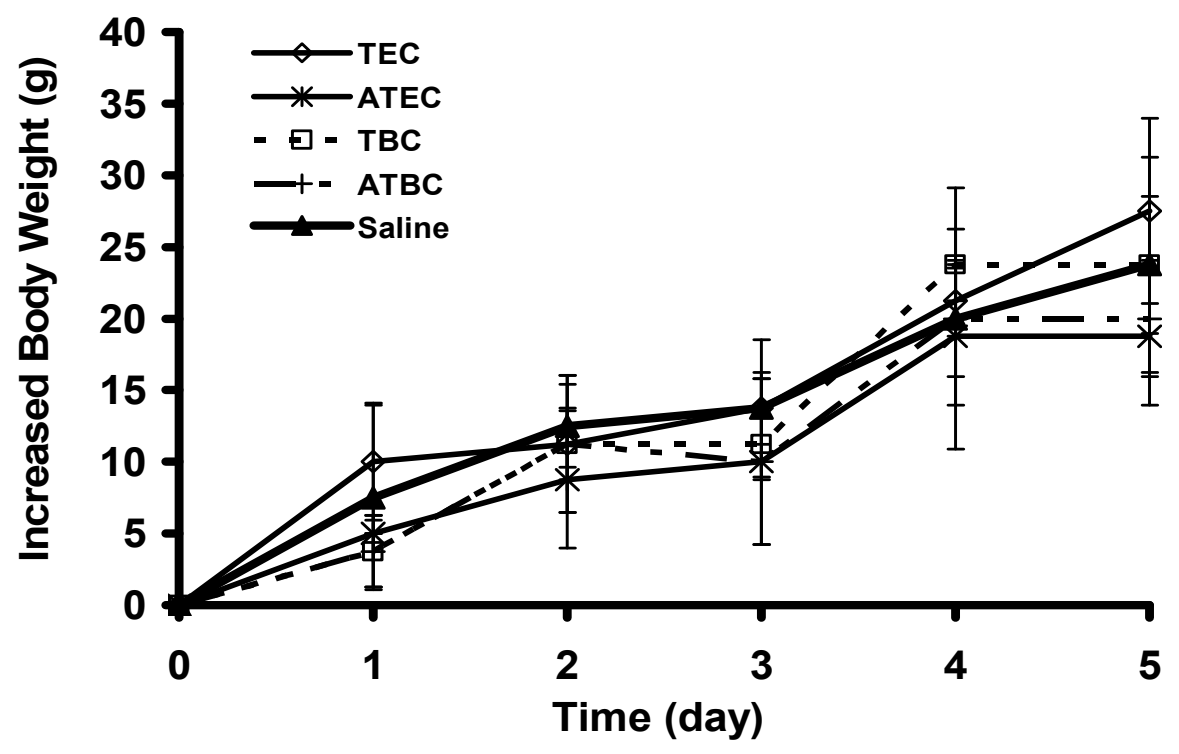

Figure 2-7. Body weight changes of rats after subcutaneous administration of different citric acid esters or normal saline solution.

Each data point represents an average of four measurements.

Standard deviation of four measurements is presented as error bars.

The $\mathrm{P}$ value is 0.946 . 
habits were observed. This is in accord with the results of acute and chronic toxicity studies of TEC, ATEC, TBC and ATBC which indicated that all four tested citric acid esters would not cause damage to the liver, kidney, lungs and spinal cord for the tested animals. ${ }^{99,100}$

\subsection{Conclusions}

Oral administration route is the most convenience delivery method for human, but it, such as putting analgesic in the water for the rodents, runs the risk of inaccurate dosing and that of degradation due to hydrolysis. ${ }^{101}$ Therefore methods for analgesic delivery to rodents are primarily limited to parenteral rather than oral delivery.

Animal welfare regulations require that analgesia should be provided whenever a procedure is performed or a condition is present that is likely to cause pain. Laboratory rats and mice are widely used in research and suffer the pain after the surgery. Opioid analgesics are the primary therapeutic agents used for moderate to severe pain. ${ }^{87}$ Buprenorphine, butorphanol and morphine are three commonly used opioids. Buprenorphine had an intermediate analgesic effect and the longest duration (6 to $8 \mathrm{hrs}$ in rats and 3 to $5 \mathrm{hrs}$ in mice) compared to morphine ( 2 to $3 \mathrm{hrs}$ ) and butorphanol (1 to 2 hrs) in rats and mice. ${ }^{93}$ The Formulary for Laboratory Animals lists the recommended buprenorphine dose as $0.01-0.05 \mathrm{mg} / \mathrm{kg}$ (SC/IV, 2-3 times daily) for rats and 0.05-0.1 $\mathrm{mg} / \mathrm{kg}$ (SC, 2-4 times daily) for mice.

Sustained release analgesic provides the advantages of accurate dosing, reducing the stress of frequent handling and injection, and improving the well-being of research animals. This hydrophobic delivery system has the advantages of easy preparation, simple sterilization by filtration, easy administration (including filling and injection), and cost efficiency. The use of a sustained-release analgesic hydrophobic formulation could decrease the frequency of handling and increase the welfare of animals at low cost.

The solubility, stability and in vitro dissolution studies were performed in vitro to screen the hydrophobic vehicles in this study. Buprenorphine in TBC vehicle showed high solubility, good stability, and desired sustained drug release in vitro performance. In addition, TBC has been used widely in pharmaceutical products and the toxicity data supported that TBC was safe to use at tested amount. Therefore it was selected as the hydrophobic vehicle and tested in rats and mice at different doses. The dosing in PK studies was based on the calculation and prediction from a desired release profile. The PK and PD results showed that a simple long-acting controlled release delivery system of buprenorphine capable of maintaining analgesia in rats and mice for 3 to 5 days after a single subcutaneous administration was developed in this study. 


\section{CHAPTER 3. A NOVEL IN VITRO DISSOLUTION MODEL FOR OILY FORMULATIONS: MODEL DESIGN, RELEASE MECHANISM, AND THE DEVELOPMENT OF LEVEL A IVIVC}

\subsection{Introduction}

Current United States Pharmacopeia (USP) apparatus for in vitro release testing was designed mainly for oral and transdermal products. In contrast, there are no standard regulatory methods for parenteral sustained release products at present. ${ }^{69,70}$ Over the past decade, several methods have been attempted to describe drug release from parenteral products and the current in vitro release methods for parenterals might be divided into four broad categories: sample-and-separate, ${ }^{69,70}$ ultrafiltration, ${ }^{70}$ continuous flow methods, ${ }^{69,70}$ and dialysis techniques including rotating dialysis, ${ }^{71}$ dialysis sac, ${ }^{70}$ and microdialysis. ${ }^{70}$ The advantages and disadvantages of each method were discussed by Diane J. Burgess ${ }^{70}$ and Claus Larsen. ${ }^{69}$ In general, dialysis techniques are considered feasible for the study of drug release from the solutions and suspensions. ${ }^{102,103}$ Amongst these methods, the rotating dialysis cell model has been used to study drug release from oily depot solutions. ${ }^{71}$ It offers the advantages of reproducible results and fast distribution processes. But it has been shown to establish an in vitro-in vivo correlation for just one particular formulation. ${ }^{104}$ In addition, commercially available Float A Lyzer ${ }^{\circledR}$ dialysis tubes can be used as an alternative dialysis model. In contrast to the rotating dialysis cell, it works at much less intensive stirring conditions, resulting in lower drug transport rates. $^{105}$

A successful in vitro dissolution model needs to differentiate the variants within the appropriate formulations with similar drug release mechanism, and any changes with respect to process or manufacturing site. Level A IVIVC can be used to set the critical dissolution specifications and as a surrogate for in vivo bioequivalence. ${ }^{103}$ As regards to quality control as well as formulation development purposes, ${ }^{69,106}$ it is still highly desirable to a develop suitable in vitro release model for parenteral products with the characters of Level A IVIVC and elaboration on drug release mechanism. The development of IVIVC for parenteral products is more difficult than oral dosage forms. Only a few publications had success on IVIVC for parenteral products ${ }^{107}$ and very few examples of IVIVC being developed from more than one formulation. ${ }^{107}$ In addition, for most examples in which IVIVC was stated for parenterals, the mathematical models that describe the drug release have not been provided. ${ }^{107}$ So far there is no successful in vitro dissolution method with the character of Level A IVIVC exists at present for parenteral oily formations. In general, it is accepted that the process of development and validation of IVVIC for parenterals could follow the same as modified release (MR) oral products $^{107}$. The principles of FDA IVIVC MR guidance ${ }^{108}$ for oral products were used to develop level A IVIVC for parenterals in this study.

Long-acting buprenorphine controlled release formulations were developed and tested in this study in order to verify the new dissolution model. The purposes of this 
study are to design, develop and validate the novel dissolution model with the character of level A IVIVC and to elucidate the drug release mechanism of this model.

\subsection{Materials and Methods}

\subsubsection{Materials}

Buprenorphine was obtained from Diosynth Inc. (Morrisville, NC). Tributyl citrate (TBC) and acetyltributyl citrate (ATBC) were obtained from Morflex, Inc. (Greensboro, NC). Heparinized Microvette tubings (Microvette CB 300 LH) were purchased from Sarstedt (Newton, NC). Sodium dodecyl sulfate (SDS) and acetonitrile $(\mathrm{ACN})$ were purchased from Sigma-Aldrich (St. Louis, MO).

\subsubsection{Animals}

Approximately 300 g Sprague Dawley male rats (Charles River Labs, Wilmington, MA) were used in this study. The animals were randomly assigned and group-housed in polycarbonate caging with ad libitum access to food and water. All animal experiments were conducted according to protocols approved by the Institutional Animal Care and Use Committee (IACUC).

\subsubsection{Softwares}

Excel 2003 (Microsoft Office, Redmond, WA), WinNonlin (Pharsight Corporation, Cary, NC), SPSS 17.0 (SPSS Inc., Chicago, IL), and Stella 9.0 (isee systems, Inc., Lebanon, $\mathrm{NH}$ ) were used to process the data generated in this study.

\subsubsection{Analytical methodology for in vitro and in vivo studies}

\subsubsection{Analytical methodology for in vitro studies - HPLC/fluorescence analysis}

HPLC method validation developed to quantitate buprenorphine in samples obtained from in vitro studies has been discussed in Chapter 2. Briefly, discovery C-18 column (2.1 x $100 \mathrm{~mm}$ ) (Supelco, Bellefonte, PA) was used. The mobile phase was composed of a mixture of $51 \mathrm{mM} \mathrm{KH}_{2} \mathrm{PO}_{4}$ buffer and acetonitrile $(\mathrm{ACN})(45: 55, \mathrm{v} / \mathrm{v})$ with the final $\mathrm{pH} 6.65$ adjusted by $10 \mathrm{~N} \mathrm{NaOH}$ solution. The oven temperature was $40^{\circ} \mathrm{C}$ and the flow rate was $0.3 \mathrm{~mL} / \mathrm{min}$. The total run time was 6 minutes. Buprenorphine was detected with fluorescence detector at $\lambda_{\text {Exitation }}=292 \mathrm{~nm}$ and $\lambda_{\text {Emission }}=350 \mathrm{~nm}$. 


\subsubsection{Analytical methodology for in vivo studies - LC-MS/MS method}

Validation of LC/MS/MS method has been discussed in Chapter 2 to quantify the concentrations of buprenorphine in plasma samples obtained from in vivo studies. Briefly, LC/MS/MS system consisted of Shimadzu HPLC systems, a HILIC silica column $(5 \mu \mathrm{m}, 150 \times 4.6 \mathrm{~mm})$ with a guard column $(10 \times 2.1 \mathrm{~mm})$ (Waters Inc., Milford, MA), and API 4000 tandem triple quadrupole mass spectrometer (Applied Biosystems, Foster City, CA). Solution A (ACN : water : formic acid (4.95:95:0.05, v/v/v) with $2 \mathrm{mM}$ ammonium formate) and solution B (ACN : water : formic acid $(95: 4.95: 0.05, \mathrm{v} / \mathrm{v} / \mathrm{v})$ with $2 \mathrm{mM}$ ammonium formate) were used. The time program of the gradient method started with $90 \%$ of Solution B followed by decreasing linearly from $90 \%$ to $40 \%$ within 3.5 minutes. Then it immediately increased to $90 \%$ and maintained at $90 \%$ for 2 minutes. The concentration of Solution B was further decreased linearly from $90 \%$ to $40 \%$ within 0.5 minute, linearly increased to $90 \%$ within 0.5 minute and maintained at $90 \%$ for 4 minutes. The column was operated at room temperature and the flow-rate of the mobile phase was $0.5 \mathrm{~mL} / \mathrm{min}$. Ion transition of 468.3 to 468.3 and 472.3 to 472.3 were selected for buprenorphine and buprenorphine-D4 detection, respectively.

\subsubsection{Formulation preparation}

An appropriate amount of buprenorphine base powder was accurately weighed and dispersed into an appropriate amount of solvent(s) (Table 3-1) in $20 \mathrm{~mL}$ glass scintillation vials. The subsequent suspension was sonicated under $25^{\circ} \mathrm{C}$ until all drug powder was dissolved. The final solution was obtained by filtering the above solution through a $0.22 \mu \mathrm{m}$ syringe filter in a sterile hood and then sealed. The drug concentration was verified by HPLC method. Formulation 3-1b and 3-2 were tested in rats for IVIVC development and all prepared formulations were tested in the designed dissolution model for different purposes.

\subsubsection{In vitro tests}

\subsubsection{In vitro dissolution model design}

When the partition coefficient of the API is below approximately $10,000,{ }^{109}$ it is expected that drug molecules transport from the injection site to the systemic circulation by two steps after intramuscular or subcutaneous administration of oily solutions. ${ }^{103}$ Drug molecules (1) diffuse out of the oily vehicle into the aqueous tissue fluid followed by (2) transporting through the tissue fluid to the systemic circulation. Except in-situ forming gels, ${ }^{103}$ partition between the oily vehicle and the aqueous fluids is the rate limited step and absorption rate has little dependence on vehicle viscosity. ${ }^{103,110}$ The cylinder-inbasket (CIB) apparatus (Figure 3-1) was designed and developed based on this drug absorption mechanism, The CIB apparatus consists of a cylinder, a USP standard 1-Liter vessel, a USP standard cylindrical basket (40-mesh basket in this study), a USP standard 
Table 3-1. The formulations tested in the dissolution model development study.

\begin{tabular}{|c|c|c|c|c|c|}
\hline \multirow{2}{*}{ Formulation } & \multirow{2}{*}{ Solvent(s) } & \multirow{2}{*}{$\begin{array}{c}\text { Concentration of } \\
\text { Buprenorphine } \\
\%(w / w)\end{array}$} & \multicolumn{2}{|c|}{ Tested in } & \multirow{2}{*}{$\begin{array}{c}\text { Dose } \\
\text { (mg/kg) }\end{array}$} \\
\hline & & & In Vitro & In Vivo & \\
\hline $3-1 \mathrm{a}$ & TBC & 0.3 & $\mathrm{x}$ & & \\
\hline $3-1 b$ & $\mathrm{TBC}$ & 0.5 & $\mathrm{x}$ & $\mathrm{x}$ & 3 \\
\hline $3-1 c$ & $\mathrm{TBC}$ & 1.5 & $\mathrm{x}$ & & \\
\hline $3-1 d$ & $\mathrm{TBC}$ & 3 & $\mathrm{x}$ & & \\
\hline $3-2$ & ATBC & 0.5 & $\mathrm{x}$ & $\mathrm{x}$ & 3 \\
\hline $3-3 a$ & TEC:ATEC:TBC 1:1:1 (v/v/v) & 0.5 & $\mathrm{x}$ & $\mathrm{x}$ & 3 \\
\hline $3-3 b$ & TEC:ATEC:TBC 1:1:1 (v/v/v) & 1.3 & $\mathrm{x}$ & & \\
\hline
\end{tabular}




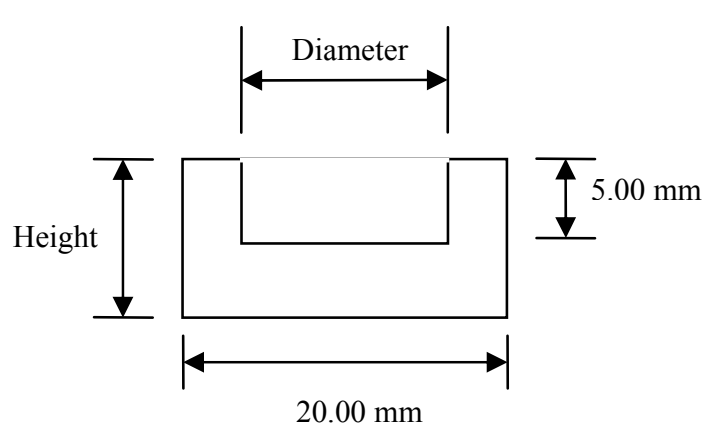

(a)

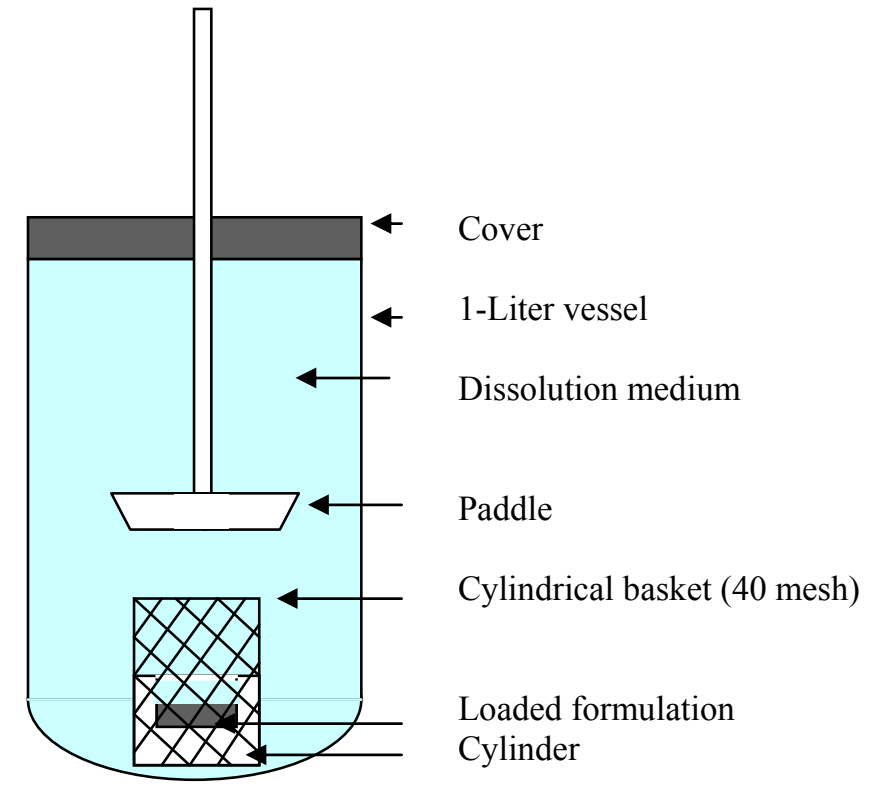

(b)

\begin{tabular}{lcccccc}
\hline \multicolumn{1}{c}{ Cylinder \# } & $\mathbf{\# 1}$ & $\mathbf{\# 2}$ & $\mathbf{\# 3}$ & $\mathbf{\# 4}$ & $\mathbf{\# 5}$ & $\mathbf{\# 6}$ \\
\hline Height $(\mathrm{mm})$ & 12.3 & 12.3 & 12.3 & 24.6 & 24.6 & 24.6 \\
Diameter $(\mathrm{mm})$ & 12.5 & 15.0 & 17.5 & 12.5 & 15.0 & 17.5 \\
\hline
\end{tabular}

Figure 3-1. (a) Cylinder and (b) cylinder-in-basket (CIB) apparatus for in vitro dissolution study. 
paddle, a USP standard dissolution apparatus (Hanson SR8PLUS Dissolution Test Stations, Hanson Research Corp.) and an AutoPlus Autosampler (Hanson Research Corp.). There are two compartments in this CIB model: the small compartment inside the basket and the bulk compartment outside the basket. The solution in the small compartment mimics the body fluid and the media in the bulk compartment mimics the systemic circulation. After loading the formulation into the cavity of the cylinder, the drug molecules were assumed to diffuse into the small compartment followed by transporting to the bulk compartment. The basket was designed to protect the oily vehicle out of the vigorous convection in the bulk compartment and mimic the biological barrier. During the experiment, the oily vehicles were assumed to maintain the constant surface area which benefited the mechanism study. Six different Teflon cylinders (Figure 3-1) were designed, prepared and tested in this study for different purposes. Except the studies to explore the effect of cylinder surface area and height, the cylinder \#5 was used in the rest studies.

\subsubsection{Partition coefficients}

The partition coefficients of buprenorphine between the oily vehicles and dissolution medium were performed in triplicate. According to preliminary studies, solutions of drug in aqueous phase-saturated oily vehicles were allowed to equilibrate with the oil phase-saturated aqueous buffers in the incubator at $100 \mathrm{rpm}$ and $37 \pm 0.5^{\circ} \mathrm{C}$ for at least $48 \mathrm{hr}$. Drug concentrations in the aqueous phase were directly measured by a validated HPLC method. The drug content in the oily phase was determined by diluting 100-fold with ACN : water (70:30, v/v), followed by drug concentrations analysis using HPLC method. The partition coefficients were calculated according to the Equation 3-1.

$$
P=\frac{C_{o i l}}{C_{a q}}
$$

where $C_{o i l}$ and $C_{a q}$ represent the drug concentration in the oily phase and the aqueous phase at the equilibrium state, respectively.

\subsubsection{Short-term stability studies}

Short-term stability of buprenorphine in the dissolution medium at $25^{\circ} \mathrm{C}$ and $40^{\circ} \mathrm{C}$ was evaluated within 5 days. The drug concentration in the tested samples was equal to the value of the maximum amount of drug in tested formulation dissolved in $900 \mathrm{~mL}$ dissolution medium. The sample solution in a $20 \mathrm{~mL}$ scintillation vial wrapped securely with para-film was stored in ovens at $25 \pm 0.5^{\circ} \mathrm{C}$ and $40 \pm 0.5^{\circ} \mathrm{C}$. The study was performed in triplicate at each time point. Drug concentration in the sample at time 0 was used as the reference. The drug content in the samples was tested at predetermined time points using HPLC method. The length of each drug stability study was different and the last time point of the stability study was selected as same as that in dissolution study. 


\subsubsection{In vitro release studies}

The tested formulation was loaded into the cavity of the cylinder followed by covering with the USP basket (40-mesh). The cylinder-in-basket kit was then transferred to the center bottom of a 1-Liter dissolution vessel. The dissolution medium was carefully loaded into the vessel at the rate of $7 \mathrm{~mL} / \mathrm{sec}$ using a modified Hanson Media Mate (Hanson Research Corp.). The paddle blade stirrer was then inserted into the apparatus and located on the top of the basket. The distance between the top of the basket and the bottom of paddle blade was adjusted. Except the study on the effect of paddle distance, the distance was set at $2 \mathrm{~cm}$. The vessels were covered with a special designed lid and tightened with laboratory para-film in order to avoid evaporation of the medium. The paddle and dissolution medium were varied for different purposes and will be specified in the discussion section. Experiments were conducted by triplet. During the dissolution experiment, samples $(1 \mathrm{~mL})$ in bulk dissolution medium were withdrawn from each vessel at predetermined time points. After sampling, $1 \mathrm{~mL}$ blank dissolution medium was refilled into the bulk dissolution medium. Drug content of the samples was measured by a validated HPLC method. The $\mathrm{pH}$ of dissolution medium was adjusted by hydrochloride and sodium hydroxide. The ion strength of dissolution medium calculated by DebyeHückel equation was adjusted by sodium chloride and kept at 0.13 . The percentage of drug released from the solutions was calculated according to the Equation 3-2.

$$
\% \text { Released }=\frac{V_{s} \cdot \sum_{n=1}^{n} C_{n-1}+V_{b} \cdot C_{n}}{M_{\text {total }}} \cdot 100 \%
$$

where $\mathrm{V}_{\mathrm{s}}$ is the sampling volume ( $1 \mathrm{~mL}$ in this study) at different time points and $\mathrm{V}_{\mathrm{b}}$ is the volume of total dissolution medium; $C_{n}$ and $C_{n-1}$ represent the drug concentration in sample $n$ and $n-1 ; M_{\text {total }}$ is the total amount of drug in the tested formulation.

\subsubsection{Discriminating power of the dissolution test}

The drug release profile can be predicted from proposed equation. The discriminatory power of the dissolution test was used to evaluate the difference between predicted and experimental dissolution profiles. The predicted and experimental dissolution profiles were compared using two methods: the difference factor $\left(f_{1}\right)$ and the similarity factor $\left(f_{2}\right)$. The $f_{1}$ factor ${ }^{11}$ measures the percent difference between two curves over all time points and can be calculated by Equation 3-3. The value of $f_{1}$ factor is zero when the test and drug reference profiles are identical and proportionally increases as the dissimilarity of the two dissolution profiles increases.

$$
f_{1}=\left\{\frac{\left[\sum_{t=1}^{n}\left|R_{t}-T_{t}\right|\right]}{\left[\sum_{t=1}^{n} R_{t}\right]}\right\} \times 100
$$


where $n$ is the number of time points, $R_{t}$ and $T_{t}$ are the percent dissolved of the reference and test product, respectively, at each time point. The predicted dissolution profile was used as the reference.

The $\mathrm{f}_{2}$ factor $^{111}$ is a logarithmic reciprocal square root transformation of the sum of squared error and measures the differences between the test and the reference products over all time points and can be calculated by Equation 3-4. This factor is 100 when the test and reference profiles are identical and decreases as the similarity of the two dissolution profiles decreases.

$$
f_{2}=50 \times \log \left\{\left[1+\left(\frac{1}{n}\right) \sum_{t=1}^{n}\left(R_{t}-T_{t}\right)^{2}\right]^{-0.5} \times 100\right\}
$$

\subsubsection{IVIVC development}

\subsubsection{In vitro studies for IVIVC development}

The in vitro drug release of formulation $3-1 \mathrm{~b}$ and 3-2 were conducted in CIB dissolution model. The loading volume $(\sim 0.2 \mathrm{~mL})$ of the oily formulation was the same with the injection volume in rat studies. The mixture of $15 \%(\mathrm{v} / \mathrm{v}) \mathrm{n}$-propyl alcohol (nPA) in Phosphate buffered saline (PBS) solution with $0.15 \%(\mathrm{w} / \mathrm{v})$ sodium dodecyl sulfate (SDS) at pH6.0 was selected as the dissolution medium. The paddle speed was set at 50 rpm and cylinder \#5 was utilized. The amount of drug release at different time point, $\mathrm{M}_{\mathrm{t}}$, was evaluated by validated HPLC method. The linear correlation was explored by SPSS software. The percentage drug release was calculated by Excel.

\subsubsection{In vivo evaluation of buprenorphine solutions in rats for IVIVC development}

FDA recommends that two or more formulations with different drug release rate should be used to develop level A IVIVC. ${ }^{108}$ The data generated in rat studies after subcutaneous injection of formulation 3-1b and 3-2 (Table 3-1) were tested in this study. Formulation 3-1b or 3-2 $(\sim 200 \mu \mathrm{L})$ were subcutaneously administered into male Sprague Dawley (SD) rats (four rats/group) using 23-gauge needle and the syringes were weighed before and after administration. The exact weight of the buprenorphine solution administered was calculated based on the weight. Blood samples $(\sim 0.2 \mathrm{~mL})$ were withdrawn by saphenous vein and collected in heparinized Microvette tubings at predetermined time points within two weeks. The collected blood samples were immediately centrifuged at $14 \mathrm{~K}$ rpm for $7 \mathrm{~min}$ and plasma samples were stored at $-20^{\circ} \mathrm{C}$ until further analysis. The plasma samples were processed and analyzed for drug content using the validated LC/MS/MS method. The average plasma concentration at each time point was reported. 


\subsubsection{Bioavailability of buprenorphine in rats}

An intravenous study was tested in rats to determine the PK parameters of buprenorphine at the dose of $0.05 \mathrm{mg} / \mathrm{kg}$ and described in Chapter 2. The PK parameters $\left(\mathrm{K}_{\mathrm{e}}, \mathrm{AUC}_{0-\mathrm{t}}\right.$, and $\left.\mathrm{AUC}_{0-\infty}\right)$ were calculated by using WinNonlin non-compartment model. The terminal elimination rate constants $\left(K_{\mathrm{e}}\right)$ after i.v. administration were estimated with least-squares regression of values in the terminal log-linear region of plasma concentration-time curves. The areas under the curve (AUC) from time zero to last sampling time $\left(\mathrm{AUC}_{0-\mathrm{t}}\right)$ after drug administration were determined by the logarithmic trapezoidal rule. The area under the curve from time zero to infinity $\left(\mathrm{AUC}_{0-\infty}\right)$ was calculated as $\mathrm{AUC}_{0-\mathrm{t}}+C_{t} / K_{\mathrm{e}}$, where $C_{t}$ is the last detected plasma concentration and $t$ is the time.

Buprenorphine saline solution was subcutaneously injected into four male Sprague Dawley (SD) rats at the dose of $0.05 \mathrm{mg} / \mathrm{kg}$. The syringes were weighed before and after subcutaneous administration and the exact weight of the buprenorphine solution administered was calculated based on the weight. Blood samples $(\sim 0.2 \mathrm{~mL})$ were withdrawn from the saphenous vein with a 25 gauge needle and kept in heparinized Microvette tubings at 0, 5, 15, and $30 \mathrm{~min}$, and 1,2, 4, 6 and $12 \mathrm{hr}$.

The bioavailability (F) was calculated by Equation 3-5 after subcutaneous injection of buprenorphine saline solution at the dose of $0.05 \mathrm{mg} / \mathrm{kg}$.

$$
F=\frac{A U C_{S C} / D_{S C}}{A U C_{i . v .} / D_{i . v .}}
$$

where $\mathrm{AUC}_{\mathrm{SC}}, \mathrm{AUC}_{\mathrm{i} . \mathrm{v} .}$ and $\mathrm{D}_{\mathrm{SC}}, \mathrm{D}_{\mathrm{i} . \mathrm{v} .}$ represent the $\mathrm{AUC}$ and dose after the subcutaneous or intravenous injection.

\subsubsection{Deconvolution method}

The fraction of drug absorbed in vivo (Fa) after subcutaneous injection of formulation 3-1b and 3-2 was determined using the Loo-Reigelman method by Equation 3-6. ${ }^{112}$ The model-dependent pharmacokinetic parameters of $K_{10}, K_{12}$ and $K_{21}$ used in the calculation were calculated using WinNonlin two-compartment model. The deconvolution was accomplished on a spread-sheet in Excel.

$$
\% F a=\frac{\left(X_{A}\right)_{t}}{\left(X_{A}\right)_{\infty}} \times 100=\frac{C_{t}+k_{10} A U C_{0}^{t}+\frac{\left(X_{P}\right)_{t}}{V_{C}}}{k_{10} A U C_{0}^{\infty}} \times 100
$$

where $\left(\mathrm{X}_{\mathrm{A}}\right)_{\mathrm{t}},\left(\mathrm{X}_{\mathrm{A}}\right)_{\infty}$ and $\left(\mathrm{X}_{\mathrm{p}}\right)_{\mathrm{t}}$ represent the amount of drug absorbed at time t, the amount of drug ultimately absorbed, and the amount of drug in the peripheral compartment at time t. $\mathrm{C}_{\mathrm{t}}, \mathrm{K}_{10}$ and $\mathrm{V}_{\mathrm{c}}$ represent the plasma concentration at time $\mathrm{t}$, the apparent first-order 
elimination rate constant of the drug from the central compartment, and the apparent volume of the central compartment. $\mathrm{AUC}_{0-\mathrm{t}}$ and $\mathrm{AUC}_{0-\infty}$ represent area under the plasma concentration-time curve from time zero to the last measurable concentration and from time zero to infinity.

After the calculation of $\mathrm{Fa}$, the drug release amount at different time point in vivo was calculated based on bioavailability and total amount injected. The linear correlation between $\operatorname{Ln}\left[\mathrm{M}_{\text {total }} /\left(\mathrm{M}_{\text {total }}-\mathrm{M}_{\mathrm{t}}\right)\right]$ and time was explored by SPSS software, where $\mathrm{M}_{\text {total }}$ is the total amount of the drug absorbed.

\subsubsection{IVIVC development and validation}

The data generated in the in-vitro and in-vivo studies and linear relationship between $\mathrm{F}_{\text {diss }}$ and $\mathrm{Fa}$ of tested formulations were used in internal and external validation. The relationship between mean percent of drug dissolved in in-vitro dissolution study $\left(\mathrm{F}_{\text {diss }}\right)$ and the mean fraction of drug absorbed in-vivo $(\mathrm{Fa})$ was examined in this study. The $\mathrm{F}_{\text {diss }}$ was determined using the CIB dissolution testing method and $\mathrm{Fa}$ was determined by Loo-Riegelman method. The linear regression analysis was applied to explore the relationship between $\mathrm{F}_{\text {diss }}$ and $\mathrm{Fa}$. The values of coefficient of determination $\left(\mathrm{R}^{2}\right)$, slope and intercept were calculated, respectively.

The internal predictability of the IVIVC was evaluated by comparing predicted plasma profiles with the actual plasma profiles of formulation 3-1b and 3-2. The prediction of the plasma drug concentration and AUC was accomplished using IVIVC toolkit of Stella 9.0 (isee systems, Inc.) software. ${ }^{113}$ The $\mathrm{pK}$ parameters of buprenorphine in rats, the linear relationship generated in IVIVC development, and the mean in-vitro dissolution data $\left(\mathrm{F}_{\text {diss }}\right)$ of each examined formulation were input into the software. The observed and IVIVC model-predicted Cmax and AUC values for each formulation were used to access the predictability of the correlation. The percent prediction errors $(\% \mathrm{PE})$ for $\mathrm{C}_{\max }$ and $\mathrm{AUC}$ were used to evaluate prediction and calculated by Equations 3-7 and 3-8. ${ }^{108}$

$$
\begin{aligned}
& \% P E_{\max }=\frac{C_{\max }(o b s)-C_{\max }(\text { pred })}{C_{\max }(o b s)} \times 100 \\
& \% P E=\frac{A U C(o b s)-A U C(\text { pred })}{A U C(o b s)} \times 100
\end{aligned}
$$

where $\mathrm{C}_{\max }$ (obs), $\mathrm{C}_{\max }$ (pred) and $\mathrm{AUC}(\mathrm{obs}), \mathrm{AUC}(\mathrm{pred})$ are the observed and IVIVC model predicted maximum plasma concentrations and AUC, respectively.

The external validation was accomplished by comparing predicted plasma profiles with the actual plasma profiles of formulation 3-3a. The pK parameters of buprenorphine in rats, the linear relationship generated in IVIVC development, and the mean in-vitro 
dissolution data $\left(\mathrm{F}_{\text {diss }}\right)$ of the formulation were input into Stella to predict the drug release profile. The same dissolution conditions were applied in in-vitro study. The percent prediction errors $(\% \mathrm{PE})$ for $\mathrm{C}_{\max }$ and $\mathrm{AUC}$ were also used to evaluate prediction.

\subsection{Results and Discussion}

\subsubsection{In vitro studies}

Unlike the solid formulations, the surface of the parenteral formulations is easy to be disrupted by other factors, such as agitation of the dissolution medium. The variance of the surface of the parenteral formulations is one of the major reasons caused the unpredictable drug release. According to the preliminary studies, the high variance could be generated on the steps of the loading of the dissolution medium and by the agitation of the dissolution medium. In this study, the Hanson Media Mate (Hanson Research Corp.) was used to load the dissolution media at the rate of $7 \mathrm{~mL} / \mathrm{sec}$. A special designed tubing was used to conduct the medium solution to the wall of the vessel during the loading process. In addition, the agitation generated by paddle in the bulk solution can cause the high variation of the surface of liquid formulation during the dissolution test. Therefore the effect of paddle position, paddle speed, and cylinder diameter were investigated by formulation 3-1c and dissolution medium of $10 \%(\mathrm{v} / \mathrm{v})$ iso-propyl alcohol in PBS with $0.05 \%$ SDS at $\mathrm{pH} 7.4$ in this study.

\subsubsection{Effect of paddle position}

One of the major problems of in vitro dissolution model development for the parental formulations is the high variation generated during the experiment. In this CIB dissolution model, the basket was designed to protect the liquid formulation out of the effect by the agitation of the dissolution medium. The USP standard 40-mesh basket is selected to protect the liquid formulation. The distance $(0.5$ and $3 \mathrm{~cm})$ between the bottle of paddle blade and the top of the basket was tested on the condition of high agitation (150 rpm) to explore the effect of the paddle position. Two cylinders, \#5 and \#6, with different diameter $(15.0 \mathrm{~mm}$ and $17.5 \mathrm{~mm})$ were tested. If the 40 -mesh basket couldn't protect the liquid formulation, the high variance was expected and the drug release rate tested in two different conditions with different distances should vary because of the different convection environment at the liquid/medium interface.

Figure 3-2 shows the release profiles of buprenorphine from oily vehicle in the CIB dissolution model by changing the paddle location. The $\mathrm{f}_{2}$ values are 81.2/87.4 and $\mathrm{f}_{1}$ values are 12.4/6.1 for the results generated from cylinder \#5 and \#6, respectively. It indicates that there is no significantly difference of the dissolution rate for the test formulation by changing the paddle position at $150 \mathrm{rpm}$. The drug release rate is not affected by the vigorous convection outside the basket under the tested formulation and conditions. In addition, the less variance of dissolution results using CIB model was 

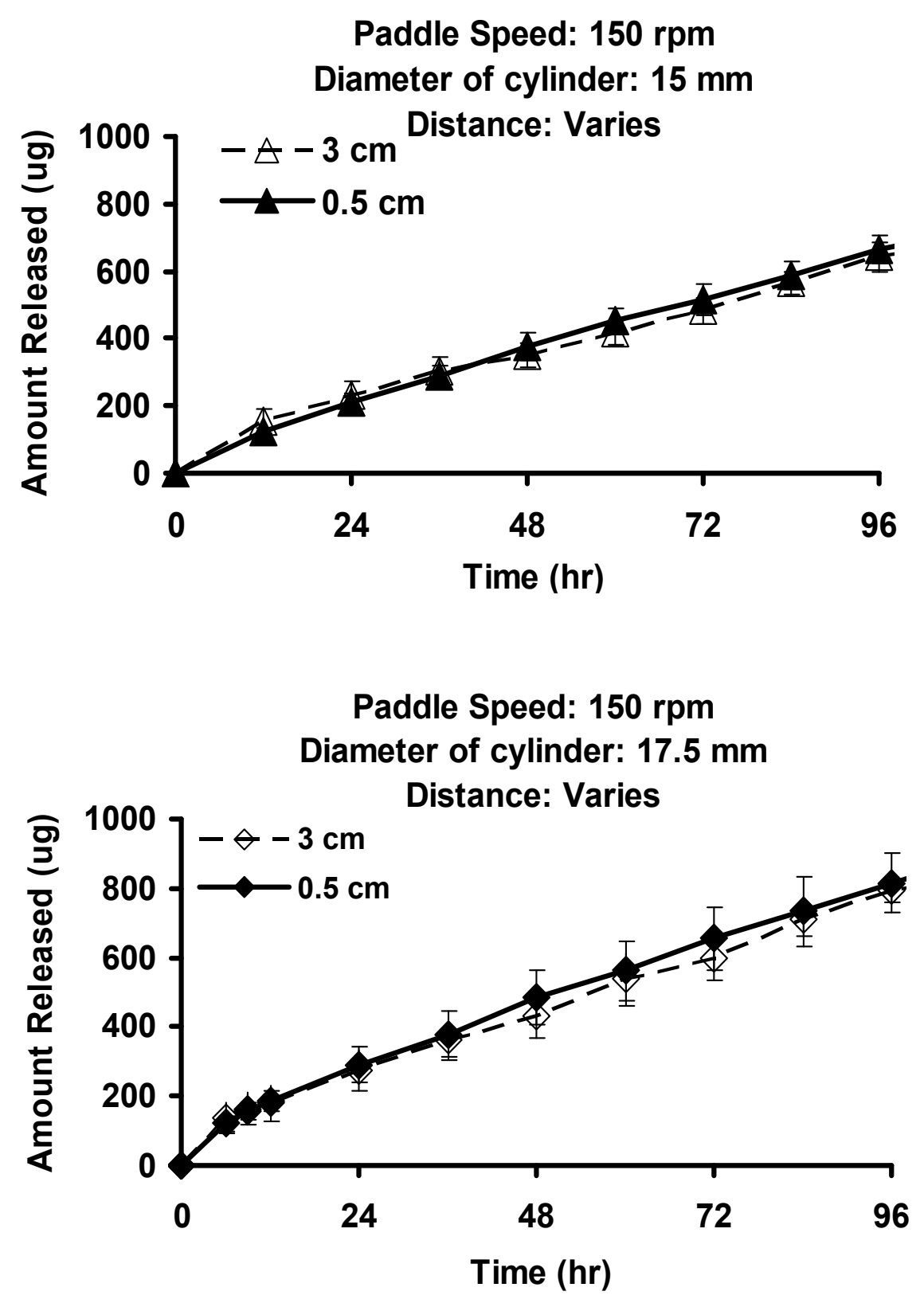

Figure 3-2. The effect of paddle position on the release amount of buprenorphine in CIB dissolution model.

$\mathrm{n}=3$.

Data are presented as mean $\pm \mathrm{SD}(\mu \mathrm{g})$.

Tested formulation was formulation 3-1c. 
observed, which indicated that the 40 -mesh basket can protect the oily phase from the vigorous convection in the bulk solution.

\subsubsection{Effect of paddle speed}

The agitation of the medium could be the other reason which causes the high variation in the CIB model, so the effect of paddle speed was explored at the levels of 100 and $150 \mathrm{rpm}$. Figure 3-3 shows that drug release rate is noticeably faster at $150 \mathrm{rpm}$ than that observed at $100 \mathrm{rpm}$ with low variance. This indicates that drug release rate increases at the higher speed. In the CIB dissolution model, it is expected that drug molecules first diffuse into the small compartment and then diffuse out from small compartment to the bulk compartment. Different paddle speed will affect the convection flow in the bulk media. Vigorous agitation can help drug concentration to achieve the equilibrium between the media inside and outside of the basket. Higher paddle speed is expected to give a more vigorous agitation and to achieve equilibrium in a shorter time, resulting in higher concentration gradient. Therefore, the drug concentration in the bulk media will achieve equilibrium faster and drug release at higher paddle speed gives faster release rate.

\subsubsection{Effect of cylinder height}

Two different sets of cylinders were used. The first set (cylinder \#2 and \#3) has the height of $12.3 \mathrm{~mm}$ with the diameter of $15 \mathrm{~mm}$ and $17.5 \mathrm{~mm}$. The second set (cylinder \#5 and \#6) has the height of $24.6 \mathrm{~mm}$ with the different diameter. Other parameters of the cylinder are the same and the paddle speed during the dissolution study was set at $100 \mathrm{rpm}$. Figure 3-4 shows that the amount of drug release using the $12.3 \mathrm{~mm}$ height cylinder is faster than that using the $24.6 \mathrm{~mm}$ height cylinder. The volume of the cylinder with the height of $12.3 \mathrm{~mm}$ is less than that of the cylinder with the height of $24.6 \mathrm{~mm}$. When $12.3 \mathrm{~mm}$ height cylinder is covered with the basket, the volume of dissolution medium inside the basket (volume of small compartment in CIB model) is relatively larger than that using $24.6 \mathrm{~mm}$ height. When the same amount of drug is diffused out of the oil vehicle into the small compartment, the drug concentration in the small compartment will be relatively lower using $12.3 \mathrm{~mm}$ height cylinder compared to that using $24.6 \mathrm{~mm}$ height cylinder if the drug release rate is the same. And the concentration gradient will be relatively higher by using $12.3 \mathrm{~mm}$ height cylinder. Therefore the height of cylinder could affect the drug release rate and the cylinders with different height should be carefully selected for different purposes.

\subsubsection{Effect of diameter of cylinder}

After loading the oily formulation into the cavity of the cylinder, the surface area between aqueous phase and oily vehicle is assumed to the surface area of the cylinder 

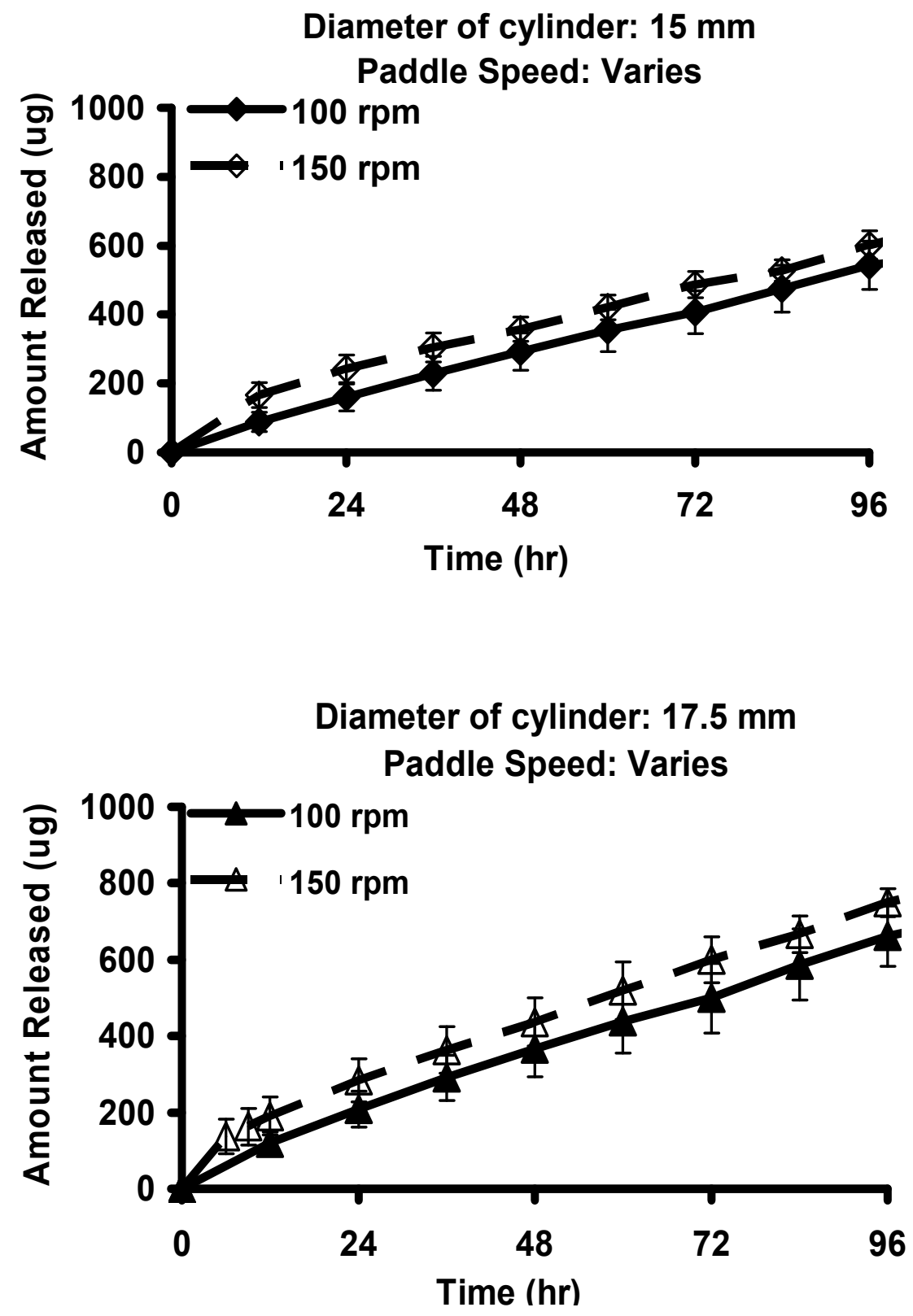

Figure 3-3. The effect of paddle speed on the release amount of buprenorphine in CIB dissolution model.

$\mathrm{n}=3$.

Data are presented as mean $\pm \mathrm{SD}(\mu \mathrm{g})$.

Tested formulation was formulation 3-1c. 

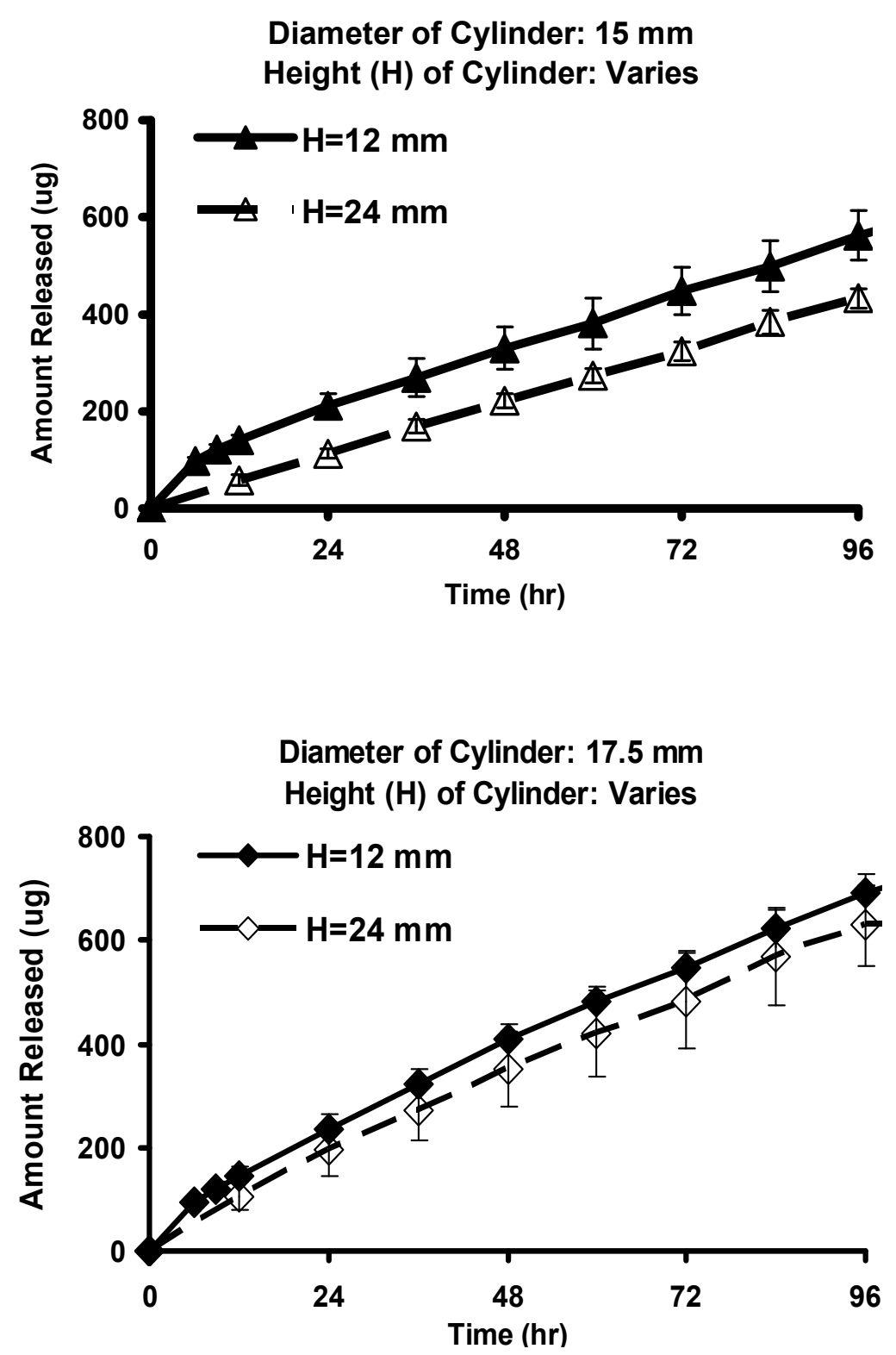

Figure 3-4. The effect of cylinder height on the release amount of buprenorphine in CIB dissolution model.

$\mathrm{n}=3$.

Data are presented as mean $\pm \mathrm{SD}(\mu \mathrm{g})$.

Tested formulation was formulation 3-1c. 
cavity. The cylinder \#1,\#2 and \#3 with different surface area were used in this test and the paddle speed was set at $100 \mathrm{rpm}$. The cylinder diameter aggrandizement from cylinder \#1, \#2 to \#3 also increased the exposed surface area between oil formulation and dissolution medium. Figure 3-5 shows that drug release rate increases with the cylinder surface diameter. By increasing the surface area of cylinder, more drug molecules can diffuse out of oily phase to the aqueous phase at unit time. According to the Equation 3-9, the slope was calculated by plotting $\operatorname{Ln}\left[\mathrm{C}_{0} \mathrm{~V}_{0} /\left(\mathrm{C}_{0} \mathrm{~V}_{0}-\mathrm{M}_{\mathrm{t}}\right)\right]$ versus time. The experimental release rates $\left(\mathrm{K}_{\mathrm{app}}\right)$ for each cylinder are $0.0321,0.0463$ and 0.0625 day $^{-1}$, respectively, with the ratio of $0.69: 1: 1.35$. The ratio of the surface area $\left(\pi r^{2}\right)$ of three cylinders is 0.69:1:1.36. The result is consistent with the Equation 3-9, which indicates the release rate is proportional to the surface area when there is no change in the other parameters. The derivation and details of Equation 3-9 will be discussed in Section 3.3.2.1.

$$
\operatorname{Ln}\left(C_{0} V_{0}\right)-\operatorname{Ln}\left(C_{0} V_{0}-M_{t}\right)=\frac{D S}{h V_{0} P} t=\frac{K S}{V_{0} P} t=K_{a p p} t
$$

where $\mathrm{C}_{0}$ and $\mathrm{V}_{0}$ represent initial drug concentration and initial volume of loaded formulation. $\mathrm{M}_{\mathrm{t}}$ represents the amount of drug at different time in aqueous phase. $\mathrm{D}, \mathrm{h}$ and $\mathrm{S}$ represent diffusion coefficient, the length of diffusion layer, and oil-water interface area. The $\mathrm{K}$ is the ratio of $\mathrm{D}$ divided by $\mathrm{h}$ and $\mathrm{K}_{\mathrm{app}}$ is the apparent rate constant.

\subsubsection{Drug release mechanism}

Drug release mechanism in the CIB dissolution model was explored in this study. The in vitro CIB model was designed to mimic the in vivo drug absorption mechanism after subcutaneous or intramuscular injection. There are two compartments in the CIB model. The small compartment (1) inside the basket and the large compartment (2) outside the basket mimic the body fluid at the injection site and the systemic circulation, respectively. In order to explore drug release mechanism in CIB model, the assumption was made that drug molecules in the oily vehicle diffused into the aqueous dissolution medium and the kinetic equilibrium can be achieved instantly at the interface between the oily vehicle and dissolution medium, although the equilibrium between the oily phase and the bulk solutions may take a few hours to reach in the partition coefficient test. Therefore the drug concentration at the interface between the oily vehicle and dissolution medium can be calculated by Equation 3-10.

$$
P=\frac{C_{o i l}}{C_{a q}}=\frac{C_{t}^{o i l}}{C_{t}^{a q-\text { inter }}}
$$

where $\mathrm{C}_{\mathrm{t}}{ }^{\text {oil }}$ and $\mathrm{C}_{\mathrm{t}}^{\text {aq-inter }}$ are the drug concentration at different time points in the oily phase and the aqueous dissolution medium at the oil-water interface, respectively. $\mathrm{P}$ is the partition coefficient between oily phase and dissolution medium. Both $C_{t}{ }^{\text {il }}$ and $C_{t}{ }^{\text {aq-inter }}$ 


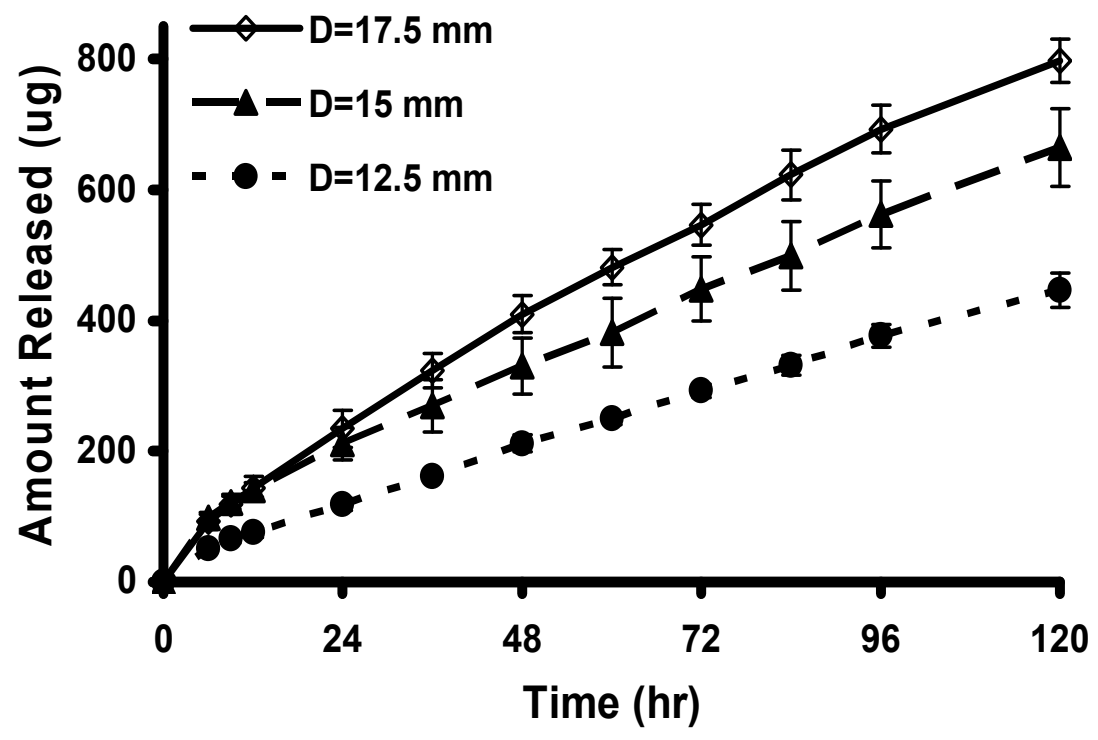

Figure 3-5. The effect of cylinder diameter on the release amount of buprenorphine in CIB dissolution model.

$\mathrm{D}$ is the cylinder diameter.

$\mathrm{n}=3$.

Data are presented as mean $\pm \mathrm{SD}(\mu \mathrm{g})$.

Tested formulation was formulation 3-1c. 
are the variables, but the ratio $\mathrm{P}$ is a constant. The partition coefficient of the drug between oily vehicle and dissolution medium is calculated by measuring the drug concentration in two phases at equilibrium state. Based on the assumption and the concept of Whitney-Noyes equation, the overall drug release in this dissolution model can be calculated from Equation 3-9.

\subsubsection{Derivation of mathematical Equation 3-9}

Noyes-Whitney equation describes the drug release from the solid dosage form, while drug release from the oily vehicle is more complicated. In the solid dosage form, Noyes-Whitney equation shows that concentration gradient is the driving force for drug to diffuse into the aqueous phase. In the sink condition, the drug concentration in the aqueous phase may be ignored, otherwise it can't. Drug release from oily phase to aqueous phase in CIB dissolution model is the diffusion-controlled process and can be illustrated as following.

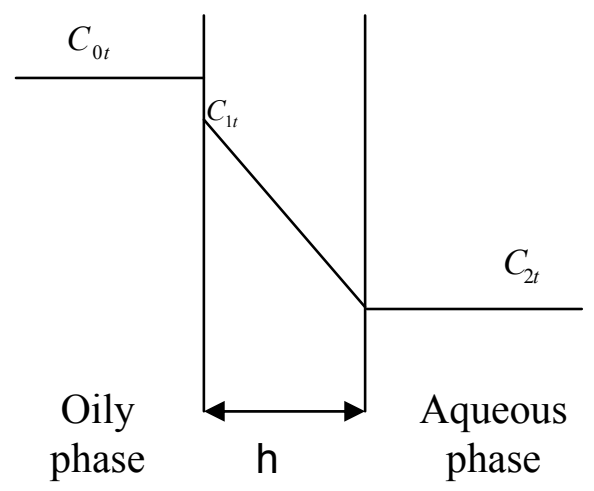

where $\mathrm{C}_{0 \mathrm{t}}, \mathrm{C}_{1 \mathrm{t}}$, and $\mathrm{C}_{2 \mathrm{t}}$ represent drug concentration in oily phase, at the interface, and in the bulk aqueous phase at different time points.

According to aforementioned assumption, drug molecules in the oily vehicle diffuse into the aqueous dissolution medium and the kinetic equilibrium can be achieved instantly at the interface and the drug concentration at the interface between the oily vehicle and dissolution medium can be calculated by Equations 3-1 and 3-10.

During the drug release process, oily vehicle in the formulation could (1) dissolve or (2) partly dissolve in the aqueous phase. Both conditions were considered in the development of the drug mechanism. 


\subsection{Oily phase doesn't dissolve in aqueous phase}

Under the sink condition

$$
\begin{aligned}
& C_{1 t}=\frac{C_{0 t}}{P}=\frac{1}{P}\left(\frac{C_{0} V_{0}-M_{t}}{V_{0}}\right)=\frac{1}{P}\left(C_{0}-\frac{M_{t}}{V_{0}}\right) \\
& d M=\frac{D S}{h}\left(C_{1 t}-C_{2 t}\right) d t=\frac{D S}{h} C_{1 t} d t=\frac{D S}{P h}\left(C_{0}-\frac{M_{t}}{V_{0}}\right) d t
\end{aligned}
$$

where $\mathrm{C}_{0}$ and $\mathrm{V}_{0}$ are constants and represent initial drug concentration and initial volume in the oily phase. $\mathrm{M}_{\mathrm{t}}$ represents the amount of drug at different time pints in aqueous phase.

After solving the differential equation, following Equation 3-9 was derived to describe the drug release from insoluble oily vehicle to the aqueous solution in the sink condition.

$$
\frac{D S}{h V_{0} P} t=\operatorname{Ln}\left(C_{0} V_{0}\right)-\operatorname{Ln}\left(C_{0} V_{0}-M_{t}\right)
$$

Under the non-sink condition

$$
\begin{aligned}
& d M=\frac{D S}{h}\left(C_{1 t}-C_{2 t}\right) d t \\
& C_{1 t}=P^{-1} C_{0 t}=P^{-1}\left(C_{0}-\frac{M_{t}}{V_{0}}\right) \\
& C_{2 t}=\frac{M_{t}}{V_{a}}
\end{aligned}
$$

where $\mathrm{V}_{\mathrm{a}}$ is the volume of the aqueous phase.

After solving the differential equation, Equation 3-11 is obtained.

$$
\frac{D S}{h V_{0} V_{a}} t=\frac{\operatorname{Ln}\left(P^{-1} C_{0} V_{0} V_{a}\right)-\operatorname{Ln}\left[P^{-1} C_{0} V_{0} V_{a}-\left(P^{-1} V_{a}+V_{0}\right) M_{t}\right]}{P^{-1} V_{a}+V_{0}}
$$

The $\mathrm{V}_{0}$ is usually $0.2 \mathrm{~mL}$ and $\mathrm{Va}$ is $900 \mathrm{~mL}$ in this study. It indicates when $\mathrm{P}^{-1} \mathrm{~V}_{\mathrm{a}}$ $>>V_{0}$ or $P$ is less than 4500, Equation 3-11 can be simplified to Equation 3-9.

$$
\frac{D S}{h V_{0} P} t=\operatorname{Ln}\left(C_{0} V_{0}\right)-\operatorname{Ln}\left(C_{0} V_{0}-M_{t}\right) \quad \text { when } \mathrm{P}^{-1} \mathrm{~V}_{\mathrm{a}}>>\mathrm{V}_{0}
$$




\subsection{Oily phase partially dissolves in aqueous phase}

If oily phase partially dissolves in aqueous phase, $\mathrm{V}_{0}$ will not be the constant and the release of the oily vehicle will affect the release of drug. The release of oil and drug molecules is considered as independent. The release of oil will decrease the volume of oily vehicle and increase the drug concentration in the oily phase. Therefore the drug release rate will increase. According to the Noyes-Whitney equation, the release of oily vehicle can be described as:

$$
\begin{aligned}
& \frac{d M^{\prime}}{d t}=\frac{D^{\prime} S^{\prime}}{h^{\prime}}\left(C_{s}{ }^{\prime}-C_{2}{ }^{\prime}\right)=\frac{D^{\prime} S^{\prime}}{h^{\prime}}\left(C_{s}{ }^{\prime}-\frac{M_{t}{ }^{\prime}}{V_{a}}\right)=\frac{D^{\prime} S^{\prime}}{V_{a} h^{\prime}}\left(V_{a} C_{s}{ }^{\prime}-M_{t}{ }^{\prime}\right) \\
& \Rightarrow \operatorname{Ln}\left(V_{a} C_{s}{ }^{\prime}-M_{t}{ }^{\prime}\right)-\operatorname{Ln}\left(V_{a} C_{s}{ }^{\prime}\right)=-\frac{D^{\prime} S^{\prime}}{V_{a} h^{\prime}} t \\
& \Rightarrow M_{t}{ }^{\prime}=V_{a} C_{s}{ }^{\prime}\left(1-e^{-\frac{D^{\prime} S^{\prime}}{V_{a} h^{\prime}}}\right)
\end{aligned}
$$

where $\mathrm{C}_{\mathrm{s}}{ }^{\prime}, \mathrm{C}_{2}$ ' represent saturated concentration of oil and concentration of oil in the aqueous phase, respectively. $\mathrm{M}_{\mathrm{t}}$ ' represents the amount of oily vehicle at different time in aqueous phase. D', h' and S' represent diffusion coefficient, the length of diffusion layer, and oil-water interface area of the oil vehicle.

Then the release of drug molecules can be described as:

$$
\begin{aligned}
& d M=\frac{D S}{h}\left(C_{1 t}-C_{2 t}\right) d t=\frac{D S}{h}\left[P^{-1}\left(\frac{C_{0} V_{0}-M_{t}}{V_{0 t}}\right)-\frac{M_{t}}{V_{a}}\right] d t=\frac{D S}{h V_{0 t} V_{a}}\left(P^{-1} C_{0} V_{0} V_{a}-P^{-1} M_{t} V_{a}-M_{t} V_{0 t}\right) d t \\
& \Rightarrow \frac{1}{P^{-1} C_{0} V_{0} V_{a}-P^{-1} V_{a} M_{t}} d M=\frac{D S}{h V_{0 t} V_{a}} d t \quad \text { when } P^{-1} V_{a} \gg V_{0 t} \\
& \Rightarrow \frac{1}{C_{0} V_{0}-M_{t}} d M=\frac{D S}{h P V_{0 t}} d t
\end{aligned}
$$

where $\mathrm{V}_{0 \mathrm{t}}$ is the volume of oily phase at different points.

$$
\begin{aligned}
& \left.V_{0 t}=\frac{M_{\text {total }}{ }^{\prime}-M_{t}{ }^{\prime}}{d^{\prime}}=\frac{M_{\text {total }}{ }^{\prime}-V_{a} C_{s}{ }^{\prime}\left(1-e^{-\frac{D^{\prime} S^{\prime}}{V_{a} h^{\prime}}}\right)}{d^{\prime}}\right) \\
& \frac{1}{C_{0} V_{0}-M_{t}} d M=\frac{D S d^{\prime}}{h P V_{a} C_{s}{ }^{\prime}} \frac{1}{\frac{M_{\text {total }}{ }^{\prime}}{V_{a} C_{s}{ }^{\prime}}-\left(1-e^{\left.-\frac{D^{\prime} S^{\prime} h^{\prime} h^{\prime}}{V^{\prime}}\right)} d t\right.}=\frac{\frac{D S d^{\prime}}{h P V_{a} C_{s}{ }^{\prime}}}{\left(\frac{M_{\text {total }}{ }^{\prime}}{V_{a} C_{s}{ }^{\prime}}-1\right)+e^{-\frac{D^{\prime} S^{\prime} h^{\prime} t^{t}}{V^{\prime}}}} d t
\end{aligned}
$$

where $\mathrm{d}^{\prime}$ is the density of oil and $\mathrm{M}_{\text {total }}$ ' represents the total amount of oily vehicle.

After dissolving Equation 3-12, Equation 3-13 can be obtained. 


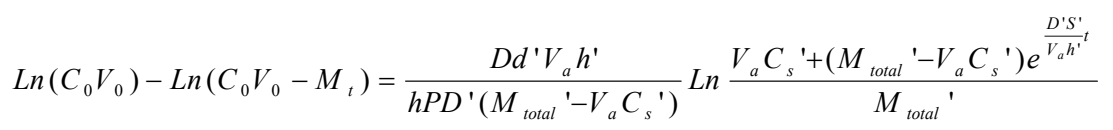

If the oily vehicle is insoluble $\left(\mathrm{Cs}^{\prime} \approx 0\right)$ or partly soluble $\left(\mathrm{M}_{\mathrm{total}}{ }^{\prime}>>\mathrm{V}_{\mathrm{a}} \mathrm{C}_{\mathrm{s}}{ }^{\prime}\right)$ in the aqueous phase, Equation 3-13 can be simplified to the following Equation 3-9.

$$
\operatorname{Ln}\left(C_{0} V_{0}\right)-\operatorname{Ln}\left(C_{0} V_{0}-M_{t}\right)=\frac{D S^{\prime}}{h P V_{0}} t=\frac{D S}{h P V_{0}} t
$$

Oily vehicles usually are insoluble in the aqueous phase and the tested oily vehicle in this study, TBC, is insoluble in water. Although the increasing amount of surfactant or cosolvent and the adjustment of $\mathrm{pH}$ in the dissolution medium could increase the solubility of oily vehicle in the medium. But until the majority of the oily vehicle is insoluble in the aqueous phase, Equation 3-9 can be applied. Therefore, Equation 3-9 is used to describe the drug release from TBC formulations in this study.

\subsubsection{Verification of mathematical equation}

There are seven parameters in Equation 3-9. Amongst them, $M_{t}$ is the variable and can be measured by HPLC. Partition coefficient, P, can be calculated by measuring drug concentration between dissolution medium and oily vehicle at equilibrium state. $\mathrm{K}$ is related to the diffusion coefficient, $\mathrm{D}$, and the length of diffusion layer, h. For the given formulation and dissolution medium, the $\mathrm{P}$ and $\mathrm{K}$ could be considered as the constant. In order to verify this equation, the rest parameters, $\mathrm{C}_{0}, \mathrm{~V}_{0}$, and/or $\mathrm{S}$, were challenged. The verification procedures of Equation 3-9 were performed as follows.

Step 1. Run the dissolution test and calculate $\mathrm{K}$ for the given oily vehicle and dissolution medium.

1. Measure the partition coefficient $P$ between oily vehicle and dissolution medium at equilibrium state

2. Select the cylinder and calculate the $\mathrm{S}$ of the cylinder cavity

3. Prepare the oily formulation and calculate the initial drug concentration $\mathrm{C}_{0}$

4. Weigh the formulation after loading it into the cavity of the cylinder and then calculate the formulation volume $\mathrm{V}_{0}$.

5. Run the dissolution test and measure the released drug amount at predetermined time points, $\mathrm{M}_{\mathrm{t}}$.

6. Calculate the $\mathrm{K}$ from the slope $\mathrm{K}_{\text {app }}$ by plotting $\operatorname{Ln}\left(\mathrm{C}_{0} \mathrm{~V}_{0}\right)-\operatorname{Ln}\left(\mathrm{C}_{0} \mathrm{~V}_{0}-\mathrm{M}_{\mathrm{t}}\right)$ versus time.

Step 2. A new in vitro test was conducted using the same oily vehicle, dissolution medium, and other dissolution conditions as the initial dissolution study in step 1. Since the dissolution conditions didn't change, the initial and new dissolution study should 
share the same $\mathrm{K}$ value which was calculated from initial dissolution study. From the calculated $\mathrm{K}$, the amount drug release could be predicted by challenging variables, $\mathrm{C}_{0}$, $\mathrm{V}_{0}$, and/or $\mathrm{S}$, and calculated by Equation 3-14 derived from Equation 3-9. The predicted results were compared with the experimental data. The variance between experimental and predicted data was compared by calculating $\mathrm{f}_{1}$ and $\mathrm{f}_{2}$ values.

$$
M_{t}=C_{0} V_{0}\left(1-e^{-\frac{K S}{V_{0} P} t}\right)
$$

The in vitro release studies were carried out at $37 \pm 0.5^{\circ} \mathrm{C}$ using the CIB model and the dissolution medium of $10 \%(\mathrm{v} / \mathrm{v})$ iso-propyl alcohol (IPA) in PBS at $\mathrm{pH} 7.4$ was used in this study. The distance between the top of the basket and the bottom of paddle was set at $2 \mathrm{~cm}$. The loading amount of the formulation was about $210 \pm 5 \mathrm{mg}$. The volume of dissolution medium is $900 \mathrm{~mL}$. Other parameters, such as cylinder \# and paddle speed, varied in studies for different purposes and will be specified in the each section.

\subsection{Effect of different surface area (S)}

In order to verify the effect of different surface area (S) in the equation, TBC formulation 3-1c was tested in this study and cylinder \#2 (cylinder diameter $15.0 \mathrm{~mm}$ ) was used in the initial in vitro dissolution study to calculate K. From this calculated K, the amount of drug release from cylinder \#1 and \#3 with different $\mathrm{S}$ could be predicted. The predicted drug release data from calculated $\mathrm{K}$ was compared to the experimental data generated from cylinder \#1 (cylinder diameter $12.5 \mathrm{~mm}$ ) and \#3 (cylinder diameter 17.5 $\mathrm{mm}$ ). The paddle speed was set at $100 \mathrm{rpm}$. The drug concentration at different time points was detected by HPLC method.

By plotting $\operatorname{Ln}\left(\mathrm{C}_{0} \mathrm{~V}_{0}\right)-\operatorname{Ln}\left(\mathrm{C}_{0} \mathrm{~V}_{0}-\mathrm{M}_{\mathrm{t}}\right)$ vs. time (Figure 3-6a), the slope $\mathrm{K}_{\text {app }}$ is 0.0463 day $^{-1}$. The measure $\mathrm{P}$ between TBC and dissolution medium is 4549 . So calculated $\mathrm{K}$ is $0.64 \mathrm{~cm} \cdot \mathrm{hr}^{-1}$. Cylinder \#1 and \#3 were used to test real time drug release and compared to the predicted drug release data (Figure 3-6b1 and 3-6b2). In terms of percentage release, the dissimilarity/similarity factor $\mathrm{f} 1$ and $\mathrm{f} 2$ values were $11.3 / 95.3$ for cylinder \#1 and 7.2/92.8 for cylinder \#3. The results indicate that the Equation 3-9 can be applied to predict the drug release from tested oily vehicle by changing the cylinder with different diameter.

\subsection{Effect of changing several parameters simultaneously}

Equation is verified only when the all the parameters in it can be challenged. In order to test the effect of changing several parameters simultaneously to the Equation 3-9, formulation 3-1a with the concentration of $3.2 \mathrm{mg} / \mathrm{mL}(0.3 \%, \mathrm{w} / \mathrm{w})$ was tested in the initial dissolution study to calculate $\mathrm{K}$. The loading amount was about $210 \pm 5 \mathrm{mg}$ and the cylinder $\# 5$ with the diameter $15 \mathrm{~mm}$ was used. The drug concentration at different 


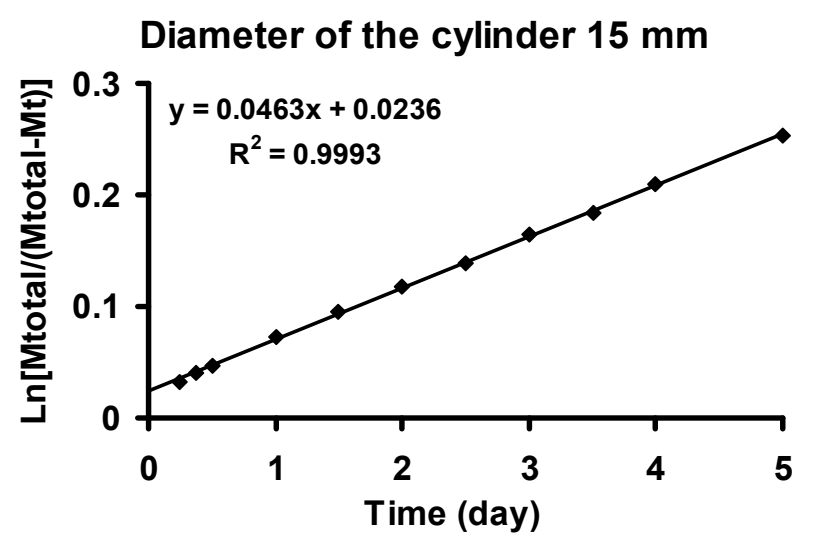

(a)

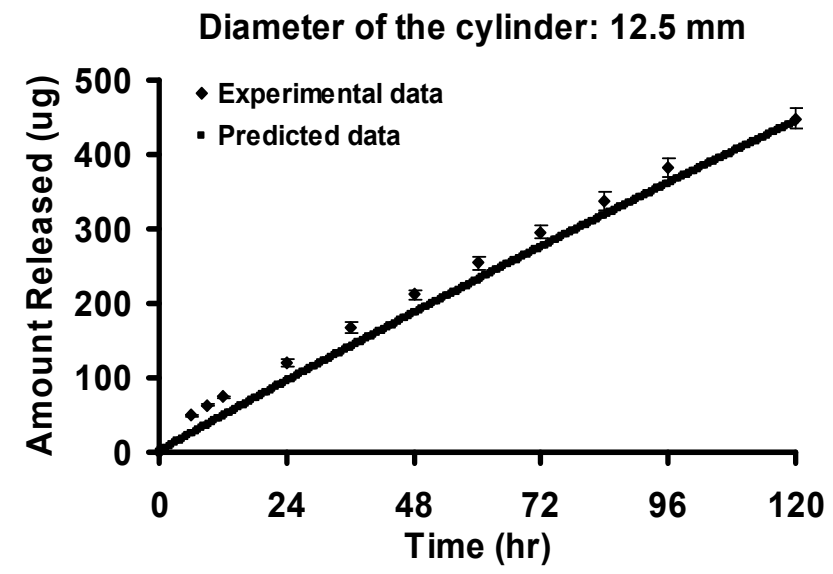

(b1)

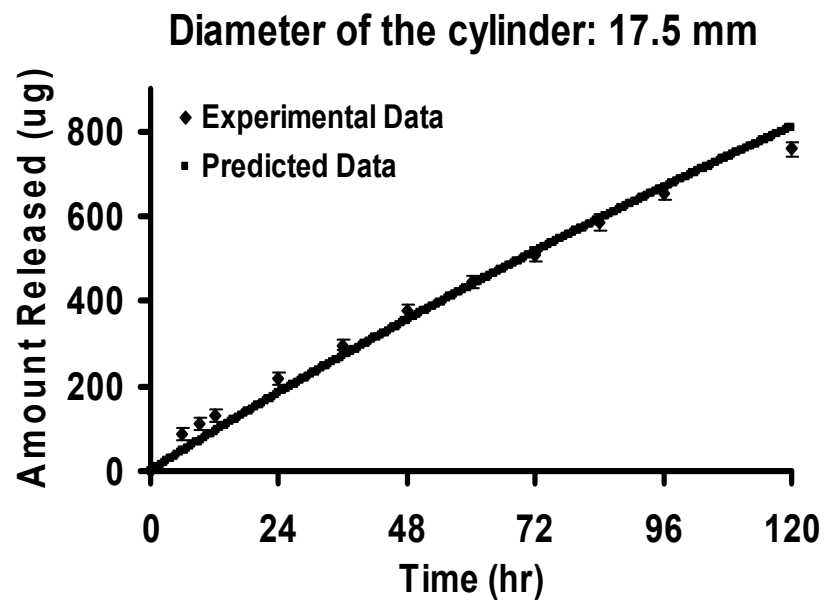

(b2)

Figure 3-6. (a) The release profile of buprenorphine from formulation 3-1c, and predicted data and experimental data generated from (b1) cylinder \#1 and (b2) \#3. 
time points was detected by HPLC method. By plotting $\operatorname{Ln}\left(\mathrm{C}_{0} \mathrm{~V}_{0}\right)-\operatorname{Ln}\left(\mathrm{C}_{0} \mathrm{~V}_{0}-\mathrm{M}_{\mathrm{t}}\right)$ vs. time (Figure 3-7a), the slope $\mathrm{K}_{\mathrm{app}}$ is 0.056 day $^{-1}$. The measured $\mathrm{P}$ is 4549 between TBC and dissolution medium. The calculated $\mathrm{K}$ is $1.24 \mathrm{~cm} \cdot \mathrm{hr}^{-1}$. From the calculated $\mathrm{K}$, the amount drug release was predicted using the Equation 3-9 by changing the parameter(s) of $\mathrm{C}_{0}$, $\mathrm{V}_{0}, \mathrm{C}_{0} \& \mathrm{~V}_{0}, \mathrm{C}_{0} \& \mathrm{~S}$, and $\mathrm{C}_{0}, \mathrm{~V}_{0} \& \mathrm{~S}$, simultaneously.

The effect of $\mathrm{C}_{0}$ was explored by predicting the drug release from the formulations 3-1c and 3-1d with different concentration (1.5\% and 3\%). Figure 3-7b1 and 3-7b2 showed the predicted drug release amount by time and the experimental data. In terms of percentage release, the f2/f1 values were $83.5 / 14$ and $86.9 / 11.9$ for formulation 3-1c and 3-1d, respectively.

The effect of $\mathrm{C}_{0}$ and $\mathrm{V}_{0}$ simultaneously was explored by predicting the drug release from the formulation $3-1 \mathrm{~d}$ with the drug concentration $(3 \%)$ and loading volume $(\sim 0.27 \mathrm{~mL})$. Figure 3-7c showed the predicted drug release amount by time and the experimental data. In terms of percentage release, the f2/f1 values were 86.8/18.2.

Figure 3-7b2 and 3-7c also showed the effect of $\mathrm{V}_{0}(\sim 0.2$ and $0.27 \mathrm{~mL}$, respectively) with the constant drug concentration (3\%) and fixed surface area.

The effect of $\mathrm{C}_{0}$ and $\mathrm{S}$ simultaneously was explored by predicting the drug release from the formulation 3-1c with different drug concentration (1.5\%) in cylinder \#6 with the diameter of $17.5 \mathrm{~mm}$. Figure 3-7d showed the predicted drug release amount by time and the experimental data. In terms of percentage release, the $\mathrm{f} 2 / \mathrm{fl}$ values were 88.5/7.2.

The effect of $\mathrm{C}_{0}, \mathrm{~V}_{0}$ and $\mathrm{S}$ simultaneously was explored by predicting the drug release from the formulation $3-1 \mathrm{c}$ with the drug concentration $(1.5 \%)$, loading volume $(0.26 \mathrm{~mL})$, and the cylinder \#6 with the different diameter of $17.5 \mathrm{~mm}$. Figure 3-7e showed the predicted drug release amount by time and the experimental data. In terms of percentage release, the $\mathrm{f} 2 / \mathrm{f} 1$ values were $88.9 / 8.8$.

According to the FDA guidance, ${ }^{111}$ two dissolution profiles are declared equivalence if $f_{1}$ values are between 0 and 15 and $f_{2}$ value are between 50 and 100 . From aforementioned results, after changing several parameters simultaneously, there is no significant difference between experimental and predicted data. Therefore Equation 3-9 can give a good prediction of the amount of drug release for the tested formulation and it is expected that Equation 3-9 can be used to describe the drug release process in CIB dissolution system.

\subsubsection{IVIVC}

Level A IVIVC represents a point-to-point relationship between in vitro dissolution and the in vivo drug absorption from the tested dosage form. Although there is the lack of the regulatory IVIVC guidance for parenterals, it is believed that the FDA

principles of the IVIVC for modified solid dosage form can be used for parenterals. ${ }^{107}$ 


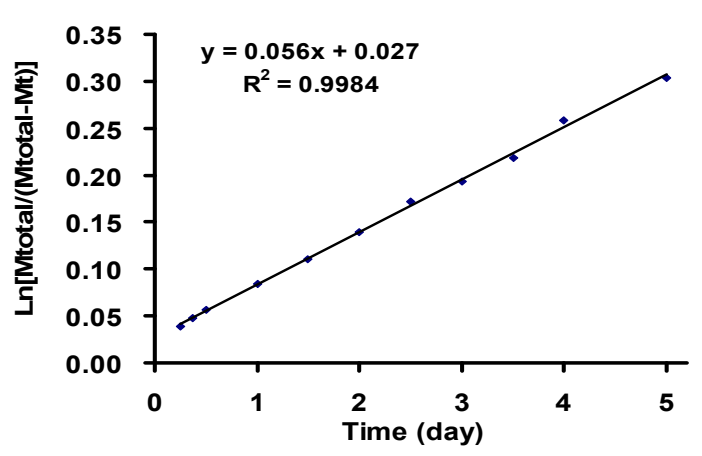

(a)

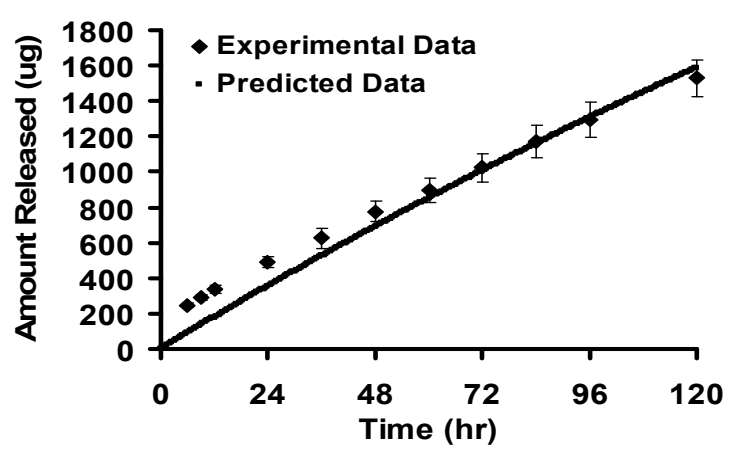

(b.2)

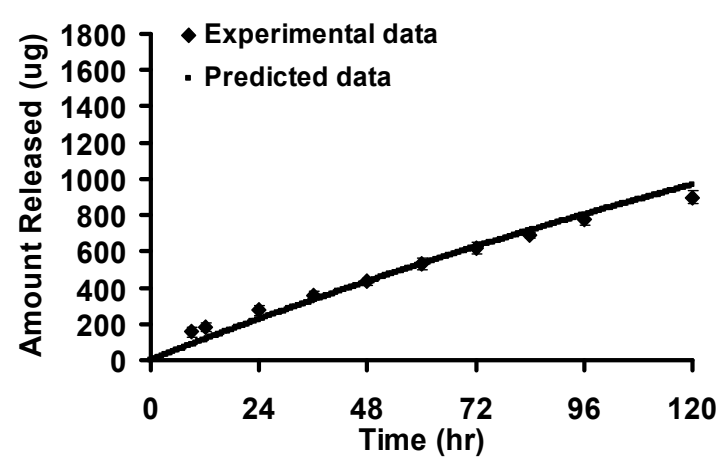

(d)

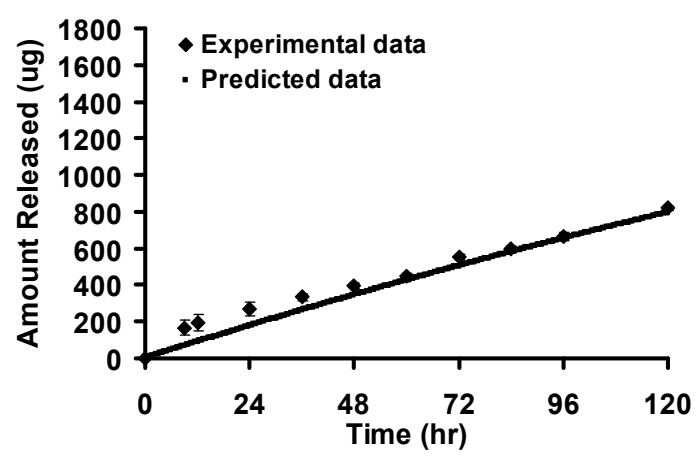

(b1)

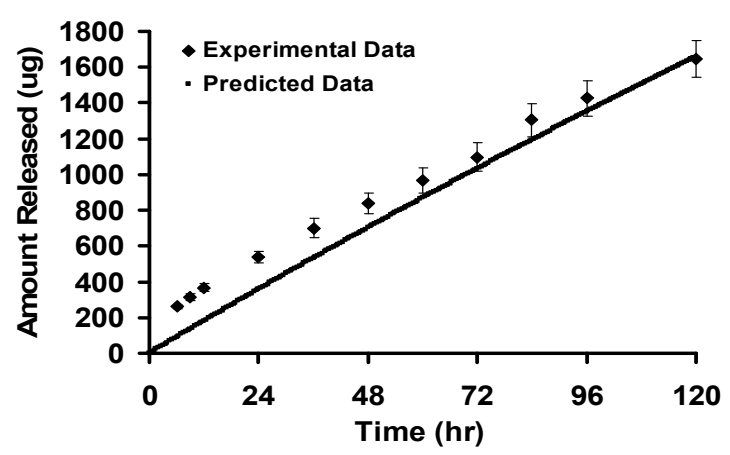

(c)

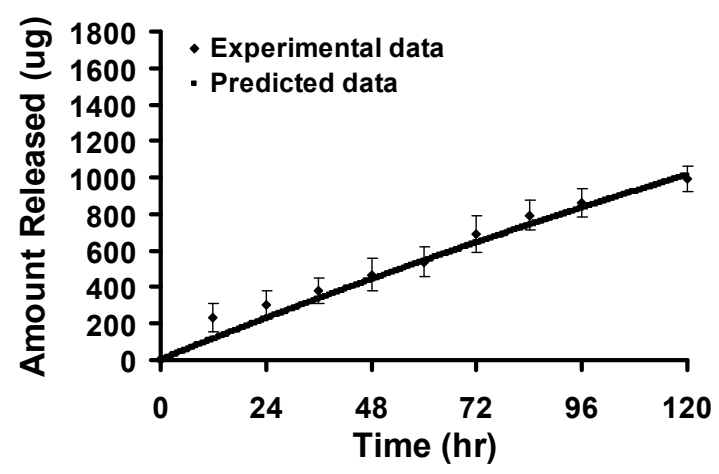

(e)

Figure 3-7. The buprenorphine release profile and the comparison of experimental data and predicted data at different conditions.

(a) The initial buprenorphine release profile from formulation 3-1a with concentration of $0.3 \%$ and loading volume of $\sim 0.2 \mathrm{~mL}$ in the cylinder \#5 (diameter: $15 \mathrm{~mm}$ ).

(b.1) Changing $\mathrm{C}_{0}$ to $1.5 \%$.

(b.2) Changing $\mathrm{C}_{0}$ to $3 \%$.

(c) Changing $\mathrm{C}_{0}$ to $3 \%$ and $\mathrm{V}_{0}$ to $0.27 \mathrm{~mL}$.

(d) Changing $\mathrm{C}_{0}$ to $1.5 \%$ and using cylinder \#6 (diameter: $17.5 \mathrm{~mm}$ ).

(e) Changing $\mathrm{C}_{0}$ to $1.5 \%, \mathrm{~V}_{0}$ to $0.26 \mathrm{~mL}$ and using cylinder \#6 (diameter: $17.5 \mathrm{~mm}$ ). 
For a reasonable IVIVC, FDA guidance ${ }^{108}$ states that two or more formulations with different release rates should be used to demonstrate the IVIVC relationship and the absolute percentage prediction errors (PE\%) of Cmax and AUC should be less than 15\% for each formulation and the average number are less than $10 \%$. If these criteria are not met, evaluation of external predictability of the IVIVC should be performed.

The in vivo drug release data of formulation 3-1b and 3-2 in rat studies were used to develop Level A IVIVC. After intravenously administration of buprenorphine solution at a dose of $0.05 \mathrm{mg} / \mathrm{kg}$, the average values of $K_{10}, K_{12}, K_{21}, \mathrm{~V}_{1}$, and AUC of six SD rats were $1.21 \mathrm{hr}^{-1}, 0.82 \mathrm{hr}^{-1}, 0.94 \mathrm{hr}^{-1}, 764 \mathrm{~mL}$, and $13.2 \mathrm{hr} \cdot \mathrm{ng} \cdot \mathrm{mL}^{-1}$ respectively. After subcutaneous injection of the buprenorphine saline solution at the same dose, the AUC with the value of $18.4 \mathrm{hr} \cdot \mathrm{ng} \cdot \mathrm{mL}^{-1}$ was calculated. After normalizing by dose, the calculated bioavailability was $116 \%$. The value is more than $100 \%$ and this could be caused by the variance of individual rat. The value $100 \%$ of bioavailability was used in the following calculation. Figure 3-8a shows in vivo drug plasma concentration profile of formulation 3-1b and 3-2 prepared with TBC and ATBC, respectively. The figure indicates that drug release rate is different for the two formulations. The drug release occurred in a controlled manner for two weeks. Buprenorphine solution prepared with TBC was able to achieve average Cmax of $8.5 \mathrm{ng} / \mathrm{mL}$ at 6 hours at the dose of $3 \mathrm{mg} / \mathrm{kg}$ and maintain the average plasma concentrations above $0.96 \mathrm{ng} / \mathrm{mL}$ for 336 hours $(14$ days). Buprenorphine solution prepared with ATBC was able to achieve average Cmax of $11.15 \mathrm{ng} / \mathrm{mL}$ at 12 hours at the same dose and maintain the average plasma concentrations above $1 \mathrm{ng} / \mathrm{mL}$ for at least 192 hours ( 8 days). Also the drug release rate of Formulation 3-2 was higher than that of Formulation 3-1b.

The IV plasma profile showed the characters of the distribution and elimination phases, Therefore the Loo-Reigelman method developed from two compartment model was used to calculate the fraction of drug absorbed in vivo (Fa). Figure 3-8b shows the in vivo cumulative drug releases after subcutaneous injection of formulation $3-1 \mathrm{~b}$ and 3-2. The ATBC and TBC formulation displayed different absorption rate. The drug release from $\mathrm{ATBC}$ formulation was faster than that from $\mathrm{TBC}$ formulation. Figure 3-8c indicates a good linear correlation between $\operatorname{Ln}\left[\mathrm{C}_{0} \mathrm{~V}_{0} /\left(\mathrm{C}_{0} \mathrm{~V}_{0}-\mathrm{M}_{\mathrm{t}}\right)\right]$ verse time with $\mathrm{R}^{2}=$ 0.9943 for TBC formulation and 0.9979 for ATBC formulation, respectively. It can be speculated that Equation 3-8 also describes the in vivo drug release after subcutaneous injection of formulation 3-1b and 3-2.

In vitro dissolution study was conducted with the same formulations (Figure 3-9a). The loading volume of the oily formulation was the same with that in in vivo study. The principles of media selection will be discussed in Chapter 4. Since the real time release of tested formulations will last for two weeks, accelerated conditions were applied in dissolution study with the time scale factor of 2 . The dissolution medium of $15 \%$ of n- propyl alcohol in PBS (v/v) with $0.15 \%$ SDS at $\mathrm{pH} 6.0$ was selected and the paddle speed was set at $100 \mathrm{rpm}$. A good linear correlation between $\mathrm{Ln}\left[\mathrm{C}_{0} \mathrm{~V}_{0} /\left(\mathrm{C}_{0} \mathrm{~V}_{0}-\mathrm{M}_{\mathrm{t}}\right)\right]$ and time was also obtained with $\mathrm{R}^{2}=0.9966$ for $\mathrm{TBC}$ formulation and $\mathrm{R}^{2}=0.9991$ for ATBC formulation, respectively (Figure 3-9b). The slopes of the in vivo data were determined to be about $0.0117 \mathrm{hr}^{-1}$ and $0.00718 \mathrm{hr}^{-1}$ (Figure 3-8c) for the "fast" and 


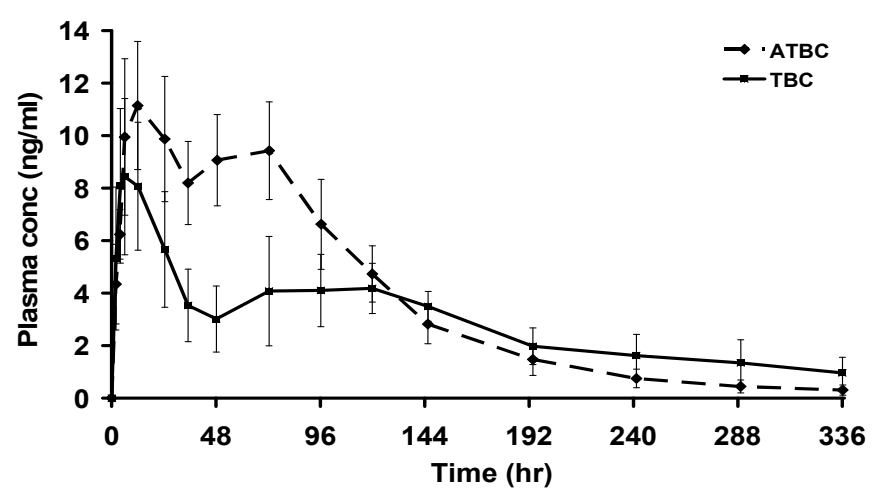

(a)

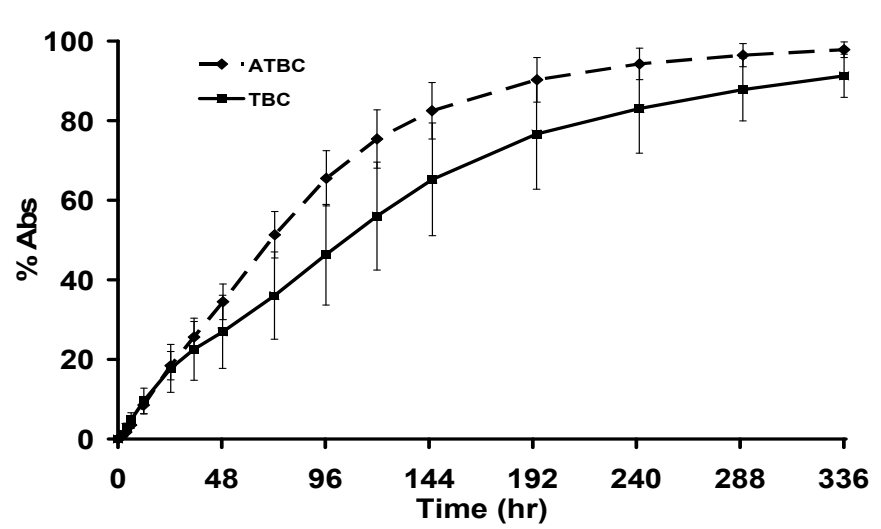

(b)

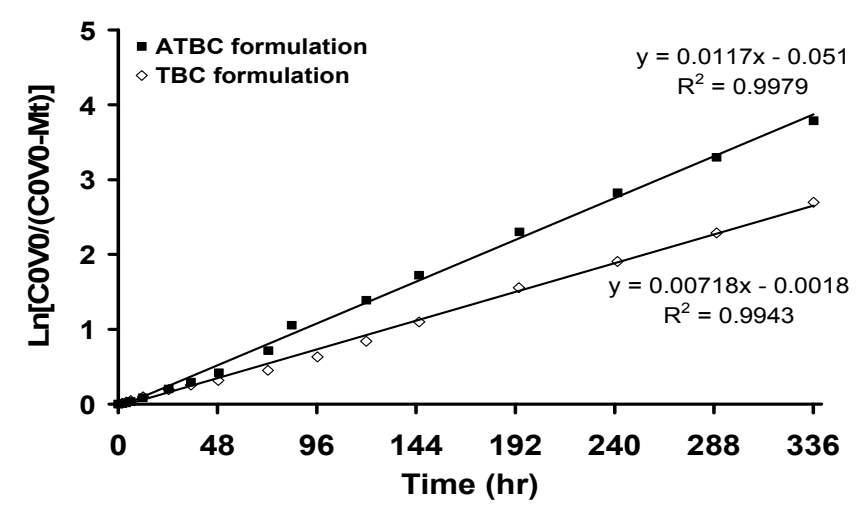

(c)

Figure 3-8. In vivo data process of formulation 3-1b and 3-2 after subcutaneous injection in rats for IVIVC development.

(a) Plasma concentration profile of buprenorphine.

(b) In vivo cumulative drug release profile.

(c) Relationship between $\operatorname{Ln}\left[\mathrm{C}_{0} \mathrm{~V}_{0} /\left(\mathrm{C}_{0} \mathrm{~V}_{0}-\mathrm{M}_{\mathrm{t}}\right)\right]$ and time after subcutaneous injection of formulation 3-1b and 3-2 prepared with different oily vehicles in rat study.

Doses of in vivo studies were $3 \mathrm{mg} / \mathrm{Kg}$. 


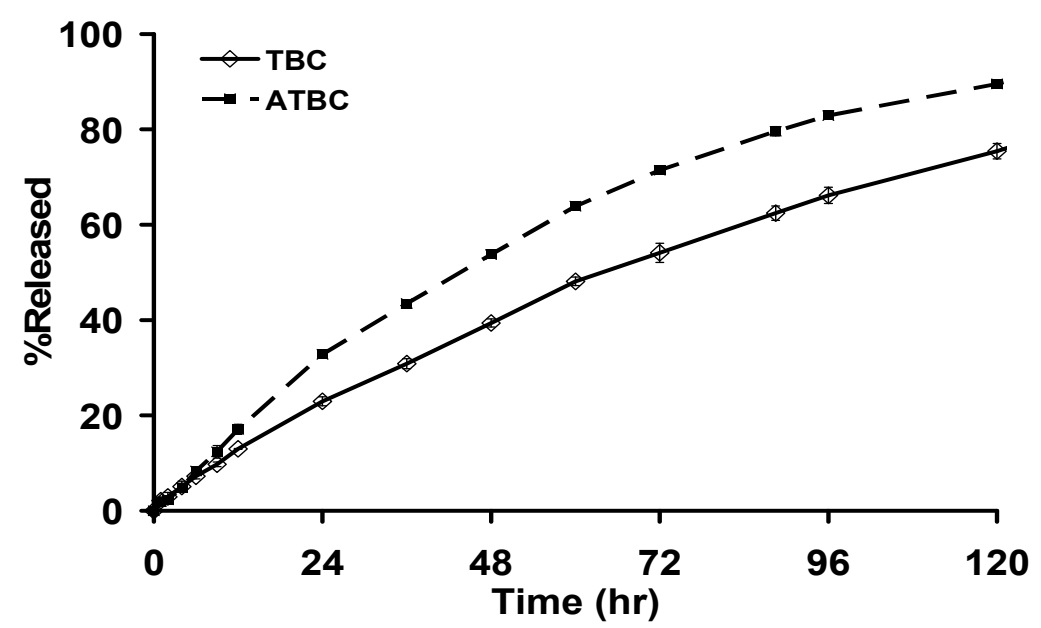

(a)

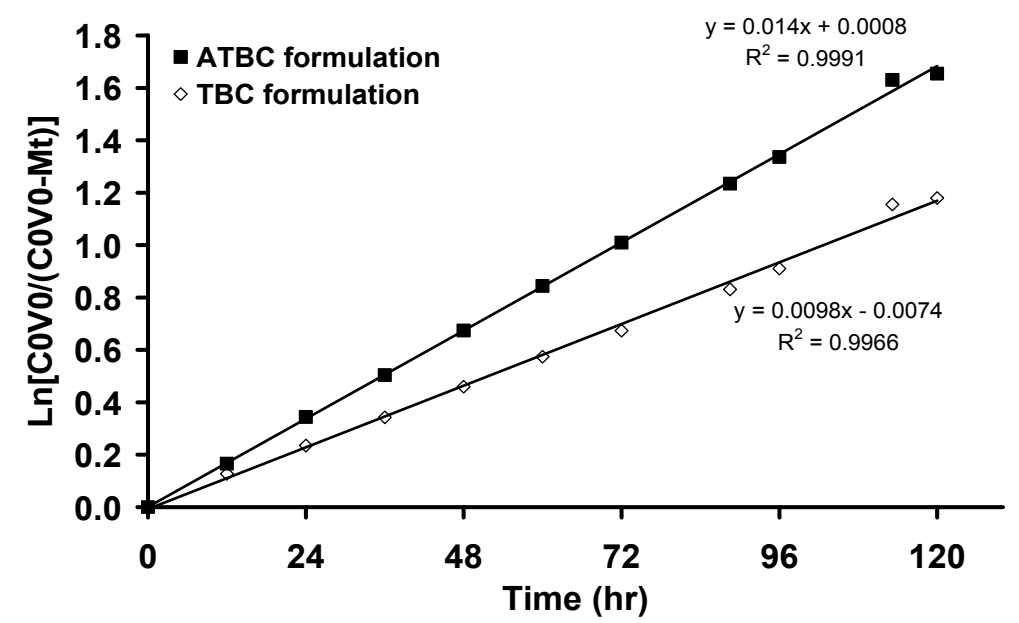

(b)

Figure 3-9. In vitro data process of formulation 3-1b and 3-2 for IVIVC development.

(a) In vitro drug release profiles.

(b) Relationship between $\operatorname{Ln}\left[\mathrm{C}_{0} \mathrm{~V}_{0} /\left(\mathrm{C}_{0} \mathrm{~V}_{0}-\mathrm{M}_{\mathrm{t}}\right)\right]$ and time. 
"slow" release dosage forms, corresponding to the in vitro release slopes of $0.014 \mathrm{hr}^{-1}$ and $0.0098 \mathrm{hr}^{-1}$ (Figure 3-9b) for the relative formulations. These indicated that the mechanism of in vitro drug release mimics those of in vivo drug absorption and there could be a point to point correlation between in vivo and in vitro data.

A level A in-vitro in-vivo correlation was investigated using the percent of drug dissolved and the percent of drug absorbed data for the slow and fast release formulations. A good linear regression relationship was observed between the percent dissolved in the dissolution testing and the percent absorbed for the combined data of the two formulations $(y=1.051 x-0.7036, R 2=0.9918$, Figure 3-10).

The validation of the correlation was performed by determining how well the IVIVC models could predict the rate and extent of buprenorphine absorption as characterized by Cmax and AUC. The IVIVC model predicted drug plasma concentration versus time profiles of formulation 3-1b and 3-2 were compared to the experimental data points. The observed and predicted Cmax, AUC and the percentage errors of internal validation were listed in Table 3-2. The absolute PE\% of Cmax and AUC are less than $15 \%$ for each formulation and the average number are less than $10 \%$, which fit the FDA requirement.

Although it is not required by FDA, formulation 3-3a was used to perform external validation of the IVIVC model. The dissolution medium and conditions used in internal validation were applied to perform in vitro dissolution study. Figure 3-11 shows in vitro drug release profile of formulation 3-3a. It indicates that drug release rate and release profile of formulation 3-3a are different with those of formulation 3-1b and 3-2 (Figure 3-8b). The IVIVC developed from formulations 3-1b and 3-2 was used to predict the plasma concentrations of the new formulation. Table 3-3 indicates the actual/predicted Cmax and AUC are 6.91/7.73 $\mathrm{ng} \mathrm{mL}^{-1}$ and $1138.6 / 1151.4 \mathrm{hr} \bullet \mathrm{ng} \mathrm{mL}^{-1}$, respectively. The absolute $\mathrm{PE} \%$ of Cmax and AUC are less than 15\%. The fraction of drug absorbed in vivo was also calculated by Loo-Reigelman method (Figure 3-12). In this case, the fraction of drug absorb in vivo and percentage of drug released in vitro also showed a good linear relationship (Figure 3-13, $\left.\mathrm{R}^{2}=0.9922\right)$.

\subsection{Conclusions}

To date it is still a challenge to develop a Level A IVIVC for parenteral products and there is no standard in vitro dissolution model with the character of Level A IVIVC for oily formulations. The cylinder-in-basket (CIB) model was designed based on the drug absorption mechanism after subcutaneous injection. A mathematical equation, Equation 3-9, was derived to improve the understanding of the diffusion controlled drug release mechanism involved in the CIB model. The equation was verified by challenging the parameters in the equation and comparing the experimental drug release data with predicted data. According to the equation, in vitro drug release follows the $1^{\text {st }}$ order, and the equation could also describe the in vivo drug release mechanism after subcutaneous 


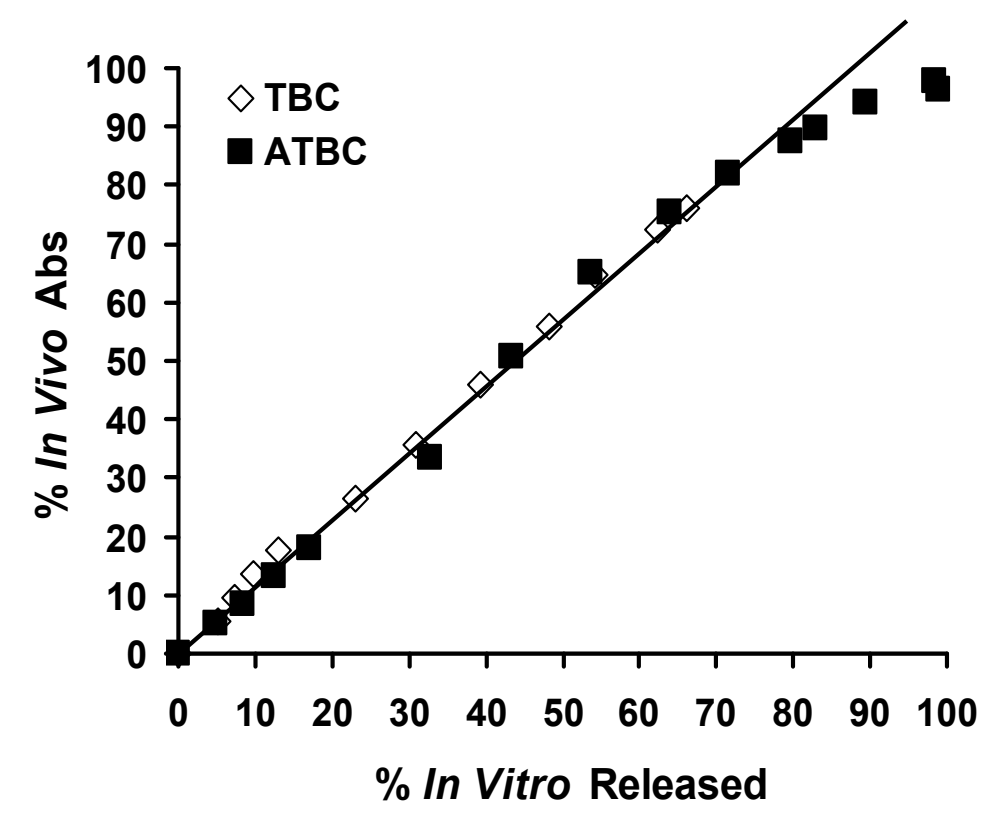

Figure 3-10. IVIVC model linear regression plots of percent absorbed versus percent released for formulation 3-1b and 3-2.

$\% \mathrm{Abs}=1.051 \cdot \%$ Released $-0.7036, \mathrm{R}^{2}=0.9918$. 
Table 3-2. Internal predictability of the IVIVC model.

\begin{tabular}{ccccccccc}
\hline \multirow{2}{*}{ Vehicle } & \multirow{2}{*}{ Formulation } & \multicolumn{3}{c}{ Cmax (ng/mL) } & & \multicolumn{3}{c}{ AUC (hr*ng/mL) } \\
\cline { 3 - 4 } \cline { 7 - 8 } & & Observed & Predicted & PE(\%) & & Observed & Predicted & PE(\%) \\
\hline \multirow{2}{*}{ TBC } & $3-1 \mathrm{~b}$ & 8.44 & 8.85 & 4.9 & & 1179.2 & 1189.84 & 0.9 \\
ATBC & $3-2$ & 11.10 & 9.70 & -12.6 & & 1307.2 & 1262.50 & -3.4 \\
\hline
\end{tabular}

Table 3-3. External predictability of the IVIVC model.

\begin{tabular}{|c|c|c|c|c|c|c|c|}
\hline \multirow{2}{*}{ Vehicle } & \multirow{2}{*}{ Formulation } & \multicolumn{3}{|c|}{$\operatorname{Cmax}(n g / m L)$} & \multicolumn{3}{|c|}{$\operatorname{AUC}\left(h r^{*} n g / m L\right)$} \\
\hline & & Observed & Predicted & PE(\%) & Observed & Predicted & PE(\%) \\
\hline $\begin{array}{c}\text { TEC:ATEC:TBC } \\
(1: 1: 1, \mathrm{v} / \mathrm{v} / \mathrm{v})\end{array}$ & $3-3 a$ & 6.91 & 7.73 & 11.87 & 1138.6 & 1151.425 & 1.1 \\
\hline
\end{tabular}




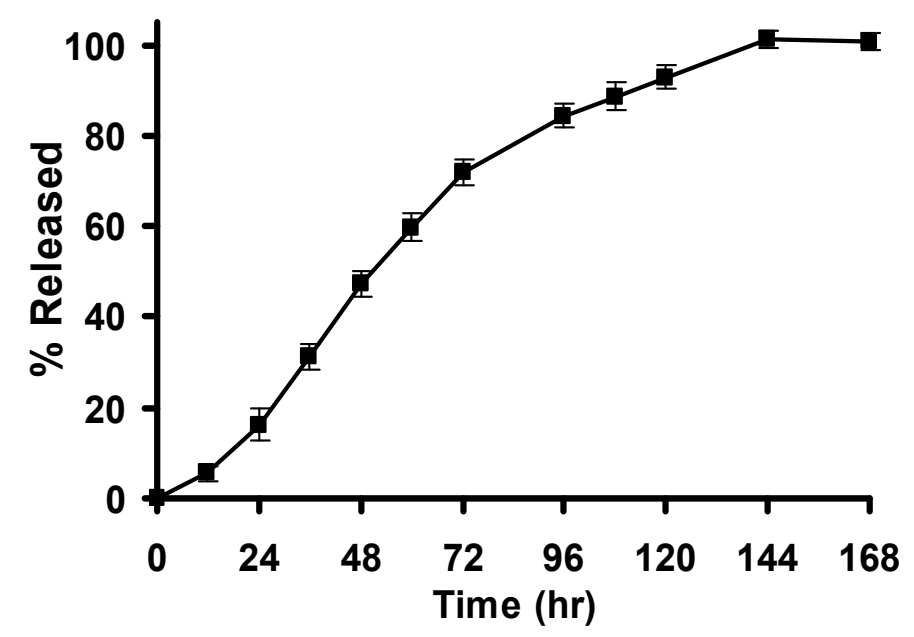

Figure 3-11. In vitro drug release of formulation 3-3a.

$\mathrm{n}=3$.

Data are presented as mean $\pm \mathrm{SD}$. 


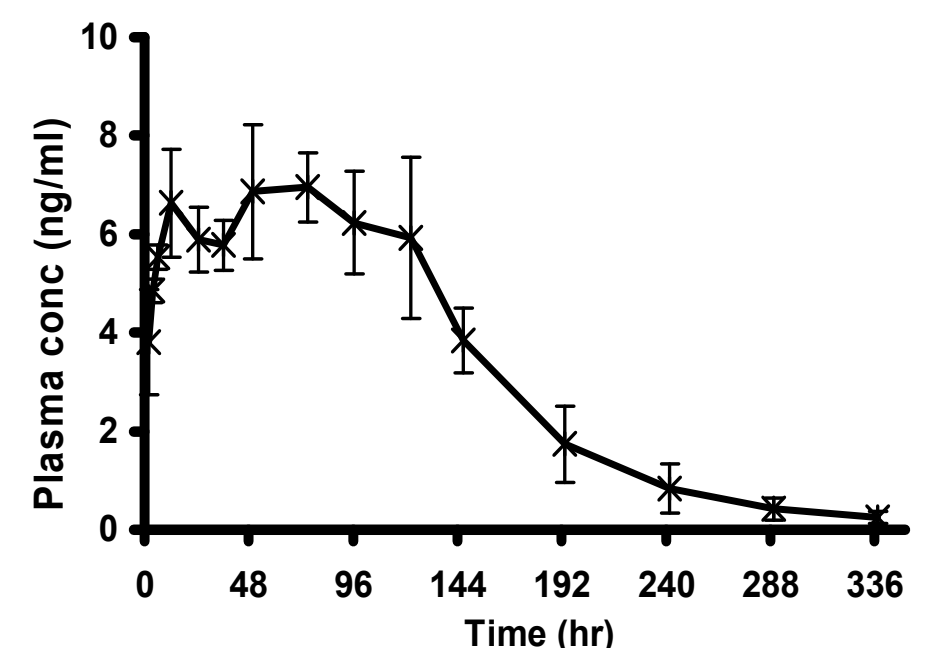

(a)

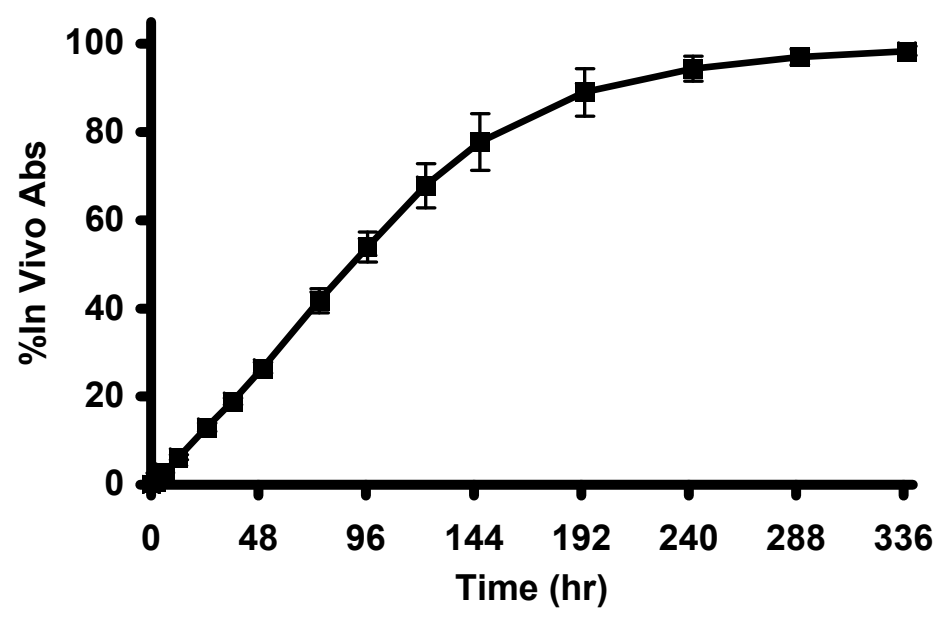

(b)

Figure 3-12. (a) Plasma concentration profile of buprenorphine, and (b) in vivo cumulative drug release profile after s.c. injection of formulation 3-3a in rats.

$\mathrm{n}=4$.

Data are presented as mean $\pm \mathrm{SD}$.

Dose of in vivo study is $3 \mathrm{mg} / \mathrm{Kg}$. 


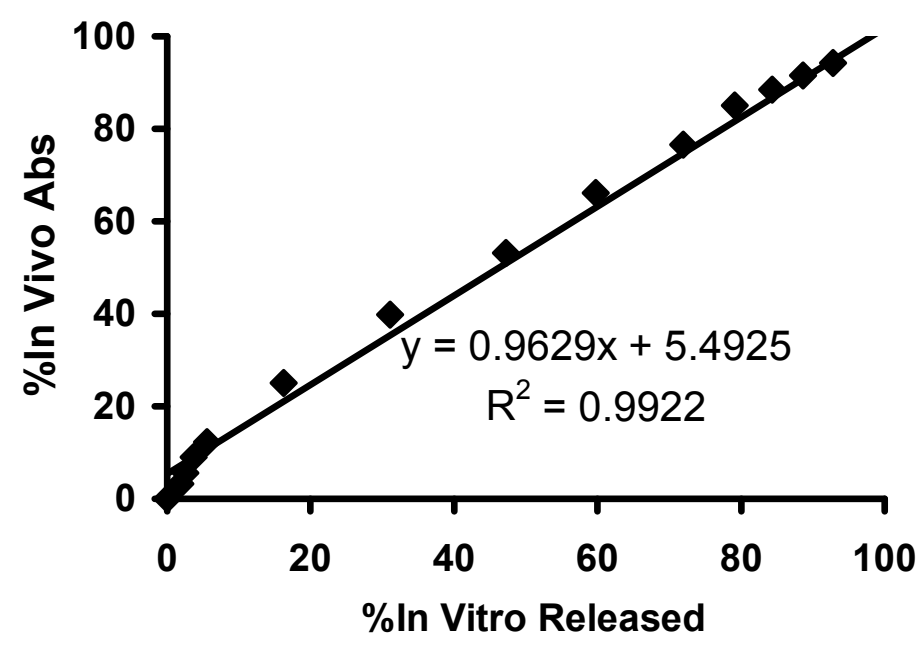

Figure 3-13. Linear regression of percent absorbed versus percent released for formulation 3-3a. 
injection in rat studies. The results of linear relationship between $\mathrm{Fa}$ and $\mathrm{F}_{\text {diss }}$ and validation results suggested that Level A IVIVC was developed for the tested oily formations using the CIB model. This is the first time there is an in vitro dissolution system with the character of level A IVIVC for oily formulations. It also has the potential to be applied to other parenteral dosage forms if they share the same drug release mechanism. The Equation 3-9 was derived from diffusion controlled drug release mechanism and some assumptions were made during the derivation. Therefore all these should be considered if CIB dissolution model was applied to other dosage forms. In addition, CIB devices were modified based on the USP apparatus I \& II. Therefore it is easy to be applied in the dissolution labs since USP apparatus I \& II are the most popular dissolution devices and available in the labs. This novel in vitro dissolution model may contribute as a valuable tool for formulation development, bioequivalence testing, and as an aid in setting dissolution specifications in QC of parenteral products. 


\section{CHAPTER 4. APPLICATION OF A NOVEL IN VITRO DISSOLUTION MODEL: A RATIONAL METHOD TO SELECT THE DISSOLUTION CONDITIONS IN AN ACCELERATED DISSOLUTION STUDY}

\subsection{Introduction}

Unlike extended release (ER) solid dosage forms, ER parenterals were usually designed to release drug for days, weeks, months, and even years. The real-time dissolution analysis of ER parenteral formulations is time-consuming and sometimes impractical. Accelerated in vitro release study can correlate short-time release data with real-time release data in order to predict real-time drug release and minimize the potential problems in the real-time release studies, such as stability. Therefore, accelerated in vitro release testing methods are important and helpful for quality control of the product and ER parenteral formulation development.

Usually the problem for accelerated study is that accelerated methods may not be bio-relevant and often change not only the drug release rate but also the mechanism of release. $^{70,103}$ This change may not affect quality control and formulation development of the ER parenterals, but it could increase the difficulty to establish Level A in vitro-in vivo correction (IVIVC). In order to increase drug release rate without changing the release mechanism in the accelerated study, it is very important to choose suitable in vitro dissolution model and conditions, and understand how the variables affect the drug release mechanism.

Temperature, solvent, $\mathrm{pH}$, surfactants, agitation rate, etc ${ }^{106,114}$ have been successfully applied in the accelerated study to change the drug release rate, but it is always a challenge, especially for the ER parenterals, to explore the rational method to find the desired dissolution conditions for in vitro accelerated testing in a short-time period. Ideally, the accelerated in vitro study should be bio-relevant and correlate with the in vivo study, especially if the accelerated method is to be used as a surrogate for in vivo studies. ${ }^{70}$ In addition, the ideal accelerated condition should only increase the drug release rate without changing the release mechanism. The Cylinder-in-Basket (CIB) method has shown bio-relevant with the character of level A IVIVC and discussed in Chapter 3. The drug release mechanism of CIB model follows the first-order kinetic and Equation 4-1 is developed to describe in vitro drug release in this model. Equation 4-2 is derived from Equation 4-1 and is used to describe the in vivo drug release of tested formulations after subcutaneous injection.

For in vitro:

$$
\begin{aligned}
& \operatorname{Ln}\left(C_{0} V_{0}\right)-\operatorname{Ln}\left(C_{0} V_{0}-M_{t}\right)=K_{a p p} t \\
& K_{a p p}=\frac{D S}{h V_{0} P}=\frac{K S}{V_{0} P}
\end{aligned}
$$


For in vivo:

$$
\operatorname{Ln} M_{\text {total }}-\operatorname{Ln}\left(M_{\text {total }}-M_{t}\right)=K_{a p p} t
$$

where $\mathrm{C}_{0}$ and $\mathrm{V}_{0}$ represent initial drug concentration and volume of loaded formulation; $\mathrm{M}_{\mathrm{t}}$ represents the amount of drug at different time in aqueous phase in in vitro studies and the amount of drug released at different time point in in vivo studies; $\mathrm{D}, \mathrm{h}$ and $\mathrm{S}$ represent diffusion coefficient, the length of diffusion layer, and oil-water interface area; the $\mathrm{K}$ is the ratio of $\mathrm{D}$ divided by $\mathrm{h}$ and $\mathrm{K}_{\mathrm{app}}$ is the apparent rate constant; $\mathrm{M}_{\text {total }}$ represent the total amount of drug released at different time point in in vivo studies.

Theoretically, drug release rate, $\mathrm{K}_{\mathrm{app}}$, should be altered without changing the release mechanism. $\mathrm{K}_{\text {app }}$ is related to $\mathrm{K}, \mathrm{S}, \mathrm{V}_{0}$ and $\mathrm{P}$. Therefore it is possible to find a rational method to select dissolution medium based on the information of $\mathrm{K}_{\text {app. }}$. The purpose of this study is to apply CIB dissolution model in the accelerated study for parenterals and develop a rational method to select the accelerated dissolution condition in a short time period.

\subsection{Materials and Methods}

\subsubsection{Materials}

Buprenorphine base was obtained from Diosynth Inc. (Morrisville, NC). Tributyl citrate (TBC), and acetyltributyl citrate (ATBC) were obtained from Morflex, Inc. (Greensboro, NC).

\subsubsection{Analytical methodology - HPLC/Fluorescence analyses}

Validated HPLC method developed to quantitate buprenorphine in the samples obtained from in vitro studies was described in Chapter 2. Briefly, Discovery C-18 column $(2.1 \times 100 \mathrm{~mm})$ (Supelco, Bellefonte, PA) was used. The mobile phase was composed of a mixture of $51 \mathrm{mM} \mathrm{KH}_{2} \mathrm{PO}_{4}$ buffer and acetonitrile $(\mathrm{ACN})(45: 55, \mathrm{v} / \mathrm{v})$ with the final $\mathrm{pH} 6.65$ adjusted by $10 \mathrm{~N} \mathrm{NaOH}$ solution. The oven temperature was $40^{\circ} \mathrm{C}$ and the flow rate was $0.3 \mathrm{~mL} / \mathrm{min}$. The total run time was 6 minutes. Buprenorphine was detected with fluorescence detector at $\lambda_{\text {Exitation }}=292 \mathrm{~nm}$ and $\lambda_{\text {Emission }}=350 \mathrm{~nm}$.

\subsubsection{Dissolution method}

In vitro dissolution studies of buprenorphine ER formulations were tested in the cylinder-in-basket (CIB) dissolution model. ${ }^{115}$ The oily formulation was loaded into the cavity of the cylinder with the diameter of $15 \mathrm{~mm}$ and the height of $24.6 \mathrm{~mm}$. After covering with the 40-mesh United States Pharmacopeia (USP) basket, the cylinder-in- 
basket kit was transferred to 1-Liter vessel. The $900 \mathrm{~mL}$ dissolution medium was carefully loaded into the vessel at the rate of $7 \mathrm{~mL} / \mathrm{sec}$ by modified Hanson Media Mate (Hanson Research Corp.). Different dissolution media were tested for different purposes and the content of the media will be specified in the following discussion section. The USP paddle was then loaded on the top of the basket with the distance of $2 \mathrm{~cm}$ between paddle and basket. The vessels were covered with a lid and tightened with laboratory para-film in order to avoid evaporation of the medium. The experiments were conducted in triplet. During the experiment, $1 \mathrm{~mL}$ of samples was withdrawn from bulk dissolution medium at predetermined time points by AutoPlus Autosampler (Hanson Research Corp.). After sampling, $1 \mathrm{~mL}$ blank dissolution medium was refilled into the bulk dissolution medium. Drug content in the samples was measured by HPLC method. The stability studies were also performed in triplet and conducted at $25 \pm 0.5^{\circ} \mathrm{C}$ and $40 \pm 0.5$ ${ }^{\circ} \mathrm{C}$ ovens for tested formulations in all tested dissolution media. The results showed that buprenorphine was stable in the all dissolution media at $40 \pm 0.5^{\circ} \mathrm{C}$ for the length of tested dissolution time.

The amount of drug release in percentage from the oily solutions was calculated according to Equation 4-2.

$$
\% \text { released }=\frac{V_{s} \cdot \sum_{n=1}^{n} C_{n-1}+V_{b} \cdot C_{n}}{M_{\text {total }}} \cdot 100 \%
$$

where $V_{\mathrm{s}}$ is the sampling volume $\left(1 \mathrm{~mL}\right.$ in this study) at different time points and $\mathrm{V}_{\mathrm{b}}$ is the volume of total dissolution medium. $C_{n}$ and $C_{n-1}$ represent the drug concentration in sample $n$ and $n-1$. $M_{\text {total }}$ is the total amount of drug in the tested formulation.

\subsubsection{Formulation preparation}

In this study, buprenorphine ER oily formulations were used as the model system and the formulation preparation was described as following. An appropriate amount of buprenorphine powder was accurately weighed and dispersed into an appropriate amount of solvent (Table 4-1) in $20 \mathrm{~mL}$ glass scintillation vials. The subsequent suspension was sonicated under $25^{\circ} \mathrm{C}$ until all drug powder was dissolved. The drug concentration was verified by HPLC method. The formulation 4-1 and 4-2 have the same composition as formulation 3-2 and 3-1b, respectively.

\subsubsection{Partition coefficients}

The partition coefficient testing of buprenorphine between the oily vehicles and dissolution media was performed in triplicate. Solutions of drug in aqueous phasesaturated oily vehicles were allowed to equilibrate with the oil phase-saturated aqueous buffers in the incubator at $100 \mathrm{rpm}$ and $37 \pm 0.5^{\circ} \mathrm{C}$ for at least $48 \mathrm{hr}$. Drug concentrations 
Table 4-1. Formulations tested in the method development of accelerated dissolution study.

\begin{tabular}{ccc}
\hline Formulation & Solvent & $\begin{array}{c}\text { Concentration of Buprenorphine in Solvent } \\
\mathbf{\%}(\mathbf{w} / \mathbf{w})\end{array}$ \\
\hline $4-1$ & ATBC & 0.5 \\
$4-2$ & TBC & 0.5 \\
\hline
\end{tabular}


in the aqueous phase and oily phase were determined by HPLC method. The partition coefficients were calculated according to Equation 3-1.

$$
P=\frac{C_{o i l}}{C_{a q}}
$$

where $C_{o i l}$ and $C_{a q}$ represent the drug concentration in the oil and the aqueous phase at the equilibrium state, respectively.

\subsection{Results and Discussion}

Dissolution temperature and medium are two major conditions altered in the accelerated in vitro study. Dr. Burgess stated that elevated temperature in accelerated release testing was not suitable for PLGA microsphere systems in which release mechanism was diffusion controlled. ${ }^{116}$ It is speculated that elevated temperature may not be suitable for oily formulation in the accelerated study since it shared the same diffusion controlled release mechanism. Therefore one of the common parameters, temperature, was not selected to explore in this accelerated study. The altered $\mathrm{pH}$ and different amount of surfactant in the dissolution medium can accelerate the drug release rate and the effects of these parameters were explored in this study using CIB in vitro dissolution model.

The $\mathrm{pH}$ effect of dissolution medium was firstly explored using USP buffer with $\mathrm{pH} 7.4,6.8,6.0$ and 5.0. The release of buprenorphine from formulation 4-1 and 4-2 in different oily vehicles, ATBC and TBC, was tested. The cylinder with the diameter of 15 $\mathrm{mm}$ and the height of $24.6 \mathrm{~mm}$ was used in this study. Figure 4-1 shows the drug release effect of the dissolution medium with different $\mathrm{pH}$. The partition coefficients between different oily phases and dissolution media were also tested and the results are listed in Table 4-2. Buprenorphine is a weak base. When $\mathrm{pH}$ of the dissolution media changed, the ionization extent of drug will change, therefore solubility of drug in the dissolution media and the partition coefficient between oily phase and media will also vary. When the $\mathrm{pH}$ of dissolution medium changed from 7.4 to 5 , the drug release rate increases and the partition coefficient decreases.

Equation 4-1 described the drug release in the USP phosphate buffer with the $\mathrm{pH}$ of 6, 6.8 and 7.4 and the linear relationship $\left(\mathrm{R}^{2}>0.99\right.$, Table 4-2) was obtained after plotting $\mathrm{Ln}\left[\mathrm{C}_{0} \mathrm{~V}_{0} /\left(\mathrm{C}_{0} \mathrm{~V}_{0}-\mathrm{Mt}\right)\right]$ versus time. This indicated when $\mathrm{pH}$ of the dissolution media changed from 7.4 to 6.0 , the drug release could be described by the same equation and the release mechanism does not change. It followed $1^{\text {st }}$-order release for the tested formulations and could be applied in the accelerated studies since the release rate could be increased without changing the release mechanism. Unfortunately, good linear relationship was not observed when applying $\mathrm{pH}$ 5.0 USP neutralized phthalate buffer $\left(\mathrm{R}^{2}=0.9603\right.$ for formulation $4-1 ; \mathrm{R}^{2}=0.948$ for formulation $\left.4-2\right)$. The reason could be that $\mathrm{pH}$ effect or the drug release behavior in phthalate buffer solution (USP buffer, $\mathrm{pH}$ 5) may be different with that in phosphate solutions (USP buffer, $\mathrm{pH} \mathrm{6,6.8} \mathrm{and} \mathrm{7.4).}$ 
In vitro release of buprenorphine from $\mathrm{ATBC}$ formulaiton

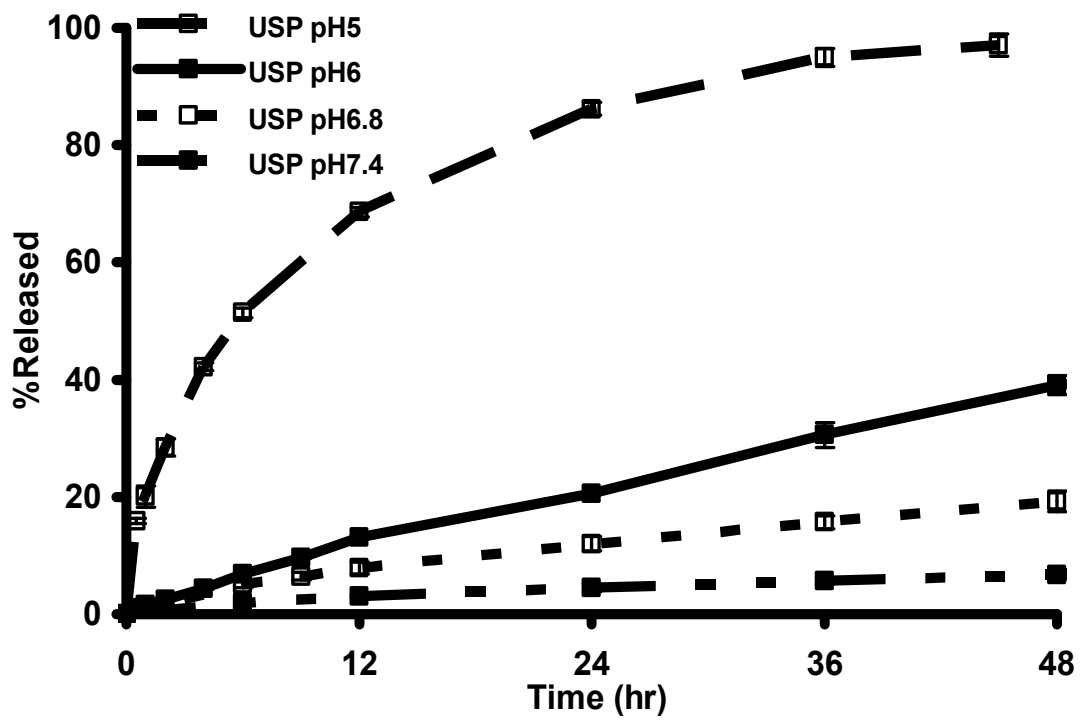

(a)

In vitro release of buprenorphine from TBC formulation

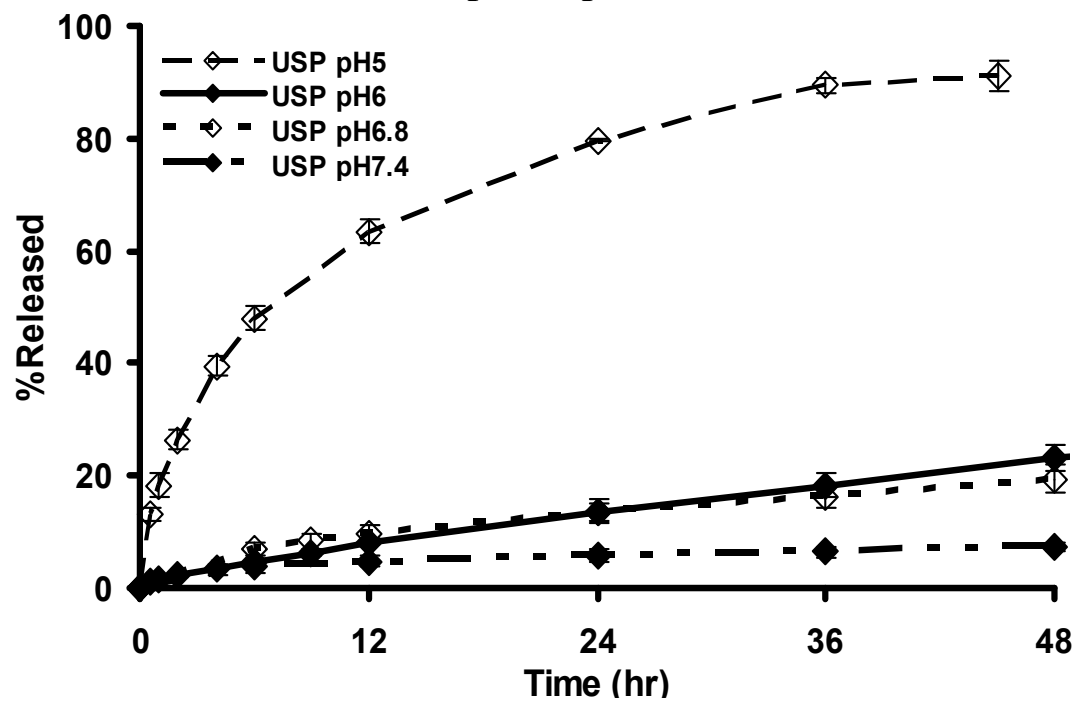

(b)

Figure 4-1. In vitro drug release profiles from (a) formulation 4-1 and (b) formulation 4-2 in USP specified dissolution media at different $\mathrm{pH}$.

$\mathrm{n}=3$.

Data are presented as mean $\pm \mathrm{SD}$. 
Table 4-2. Dissolution paddle speed, partition coefficient and calculated parameters in accelerated studies.

\begin{tabular}{|c|c|c|c|c|c|c|c|c|c|}
\hline \multirow{2}{*}{ Dissolution Medium } & \multirow{2}{*}{$\begin{array}{c}\text { Paddle } \\
\text { Speed } \\
\text { (rpm) }\end{array}$} & \multicolumn{4}{|c|}{$\begin{array}{c}\text { Formulation } 4-1 \\
\text { (Oily Phase: ATBC) }\end{array}$} & \multicolumn{4}{|c|}{$\begin{array}{c}\text { Formulation } 4-2 \\
\text { (Oily Phase: TBC) }\end{array}$} \\
\hline & & $\mathbf{P}$ & $\underset{\left(h r^{-1}\right)}{K_{\text {app }}}$ & $\mathbf{R}^{2}$ & $\begin{array}{c}\mathrm{K} \\
(\mathrm{cm} / \mathrm{hr})\end{array}$ & $\mathbf{P}$ & $\begin{array}{c}K_{\text {app }} \\
\left(\mathbf{h r} \mathbf{r}^{-1}\right)\end{array}$ & $\mathbf{R}^{2}$ & $\begin{array}{c}\mathrm{K} \\
(\mathrm{cm} / \mathrm{hr})\end{array}$ \\
\hline USP buffer $\mathrm{pH} 7.4$ & 50 & $8992.7 \pm 323.1$ & 0.0010 & 0.9951 & 1.094 & $1953.9 \pm 110.7$ & 0.0008 & 0.9937 & 0.1853 \\
\hline USP buffer pH 6.8 & 50 & $2129.1 \pm 75.5$ & 0.0034 & 0.9946 & 0.8806 & $564.5 \pm 3.8$ & 0.0029 & 0.9934 & 0.1941 \\
\hline USP buffer $\mathrm{pH} 6.0$ & 50 & $403.2 \pm 26.9$ & 0.0082 & 0.9913 & 0.4022 & $151.7 \pm 12.7$ & 0.005 & 0.9933 & 0.0773 \\
\hline USP buffer pH 5.0 & 50 & $35.6 \pm 1.6$ & 0.1117 & 0.9603 & NA & $64.8 \pm 2.1$ & 0.0923 & 0.9480 & NA \\
\hline PBS with $0.05 \%(\mathrm{w} / \mathrm{v}) \mathrm{SDS}$ at $\mathrm{pH} 6$ & 50 & $139.6 \pm 29.5$ & 0.0269 & 0.9910 & 0.4501 & $756.5 \pm 35.2$ & 0.0084 & 0.9901 & 0.7525 \\
\hline PBS with $0.1 \%(w / v)$ SDS at $\mathrm{pH} 6$ & 50 & $47.4 \pm 1.6$ & 0.0831 & 0.9938 & 0.4567 & $331.5 \pm 8.5$ & 0.0201 & 0.9997 & 0.769 \\
\hline PBS with $0.8 \%(w / v)$ SDS at $\mathrm{pH} 6$ & 50 & $2.5 \pm 0.1$ & 0.4019 & 0.8557 & NA & $16.1 \pm 0.6$ & 0.4679 & 0.9829 & 0.874 \\
\hline PBS with $0.1 \%(w / v)$ SDS at $\mathrm{pH} 7.4$ & 150 & $233.6 \pm 7.2$ & 0.0485 & 0.9994 & 1.34 & $3979.5 \pm 89.0$ & 0.0119 & 0.9978 & 5.43 \\
\hline PBS with $0.2 \%(\mathrm{w} / \mathrm{v}) \mathrm{SDS}$ at $\mathrm{pH} 7.4$ & 150 & $43.1 \pm 2.3$ & 0.7732 & 0.9904 & 3.83 & $324.6 \pm 5.5$ & 0.0957 & 0.9940 & 3.53 \\
\hline PBS with $0.8 \%(w / v)$ SDS at $\mathrm{pH} 7.4$ & 150 & $28.9 \pm 4.0$ & 1.2097 & 0.9904 & 2.94 & $31.2 \pm 3.3$ & 0.6876 & 0.9997 & 2.45 \\
\hline
\end{tabular}

PBS: phosphate buffer saline solution

NA: not accessed

$\mathrm{K}_{\text {app }}$ and $\mathrm{R}^{2}$ were obtained by plotting $\operatorname{Ln}\left[\mathrm{C}_{0} \mathrm{~V}_{0} /\left(\mathrm{C}_{0} \mathrm{~V}_{0}-\mathrm{M}_{\mathrm{t}}\right)\right]$ versus time and $\mathrm{K}$ was calculated from Equation 4-1.

$\mathrm{n}=3$.

Data of partition coefficient $(\mathrm{P})$ are presented as mean $\pm \mathrm{SD}$. 
The ideal accelerated condition should increase drug release rate without changing the drug release mechanism. According to Equation 4-1, the apparent drug release rate, $\mathrm{K}_{\mathrm{app}}$, is related with the parameters of $\mathrm{K}, \mathrm{S}, \mathrm{V}_{0}$ and $\mathrm{P}$. Amongst four parameters, $\mathrm{S}$ and $\mathrm{V}_{0}$ are considered as the constant for the selected cylinder and loading volume. Diffusion transportation is affected by agitation, medium viscosity and medium temperature. ${ }^{117}$ Agitation could affect the thickness of diffusion layer, h. According to the Stokes-Einstein equation, D is related to solvent viscosity, temperature and solute molecule radius. For the given drug molecule, oily vehicle, dissolution medium, temperature and pressure, the $\mathrm{D}$ could be considered as the constant. The change of dissolution medium would simultaneously change $\mathrm{h}$ and $\mathrm{P}$ between oil phase and media, therefore the $\mathrm{K}$ value will vary. In the accelerated testing, temperature, dissolution medium, and/or agitation speed has to be the parameters used to accelerate the drug release rate. Both values of $\mathrm{K}$ and $\mathrm{P}$ will always vary simultaneously in these situations. It seems impractical to apply Equation 4-1 to select accelerated condition since none of the two variables, $\mathrm{P}$ and $\mathrm{K}$, is the constant for different dissolution media.

But the question is when altered dissolution medium is used to increase dissolution rate, such as $\mathrm{pH}$ or amount of surfactant in the medium, how does the $\mathrm{K}$ value vary? Table 4-2 lists the experimental $\mathrm{K}$ values for tested formulations in the dissolution medium with different $\mathrm{pH}$. When $\mathrm{pH}$ is changed from 7.4 to 6.0 , the $\mathrm{P}$ values decrease 22.3 and 30.2 folds for two formulations, respectively, while the $\mathrm{K}$ values decrease 2.7 and 2.4 folds, respectively. This indicates that the variance of $\mathrm{P}$ is much larger than that of K. Therefore, if the P values have just "slightly difference", the variance of K value could be rounded and considered as a constant. If $\mathrm{K}$ can be considered as the constant, it gives the possibility to select dissolution medium with desired drug release rate just from tested $\mathrm{P}$ values between oily vehicles and dissolution media.

The assumption, "if the $\mathrm{P}$ values have just slightly difference, the variance of $\mathrm{K}$ value could be rounded and considered as a constant", is made here. When it is validated, it will be helpful to choose dissolution medium based on the $\mathrm{P}$ values in the accelerated study. The procedures (Figure 4-2) of selecting dissolution medium in accelerated studies were listed as following.

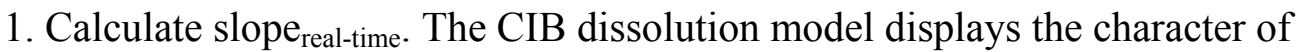
Level A IVIVC for the oily formulations. Therefore the in vivo fraction of drug absorbed $(\mathrm{Fa})$ profiles were applied as the real-time in vitro dissolution release profiles since Level A IVIVC has been established for the tested formulations. ${ }^{115}$ Plot real-time in vivo release profile of long term sustained release oily formulation from in vivo data and calculate slope real-time $_{\text {by }}$ plotting $\mathrm{Ln}\left[\mathrm{M}_{\text {total }} /\left(\mathrm{M}_{\text {total }}-\mathrm{M}_{\mathrm{t}}\right)\right]$ versus time. The value slope $\mathrm{real}_{\text {-time }}$ is calculated from in vivo data and considered as the real-time slope of in vitro release.

2. Select time scale factor.

3. Select the type of cylinder and calculate $S$.

4. Decide the loading volume $\left(\mathrm{V}_{0}\right)$ for in vitro testing. For IVIVC purpose, the recommended loading volume is to keep the same volume used for in vivo study. 


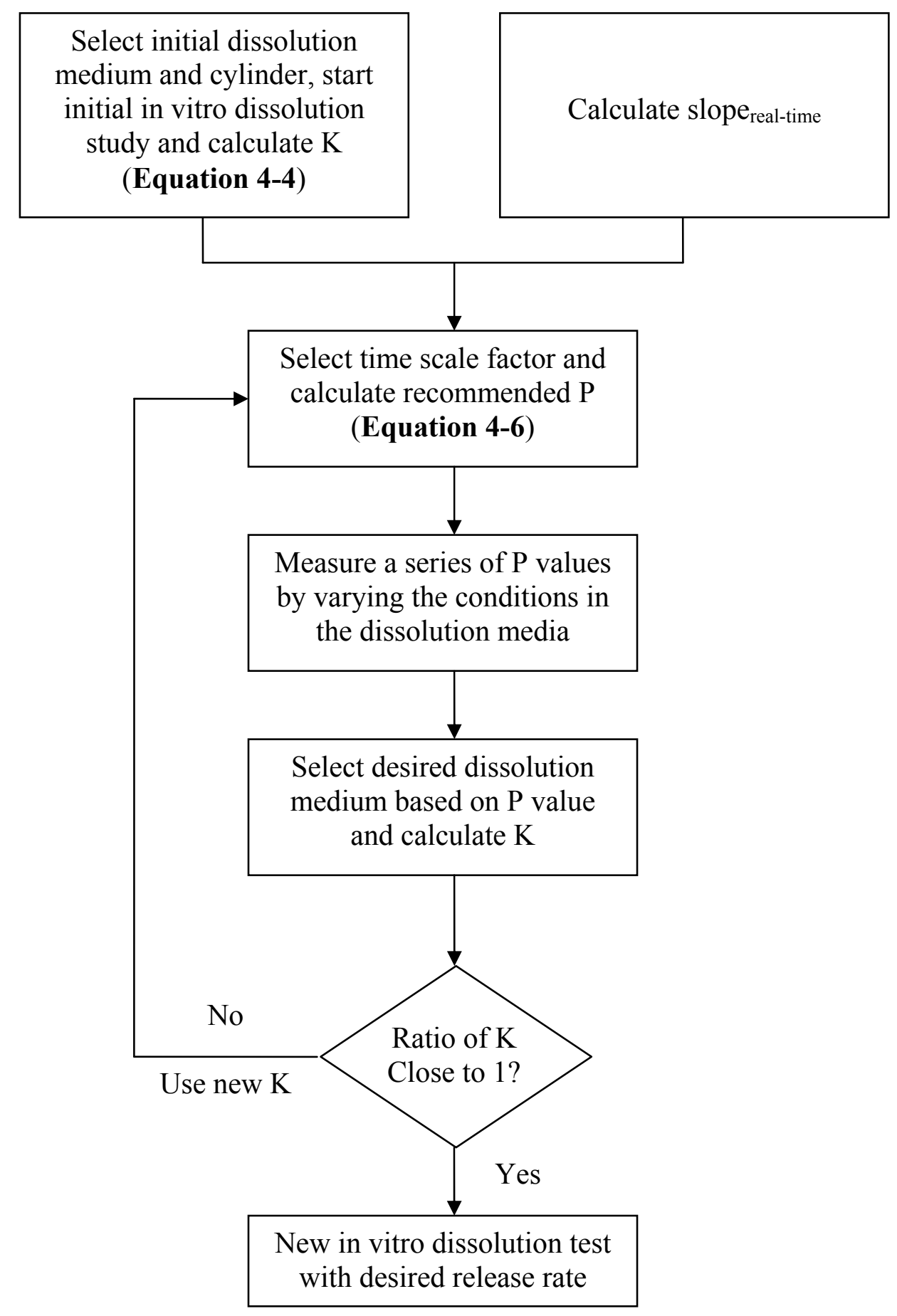

Figure 4-2. The procedures of selecting dissolution medium in accelerated study. 
5. Run the initial in vitro dissolution test using the CIB dissolution model and calculate the $\mathrm{K}$ value by plotting $\mathrm{Ln}\left[\mathrm{C}_{0} \mathrm{~V}_{0} /\left(\mathrm{C}_{0} \mathrm{~V}_{0}-\mathrm{M}_{\mathrm{t}}\right)\right]$ versus time. Since there is no information about the dissolution media, the initial dissolution can be selected based on the experience or other criteria.

6. Calculate recommended $P$ value according to the following Equation 4-6.

7. Measure a series of $\mathrm{P}$ values by varying the conditions in the dissolution media. For example, select different amount of surfactant to increase the drug release rate.

8. Select second dissolution medium based on the recommended $\mathrm{P}$ value and run second in vitro experiment to verify the results.

9. Calculate new $\mathrm{K}$ value from second dissolution study by plotting $\mathrm{Ln}\left[\mathrm{C}_{0} \mathrm{~V}_{0} /\left(\mathrm{C}_{0} \mathrm{~V}_{0}-\mathrm{M}_{\mathrm{t}}\right)\right]$ versus time. If the ratio of $\mathrm{P}$ values between oil/initial medium and oil/second medium has significant difference or $\mathrm{K}$ values are not close to 1 , the new $\mathrm{K}$ value can be used to calculate the recommended $\mathrm{P}$ and select third dissolution medium. Although third dissolution may be performed, it will be the last dissolution study since second dissolution study has narrow down the variance of $K$.

Accelerated study should not change the drug release mechanism and ideally the drug release data in accelerated study can correlate with in vivo drug absorption data. With this assumption, in vivo fraction of drug absorbed $(\mathrm{Fa})$ profile could be considered as the ideal real-time in vitro drug release profile. Unfortunately the IVIVC for parenterals is always the challenge. Chapter $\mathbf{2}$ demonstrated that novel cylinder-inbasket (CIB) in vitro dissolution model has the character of level A IVIVC for parenteral oily formulations. So in this study in vivo drug absorption profiles of tested formulations were used as the real-time in vitro drug release dissolution profiles. All the in vitro dissolution data generated were then compared with the real-time in vitro dissolution profiles in this study and relationship between accelerated in vitro release data and realtime release data were described by Equation 4-3.

$$
\text { Slope }_{\text {in vitro }}=\text { Slope }_{\text {real-time }} \cdot \text { time scale factor }=\text { Slope }_{\text {in vivo }} \cdot \text { time scale factor } \quad(\mathbf{E q . ~ 4 - 3})
$$

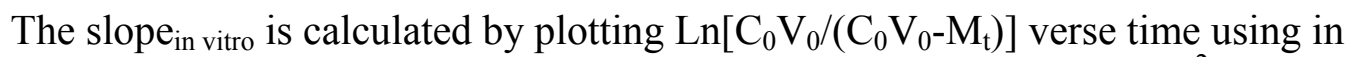
vitro dissolution data and the slope $\mathrm{in}_{\text {in vitro }}\left(\mathrm{K}_{\text {app }}\right)$, coefficient of determination $\left(\mathrm{R}^{2}\right)$ and $\mathrm{K}$ were listed in Table 4-2. The slope of the in vivo profile $\left(\mathrm{Slope}_{\text {in vivo }}\right)$ by plotting $\operatorname{Ln}\left[\mathrm{M}_{\text {total }} /\left(\mathrm{M}_{\text {total }}-\mathrm{M}_{\mathrm{t}}\right)\right]$ verse time follows the first order kinetics and equals to the slope realtime when the time scale factor is one. In this study, the bioavailability of the tested formulations equals to $100 \%$ (chapter 2). Therefore $\mathrm{M}_{\text {total }}$ equals to the amount of drug injected.

From Equation 4-1 and 4-3, parameters of K, time scale factor and $\mathrm{P}$ can be calculated by Equations 4-4, 4-5 and 4-6, respectively.

$$
\text { Slope }_{\text {in vitro }}=\text { Slope }_{\text {real-time }} \cdot \text { Time scale factor }=\mathrm{K}_{\text {app }}=\frac{\mathrm{KS}}{\mathrm{V}_{0} \mathrm{P}}
$$




$$
\begin{gathered}
\mathrm{K}=\frac{\mathrm{K}_{\text {app }} \mathrm{V}_{0} \mathrm{P}}{S} \\
\text { Time scale factor }=\frac{\mathrm{K}_{\text {app }}}{\text { Slope }_{\text {real-time }}} \\
\mathrm{P}=\frac{\mathrm{KS}}{\mathrm{V}_{0} \cdot \text { Slope }_{\text {real-time }} \cdot \text { Time scale factor }}
\end{gathered}
$$

The following two cases will discuss how this rational method is applied to select dissolution medium in which desired drug release rate can achieve.

\subsubsection{Case studies}

\subsubsection{Case 1 (Formulation 4-1, time scale factor $=7$ )}

More than $90 \%$ of drug was released from formulation 4-1 within two weeks (Figure 3-9a). Chapter 3 discussed Level A IVIVC is exist in this dissolution model for the tested formulations. Therefore in vivo percentage of drug absorption of formulation 4-1 (Figure 3-9b) is considered as the ideal in vitro drug release profile. In order to accelerate the in vitro drug release rate of the formulation, time scale factor 7 is applied and the whole process can be described by two steps.

Step 1: calculation of parameter K. In the preliminary study, the solubility of buprenorphine in the medium with either non-ionic (Tween 80; Span 80) or ionic (Sodium Dodecyl Sulfate, SDS) surfactant are tested. The solubility of buprenorphine in 8\% (v/v) Tween 80 in PBS at pH 7.4 and 8\% Span 80 (v/v) in PBS at pH 7.4 are less than $35 \mu \mathrm{g} / \mathrm{mL}$, while the solubility of buprenorphine in PBS with $0.05 \%$ SDS (w/v) at $\mathrm{pH} 7.4$ is $43.8 \mu \mathrm{g} / \mathrm{mL}$. In order to keep the sink condition, the solubility of the buprenorphine in the dissolution medium is desired to be more than $35 \mu \mathrm{g} / \mathrm{mL}$. Higher amount of non-ionic surfactant may also cause larger variation and has less flexibility to adjust in the future study, so surfactant SDS is used in the study to increase the solubility and maintain the sink condition in this study. The experimental $\mathrm{K}_{\mathrm{app}}$ equals to $0.0269 \mathrm{hr}^{-1}$ when the formulation 4-1 is diffused into the medium of PBS with $0.05 \%(\mathrm{w} / \mathrm{v}) \mathrm{SDS}$ at $\mathrm{pH} 6$ (Table 4-2). The cylinder with the diameter of $15 \mathrm{~mm}$ was used in this study and the loading volume $\left(\mathrm{V}_{0}\right)$ of $0.2 \mathrm{~mL}$ was kept the same with the in vivo study. The experimental $\mathrm{P}$ equals to 139.6. Therefore, useful parameter $\mathrm{K}$ can also be calculated and the value is $0.4501 \mathrm{~cm} / \mathrm{hr}$.

Step 2: dissolution medium selection based on tested $\mathrm{P}$ value. It is possible to select the medium with desired $P$ and achieve the objective if all the parameters in Equation 4-6 are available. The aforementioned assumption is if the variance of $\mathrm{P}$ is ignorable, the $\mathrm{K}$ could be considered as a constant to anticipate the $\mathrm{P}$ of the desired dissolution medium. In this case, the calculated $\mathrm{K}$ is around $0.45 \mathrm{~cm} / \mathrm{hr}$ and the calculated $\mathrm{P}$ value is 48.5 from Equation 4-6 with the time scale factor of 7 . After testing a series of 
partition coefficients between ATBC and PBS solution at $\mathrm{pH} 6$ with different amount of SDS, the medium of PBS with $0.1 \%(\mathrm{w} / \mathrm{v})$ SDS at $\mathrm{pH} 6$ was selected to do further dissolution test since tested $\mathrm{P}$ is 47 which is very close to estimated value of 48.5. And the ratio 3.34 of two $P$ values $(139.6 / 41.7)$ is considered as the acceptable variance. The used cylinder has the diameter of $15 \mathrm{~mm}$ and the loading volume of $0.2 \mathrm{~mL}$ was kept the same with the in vivo study. Figure 4-3a indicates the experimental and real-time amount of drug release data. The $\mathrm{x}$ axis is the real-time divided by time scale factor. Figure 4-3b shows that the relationship between accelerated drug release data (\%released $\mathrm{in}_{\text {intro }}(\mathrm{y})$ ) and real-time drug release data divided by time scale factor (\%released real-time $(\mathrm{x}))$ can be described by the linear equation: $y=1.0509 x+0.4309\left(R^{2}=0.9987\right)$. It indicates that the accelerated drug release amount of formulation 4-1 in CIB model can be predicted and a good IVIVC between in vivo drug release and accelerated experimental data is established.

\subsubsection{Case 2 (Formulation 4-2, time scale factor $=14)$}

In vivo drug release of formulation 4-2 can also last two weeks (Figure 3-9a) and in vivo percentage of drug absorption of formulation 4-2 (Figure 3-9b) is considered as the ideal in vitro drug release profile. In order to accelerate the in vitro drug release rate of the formulation, time scale factor 14 is applied and the whole process can be described by two steps.

Step 1: calculation of K. In order to validate aforementioned method, formulation 4-2 was tested at different conditions. The first in vitro dissolution test was performed at $150 \mathrm{rpm}$ in the PBS medium with $0.1 \%(\mathrm{w} / \mathrm{v}) \mathrm{SDS}$ at $\mathrm{pH}$ 7.4. The experimental $\mathrm{P}$ value of buprenorphine between TBC and dissolution medium is 3979.5 . The cylinder with the diameter of $15 \mathrm{~mm}$ and the loading volume $\left(\mathrm{V}_{0}\right)$ of $0.2 \mathrm{~mL}$ were kept the same with the in vivo study. After plotting $\operatorname{Ln}\left[\mathrm{C}_{0} \mathrm{~V}_{0} /\left(\mathrm{C}_{0} \mathrm{~V}_{0}-\mathrm{M}_{\mathrm{t}}\right)\right]$ verse time, the $\mathrm{K}_{\text {app }}$ value is $0.0119 \mathrm{hr}^{-1}$ with $\mathrm{R}^{2}$ of 0.9978 . Therefore the calculated $\mathrm{K}$ is $5.43 \mathrm{~cm} / \mathrm{hr}$ (Table 4-2).

Step 2: selection of dissolution medium based on tested $\mathrm{P}$ value. The sustained release formulation 4-2 can release more than 90\% drug molecules in rats within 14

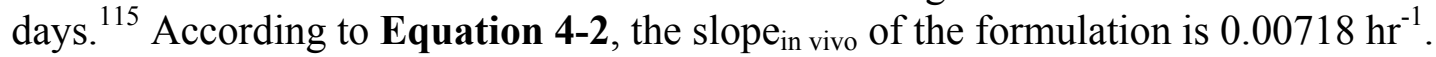
When time scale factor 14 is applied, the recommended $\mathrm{P}$ is 479.74 calculated from Equation 4-6. After testing a series of partition coefficients between TBC and PBS solution at $\mathrm{pH} 7.4$ with different amount of SDS, the medium of PBS with $0.2 \%(\mathrm{w} / \mathrm{v})$ $\mathrm{SDS}$ at $\mathrm{pH} 7.4$ was selected to do further dissolution test since experimental $\mathrm{P}$ is 325 which is close to estimated value. After analyzing the results from the dissolution study, $\mathrm{K}$ value from the new dissolution medium is calculated and the value is 3.53 which is not close to the initial $\mathrm{K}$ value of 5.43. The reason is that the change of the $\mathrm{P}$ values between the two dissolution media (from 3979.5 to 324.6) is beyond the limit of the assumption. Therefore the new recommended P should be calculated from the new $\mathrm{K}$ value, 3.53. The second recommended $\mathrm{P}$ is calculated from the new dissolution results and the $\mathrm{P}$ value is 318 which is close to the previous tested value of 325 . Otherwise, new dissolution medium should be selected based on the tested $\mathrm{P}$ values and the third dissolution test 


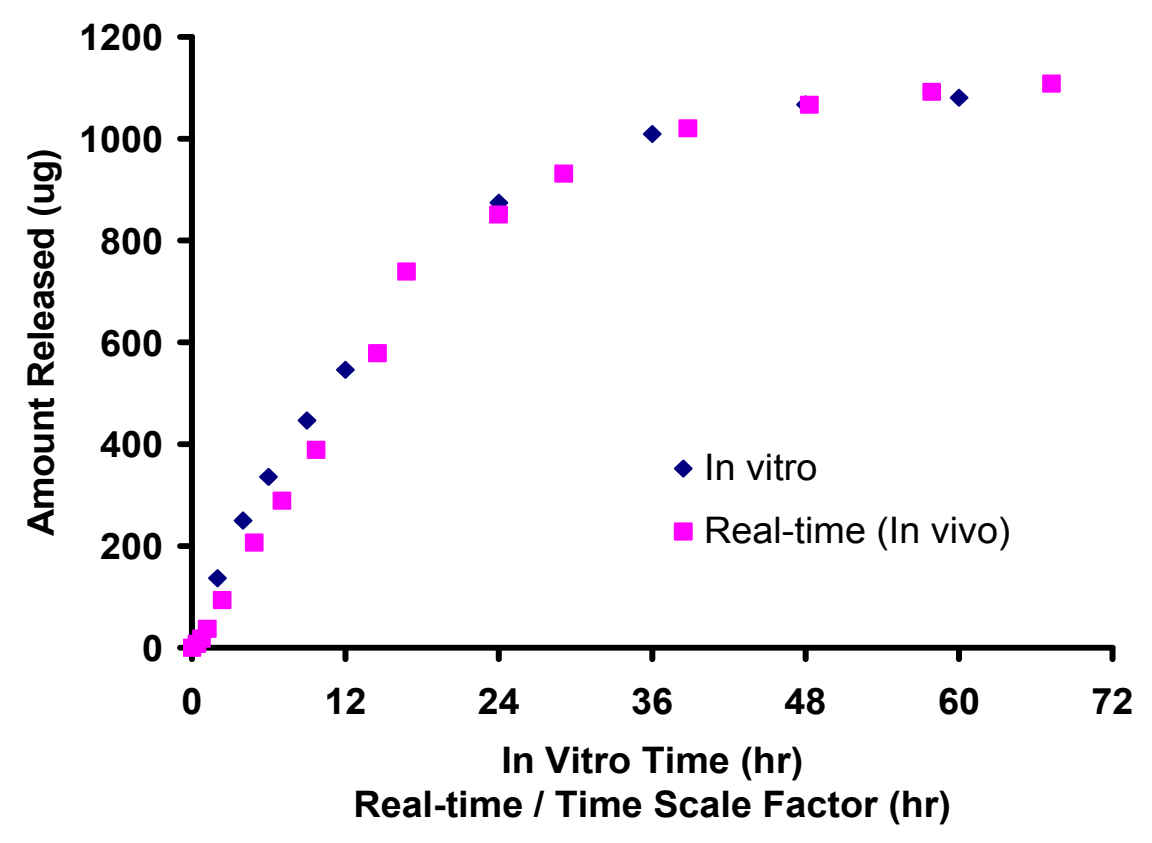

(a)

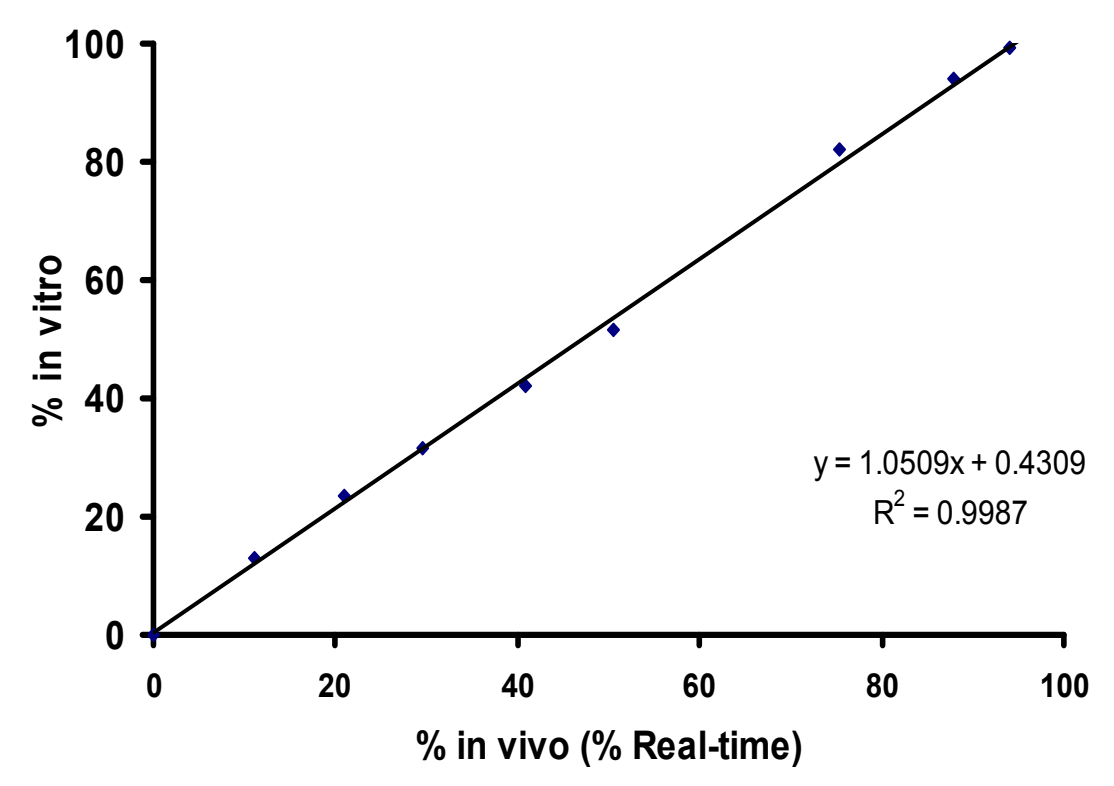

(b)

Figure 4-3. Predicted drug release amount in accelerated study and IVIVC (case 1).

(a) Relationship of drug release amount between experimental and real-time data,

(b) Relationship between $\%$ released $_{\text {in vitro }}(\mathrm{y})$ and $\%$ released $_{\text {real-time }}(\mathrm{x})$ for formulation 4-1. 
should be run and will give desired drug release profile. Figure 4-4a indicates the experimental and real-time amount of drug release. The $\mathrm{x}$-axis is the dissolution time for in vitro study and real-time divided by time scale factor for in vivo study. Figure 4-4b shows that the linear equation, $y=0.9722 x+4.8577\left(R^{2}=0.9931\right)$, can describe the relationship between drug release data in accelerated study (\%released in vitro $\left._{(y)}\right)$ and realtime drug release data $\left(\%\right.$ released $\left._{\text {real-time }}(\mathrm{x})\right)$ by applying time scale factor. It also indicates that a good IVIVC has been established and the drug release amount from formulation 4-2 at different time points in CIB model can be predicted.

\subsubsection{Discussion}

This study presented a rational method to select dissolution medium by testing just one or two in vitro dissolution experiments and measuring the partition coefficients between oily phase and different dissolution media. The initial in vitro dissolution experiment is to calculate $\mathrm{K}$ value of the initial dissolution system and obtain desired $\mathrm{P}$ value. The desired dissolution medium can be selected based on this $\mathrm{P}$ value. The above two cases indicate that the rational method has been developed and established to find the desired dissolution medium.

The assumption of the method is "slightly difference" between the $\mathrm{P}$ values and $\mathrm{K}$ is considered as the constant by changing the content of the medium. In the $2^{\text {nd }}$ case, the ratio of $\mathrm{P}$ values between oil/initial medium and oil/selected medium is 12.3 folds. The predicted $\mathrm{P}$ is 400.8 and the experimental $\mathrm{P}$ value between TBC and selected medium is 325. The tested K values for the two media are close (4.56 vs 3.53, Table 4-2) but can't be rounded. In this case, an additional dissolution experiment may be required to precisely predict $\mathrm{P}$ if ratio of $\mathrm{P}$ values between oil/initial medium and oil/selected medium is too high, or predicted accelerated data does not match the experimental data. It is difficult to define a constant value of $\mathrm{P}$ ratio to be the boundary so far. From author's opinion, comparing $\mathrm{K}$ values may be more practical. If the ratio of $\mathrm{K}$ is close to 1 , only one dissolution experiment can give a good or reasonable prediction. If not, extra attention should be paid and the calculated new $\mathrm{K}$ value from $2^{\text {nd }}$ dissolution medium could be used to calculate new recommended $\mathrm{K}$. The same procedure can be applied again to select dissolution medium. The new dissolution experiment actually refined the dissolution condition. General after maximum two dissolution tests the desired dissolution medium could be found. It is recommended the ratio of $\mathrm{K}$ values should always be examined.

The real-time drug release from formulation 4-1 and 4-2 can last for 14 days. The aforementioned two cases gave the examples how to select the dissolution medium by a rational method to achieve drug release within 2 and 1 day, respectively, without changing the release mechanism. Ideally, shorter dissolution time, such as couple of hours, is preferred in accelerated study to provide the drug release information of the 14 days sustained release formulation, and it will accelerate the formulation development and benefit QC. Theoretically it seems possible to achieve the objective since high amount of surfactant and/or low $\mathrm{pH}$ of dissolution medium could increase the drug 


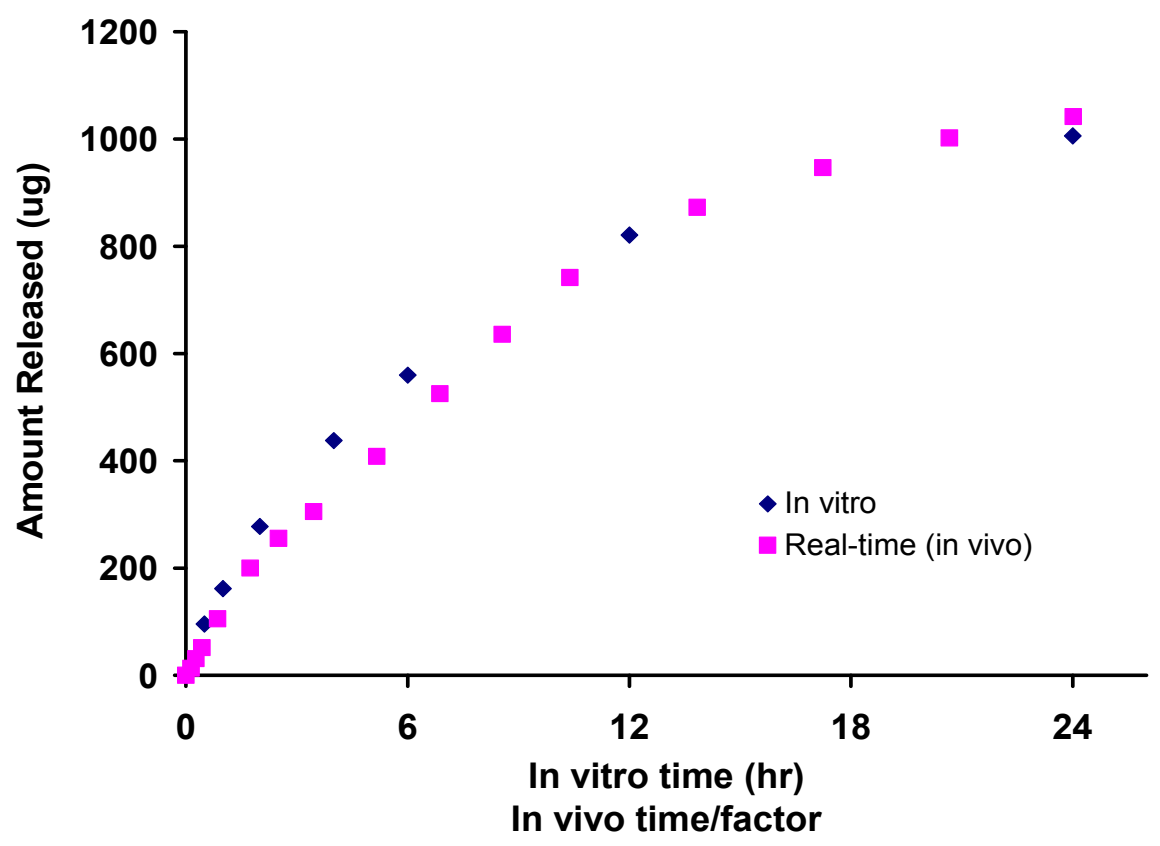

(a)

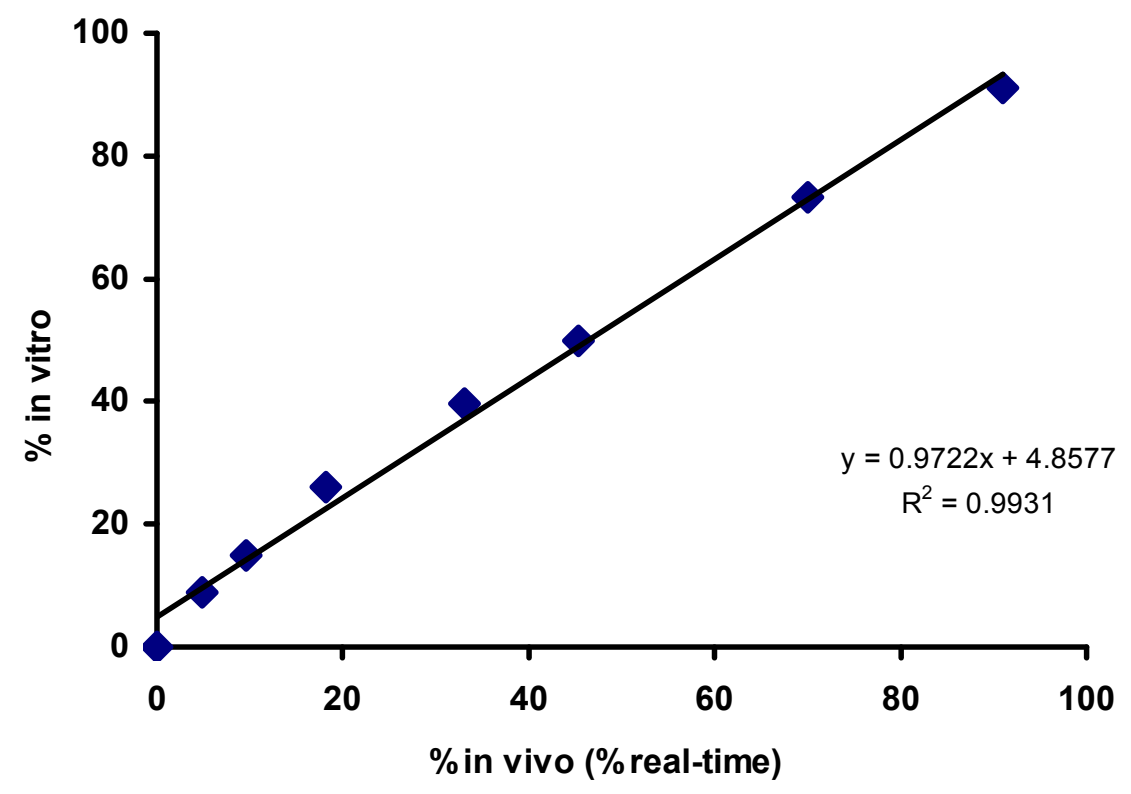

(b)

Figure 4-4. Predicted drug release amount in accelerated study and IVIVC (case 2).

(a) Relationship of drug release amount between experimental and real time data,

(b) Relationship between $\%$ released $_{\text {in vitro }}(\mathrm{y})$ and $\%$ released $_{\text {real-time }}(\mathrm{x})$ for formulation 4-2. 
release rate. Therefore drug release studies in phosphate buffer saline solutions (PBS) with high amount of surfactant at different $\mathrm{pH}$ were performed. The drug release of formulation 4-1 and $4-2$ in PBS with $0.05 \%, 0.1 \%$ or $0.8 \%$ SDS (w/v) at pH $6(50 \mathrm{rpm}$, Figure 4-5) and with $0.1,0.2$ or $0.8 \%$ SDS (w/v) at $\mathrm{pH} 7.4$ (150 rpm, Figure 4-6) were invested. The experiments were performed in triplet. Figure 4-5 and 4-6 indicate that drug release rate increased with the high amount of surfactant in the tested formulations. Good linear relationship $\left(\mathrm{R}^{2}>0.98\right.$, Table 4-2) was obtained in all dissolution media except in the PBS medium with $0.8 \%$ SDS $(\mathrm{w} / \mathrm{v})$ at $\mathrm{pH} 6\left(\mathrm{R}^{2}=0.856\right.$ for formulation 4$1)$. High amount of surfactant $(0.8 \%$ SDS $)$ in the dissolution medium not only dramatically increased the drug release rate, but also caused the large variance (SD, $\sim 20 \%$ ) for certain formulation. During the procedure of loading dissolution medium, it is inevitable to cause some variance when two liquid phases are contacting to each other. Tiny variance between different vessels could be amplified when the drug release rate is significantly increased. This indicates that time scale factor should be carefully selected in order to reduce the variance. The faster release rate, the more variance may get.

\subsection{Conclusions}

Accelerated dissolution testing of buprenorphine oily sustained release formulations was attained by varying the $\mathrm{pH}$ and the amount of the surfactant in the dissolution medium in CIB dissolution model. This study demonstrated that dissolution of oily formulations in USP phosphate buffer with the $\mathrm{pH}$ from 6 to 7.4 followed $1^{\text {st }}$-order kinetic and could be described by Equation 4-1. A rational method (Figure 4-2) was developed in this study to select dissolution medium in the accelerated study according to the practical requirement. The two cases of 14-day sustained release formulations demonstrated how to utilize this method to select dissolution medium to achieve desired in vitro drug release rate without changing drug release mechanism. The proposed method 


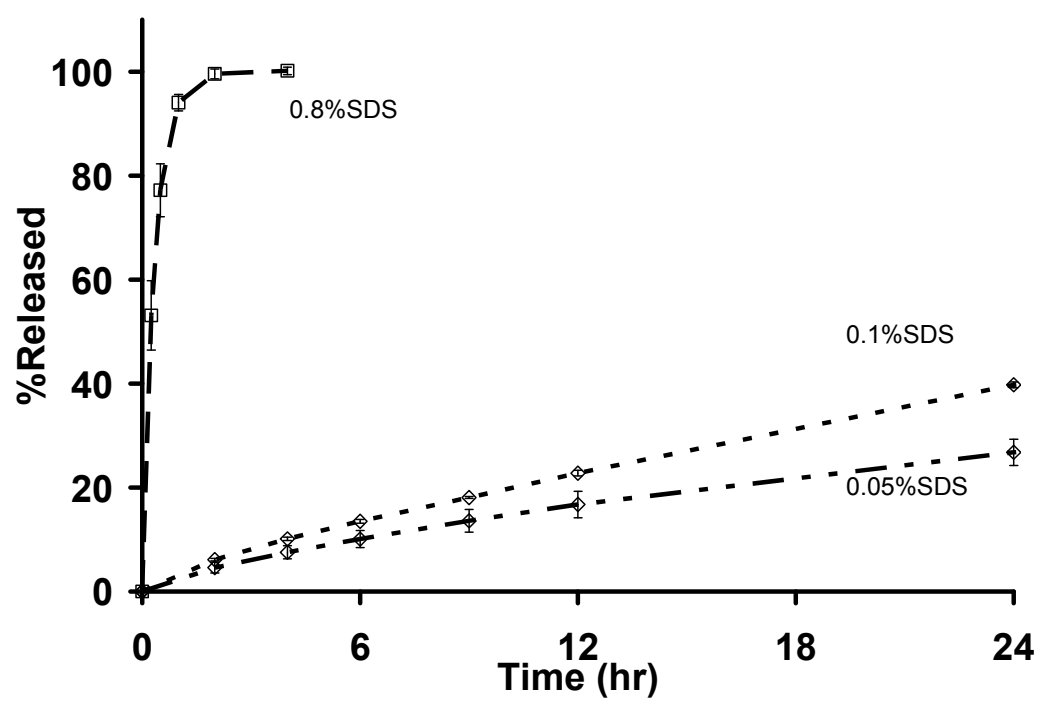

(a)

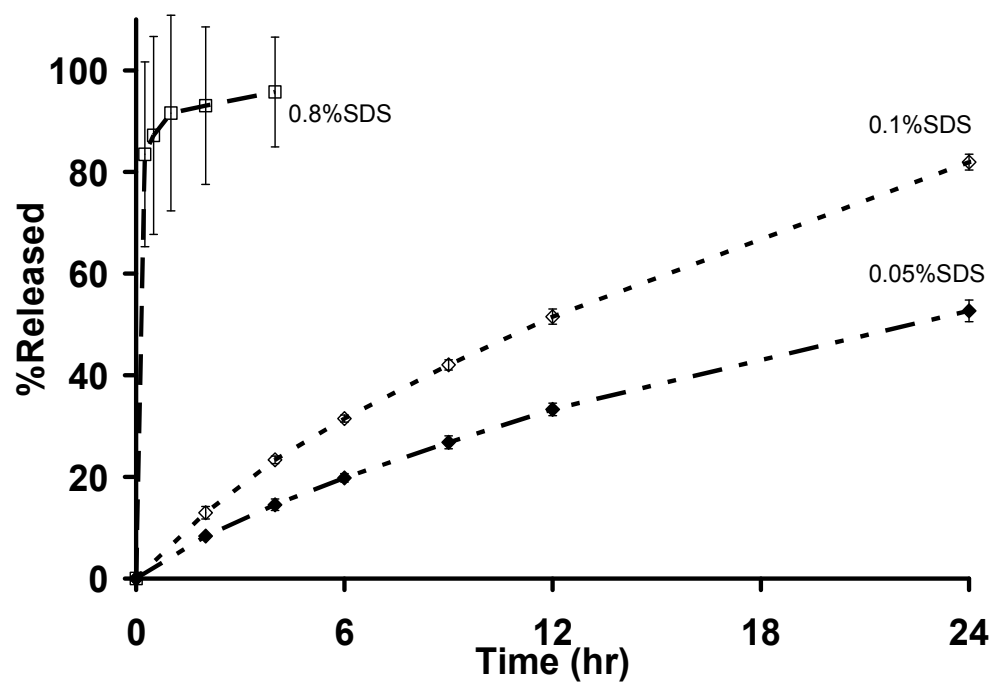

(b)

Figure 4-5. In vitro drug release from (a) formulation 4-1 and (b) formulation 4-2 in phosphate buffer dissolution media with different amount of SDS at pH 6.

$\mathrm{n}=3$.

Data are presented as mean $\pm \mathrm{SD}$. 


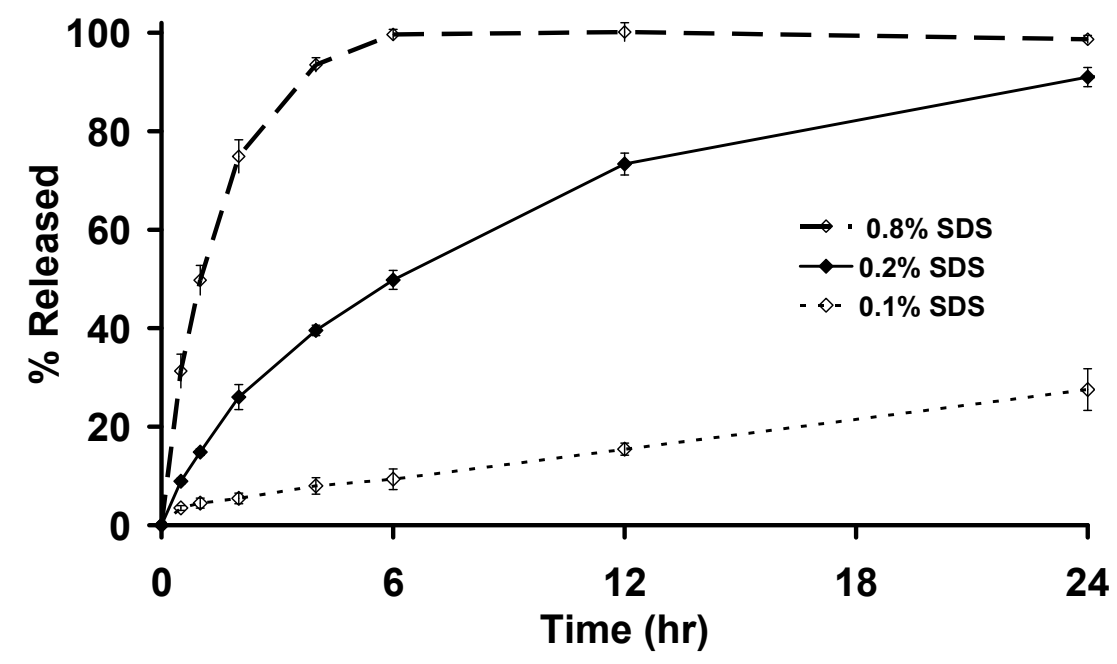

(a)

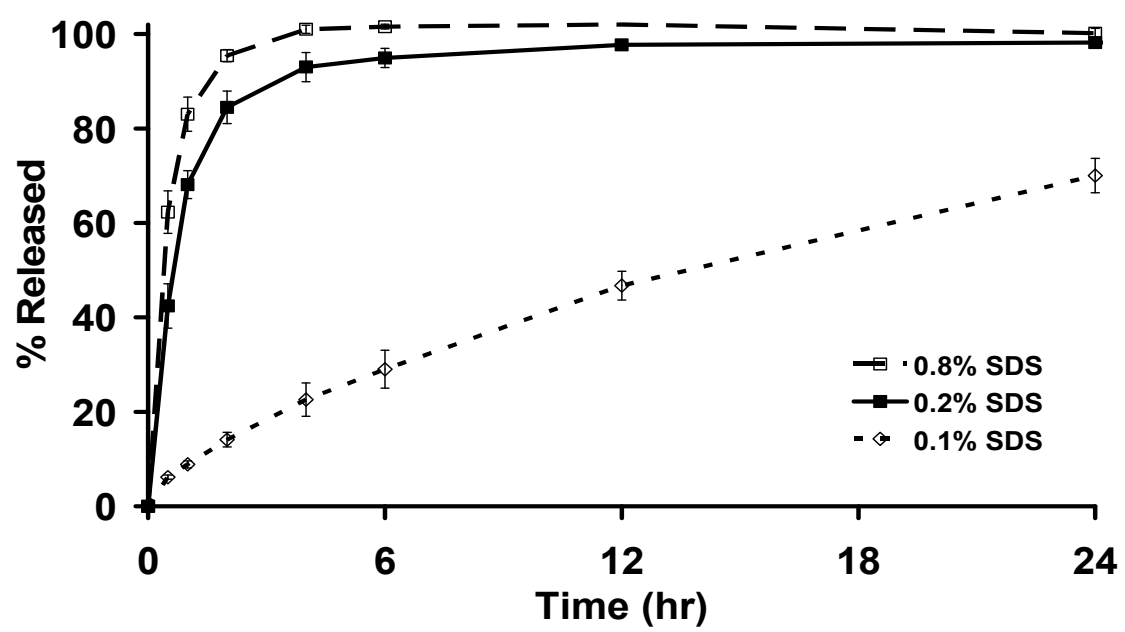

(b)

Figure 4-6. In vitro drug release from (a) formulation 4-1 and (b) formulation 4-2 in phosphate buffer dissolution media with different amount of SDS at pH 7.4.

$\mathrm{n}=3$.

Data are presented as mean $\pm \mathrm{SD}$. 


\section{LIST OF REFERENCES}

1. U.S. Food and Drug Administration. http://www.fda.gov/default.htm. Accessed October 19, 2011.

2. Ohtani M 2007. Basic pharmacology of buprenorphine. 1(1):69-73.

3. BuTrans (Buprenorphine) Transdermal System: Chemistry reviews. http://www.accessdata.fda.gov/drugsatfda_docs/nda/2010 /021306Orig1s000ChemR.pdf. Accessed Oct 17, 2011.

4. Likar R 2006. Transdermal buprenorphine in the management of persistent pain safety aspects. Ther Clin Risk Manag 2(1):115-125.

5. Norspan Transdermal Patch Label. http://secure.healthlinks.net.au/content/mf /pi.cfm?product=mfpnorsp. Accessed October 19, 2011.

6. Titan Pharmaceuticals, Inc.: Products and technologies. http://www.titanpharm.com/products.htm\#gpm1_1. Accessed October 19, 2011.

7. Cowan A 2007. Buprenorphine: the basic pharmacology revisited. J Addict Med 1(2):68-72.

8. Cowan A, Lewis JW, Macfarlane IR 1977. Agonist and antagonist properties of buprenorphine, a new antinociceptive agent. Br J Pharmacol 60(4):537-545.

9. Lizasoain I, Leza JC, Lorenzo P 1991. Buprenorphine: bell-shaped dose-response curve for its antagonist effects. Gen Pharmacol 22(2):297-300.

10. Leander JD 1988. Buprenorphine is a potent kappa-opioid receptor antagonist in pigeons and mice. Eur J Pharmacol 151(3):457-461.

11. Gilbert PE, Martin WR 1976. The effects of morphine and nalorphine-like drugs in the nondependent, morphine-dependent and cyclazocine-dependent chronic spinal dog. J Pharmacol Exp Ther 198(1):66-82.

12. Cowan A, Doxey JC, Harry EJR 1977. The animal pharmacology of buprenorphine, an oripavine analgesic agent. Br J Pharmacol 60(4):547-554.

13. Cowan A, Lewis JW, Macfarlane IR 1977. Agonist and antagonist properties of buprenorphine, a new antinociceptive agent. Br J Pharmacol 60(4):537-545.

14. Lutfy K, Eitan S, Bryant CD, Yang YC, Saliminejad N, Walwyn W, Kieffer BL, Takeshima H, Carroll FI, Maidment NT, Evans CJ 2003. Buprenorphine-induced antinociception is mediated by mu-opioid receptors and compromised by concomitant activation of opioid receptor-like receptors. J Neurosci 23(32):10331-10337. 
15. Cowan A, Lewis JW 1995. Buprenorphine: Combatting Drug Abuse with a Unique Opioid. Wiley-Liss:113-136.

16. Richards ML, Sadee W 1985. Buprenorphine is an Antagonist at the $\kappa$ Opioid Receptor. (4):178-181.

17. Lutfy K, Cowan A 2004. Buprenorphine: A unique drug with complex pharmacology. Curr Neuropharmacol 2(4):395-402.

18. Zhang W, Ramamoorthy Y, Tyndale RF, Sellers EM 2003. Interaction of buprenorphine and its metabolite norbuprenorphine with cytochromes p450 in vitro. Drug Metab Dispos 31(6):768-772.

19. Budd K 1981. High dose buprenorphine for postoperative analgesia. Anaesthesia 36(9):900-903.

20. Cowan A, Lewis JW, Macfarlane IR, Whittle BA 1971. Analgesic and dependence studies with oripavine partial agonists. Br J Pharmacol 43(2):461P$462 \mathrm{P}$.

21. Huang P, Kehner GB, Cowan A, Liu-Chen L-Y 2001. Comparison of pharmacological activities of buprenorphine and norbuprenorphine: norbuprenorphine is a potent opioid agonist. J Pharmacol Exp Ther 297(2):688695.

22. Negus SS, Bidlack JM, Mello NK, Furness MS, Rice KC, Brandt MR 2002. Delta opioid antagonist effects of buprenorphine in rhesus monkeys. Behav Pharmacol 13(7):557-570.

23. Ohtani M, Kotaki H, Uchino K, Sawada Y, Iga T 1994. Pharmacokinetic analysis of enterohepatic circulation of buprenorphine and its active metabolite, norbuprenorphine, in rats. Drug Metab Dispos 22(1):2-7.

24. Gopal S, Tzeng T-B, Cowan A 2002. Characterization of the pharmacokinetics of buprenorphine and norbuprenorphine in rats after intravenous bolus administration of buprenorphine. Eur J Pharm Sci 15(3):287-293.

25. Yu S, Zhang X, Sun Y, Peng Y, Johnson J, Mandrell T, Shukla AJ, Laizure SC 2006. Pharmacokinetics of buprenorphine after intravenous administration in the mouse. J Am Assoc Lab Anim Sci 45(3):12-16.

26. Harris D, Robinson JR 1992. Drug delivery via the mucous membranes of the oral cavity. J Pharm Sci 81(1):1-10.

27. Brewster D, Humphrey MJ, McLeavy MA 1981. The systemic bioavailability of buprenorphine by various routes of administration. J Pharm Pharmacol 33(8):500-506. 
28. Yu S 2006. Development of a sustained release injectable suspension dosage form of buprenorphine. Dissertation (7-8).

29. Pontani RB, Vadlamani NL, Misra AL 1985. Disposition in the rat of buprenorphine administered parenterally and as a subcutaneous implant. Xenobiotica 15(4):287-297.

30. Brewster D, Humphrey MJ, McLeavy MA 1981. Biliary excretion, metabolism and enterohepatic circulation of buprenorphine. Xenobiotica 11(3):189-196.

31. Cone EJ, Gorodetzky CW, Yousefnejad D, Buchwald WF, Johnson RE 1984. The metabolism and excretion of buprenorphine in humans. Drug Metab Dispos 12(5):577-581.

32. Rat Medicantion Guide. http://ratguide.com/meds/analgesics /narcotic analgesics.php. Accessed October 19, 2011.

33. Adams HR 2001. Veterinary Pharmacology and Therapeutics, 8th.268-298.

34. Clark MD, Krugner-Higby L, Smith LJ, Heath TD, Clark KL, Olson D 2004. Evaluation of liposome-encapsulated oxymorphone hydrochloride in mice after splenectomy. Comp Med 54(5):558-563.

35. Stokes EL, Flecknell PA, Richardson CA 2009. Reported analgesic and anaesthetic administration to rodents undergoing experimental surgical procedures. Lab Anim 43(2):149-154.

36. Rat Medicantion Guide. http://ratguide.com/meds/analgesics /buprenorphine hydrochloride.php. Accessed October 19, 2011.

37. Guidelines for Analgesics: Mouse. http://vetmed.duhs.duke.edu /guidelines for analgesics mouse.htm. Accessed October 19, 2011.

38. Animal Analgesic Regimens: Rats. http://research.uiowa.edu/animal /index.php?get=aa regimens rats. Accessed October 19, 2011.

39. Analgesic Drugs. http://www.dar.emory.edu/forms/Vet/Analgesic_drugs 05.pdf. Accessed October 19, 2011.

40. Animal Analgesic Regimens: Mice. http://research.uiowa.edu/animal/index.php ?get $=$ aa_regimens_mice. Accessed October 19, 2011.

41. Flecknell PA 1999. Comparison of the effects of oral or subcutaneous carprofen or ketoprofen in rats undergoing laparotomy. Veterinary Record 144: 65-67.

42. Guidelines for Rodent Analgesia. http://vetmed.duhs.duke.edu /guidelines_for_rodent analgesia.htm. Accessed October 19, 2011. 
43. Current Recommendations for Analgesia in Rats and Mice.

http://www.google.com/url?sa=t\&source=web\&ct=res\&cd=1\&ved $=0 \mathrm{CAwQFjAA} \& u r \mathrm{~h}=\mathrm{http} \% 3 \mathrm{~A} \% 2 \mathrm{~F} \% 2 \mathrm{Fwww}$.ahc.umn.edu $\% 2 \mathrm{FRAR}$ \%2FAcetaminophenUpdate.doc\&rct $=\mathrm{j} \& \mathrm{q}=\mathrm{Current}+$ recommendations + for + analgesia + in + rats + and + mice\&ei $=$ UzYZS-WqG4a1tge7sdnYAw\&usg =AFQjCNENeGePtig GAIYqumS5MeuPIQjUQ. Accessed October 19, 2011.

44. Gray RE, Munks MW, Haynes RR, Olsen GD 2001. Mu opioid receptor efficacy and potency of morphine-6-glucuronide in neonatal guinea pig brainstem membranes: comparison with transfected CHO cells. Brain Res Bull 54(5):499505.

45. Drugs: Morphine sulfate injection. http://www.drugs.com/pro/morphineinjection.html. Accessed October 19, 2011.

46. Rat Medicine Guide. http://ratguide.com/meds/analgesics butorphanol tartrate.php. Accessed October 19, 2011.

47. ACLAM. http://www.aclam.org/print/position_pain-rodent-rabbit.pdf. Accessed October 19, 2011.

48. Cooper DM, Hoffman W, Wheat N, Lee HY 2005. Duration of effects on clinical parameters and referred hyperalgesia in rats after abdominal surgery and multiple doses of analgesic. Comp Med 55(4):344-353.

49. Tramadol. http://en.wikipedia.org/wiki/Tramadol. Accessed October 19, 2011.

50. Rat Medicine Guide. http://ratguide.com/meds/nsaids/carprofen.php. Accessed October 19, 2011.

51. Donald C. Plumb, Veterinary Drug Handbook, 4th Edition, 2002.

52. Acetaminophen. http://www.ahc.umn.edu/RAR/AcetaminophenUpdate.doc. Accessed October 19, 2011.

53. Tramadol Injection. http://www.healthbiotech.in/tramadol-injection.html. Accessed October 19, 2011.

54. Latest Drug Alerts and Warnings. http://healthcare.utah.edu/pharmacy/alerts 356.htm. Accessed October 19, 2011.

55. Rimadyl label. http://www.fda.gov/downloads/AnimalVeterinary/Products /ApprovedAnimalDrugProducts/DrugLabels/UCM050410.pdf. Accessed October 19, 2011. 
56. Meloxicam. http://www.google.com/url?sa=t\&source=web\&ct=res\&cd=1\&ved $=0 \mathrm{CAgQFjAA \& url=http} \% 3 \mathrm{~A} \% 2 \mathrm{~F} \% 2 \mathrm{Fwww} \cdot$ ahc.umn.edu $\% 2 \mathrm{FRAR}$

\%2FAcetaminophenUpdate.doc\& $\mathrm{rct}=\mathrm{j} \& \mathrm{q}=$ Meloxicam+rats\&ei

=8DIZS7PhO8qutgeNrqTdAw\&usg=AFQjCNENeGePtig_GAIYqumS5MeuP1Qj UQ. Accessed October 19, 2011.

57. Metacam Solution Injection: Cats. http://www.bi-vetmedica.com/product /metacam-solution-injection-cats. Accessed October 19, 2011.

58. Ketoprofen Injection. http://www.horsemedsonline.com /ketoprofen-injection-50ml-p-48.html. Accessed October 19, 2011.

59. Ketoprofen. http://wildlife1.wildlifeinformation.org/S/00Chem/ChComplex Ketoprofen.htm. Accessed October 19, 2011.

60. Drugs: Anafen injection. http://74.125.47.132/search

?q=cache:I38gzVhsv54J:www.drugs.com/vet

/anafen-injection-100-mg-ml-can.html+Ketoprofen + inject\& $\mathrm{cd}=6 \& \mathrm{hl}=\mathrm{en} \& \mathrm{ct}=\mathrm{clnk}$ $\underline{\text { \&l=us\&client }=\text { firefox-a. }}$. Accessed October 19, 2011.

61. Hofmeister EH, Egger CM 2004. Transdermal fentanyl patches in small animals. J Am Anim Hosp Assoc 40(6):468-478.

62. Egger CM, Glerum L, Michelle Haag K, Rohrbach BW 2007. Efficacy and costeffectiveness of transdermal fentanyl patches for the relief of post-operative pain in dogs after anterior cruciate ligament and pelvic limb repair. Vet Anaesth Analg 34(3):200-208.

63. Ahern BJ, Soma LR, Boston RC, Schaer TP 2009. Comparison of the analgesic properties of transdermally administered fentanyl and intramuscularly administered buprenorphine during and following experimental orthopedic surgery in sheep. Am J Vet Res 70(3):418-422.

64. Foley PL, Henderson AL, Bissonette EA, Wimer GR, Feldman SH 2001. Evaluation of fentanyl transdermal patches in rabbits: blood concentrations and physiologic response. Comp Med 51(3):239-244.

65. Krugner-Higby L, Smith L, Clark M, Heath TD, Dahly E, Schiffman B, HubbardVanStelle S, Ney D, Wendland A 2003. Liposome-encapsulated oxymorphone hydrochloride provides prolonged relief of postsurgical visceral pain in rats. Comp Med 53(3):270-279.

66. Smith LJ, Krugner-Higby L, Clark M, Wendland A, Heath TD 2003. A single dose of liposome-encapsulated oxymorphone or morphine provides long-term analgesia in an animal model of neuropathic pain. Comp Med 53(3):280-287. 
67. Smith LJ, Valenzuela JR, Krugner-Higby LA, Brown C, Heath TD 2006. A single dose of liposome-encapsulated hydromorphone provides extended analgesia in a rat model of neuropathic pain. Comp Med 56(6):487-492.

68. Foley PL, Liang H, Crichlow AR 2011. Evaluation of a sustained-release formulation of buprenorphine for analgesia in rats. Journal of the American Association for Laboratory Animal Science 50(2):198-204.

69. Larsen C, Larsen SW, Jensen H, Yaghmur A, Ostergaard J 2009. Role of in vitro release models in formulation development and quality control of parenteral depots. Expert Opin Drug Deliv 6(12):1283-1295.

70. Krishna R, Yu L 2008. Biopharmaceutics Applications in Drug Development.336-358.

71. Schultz K, Mollgaard B, Frokjaer S, Larsen C 1997. Rotating dialysis cell as in vitro release method for oily parenteral depot solutions. Int J Pharm 157(2):163169.

72. Fotaki N 2011. Flow-Through Cell Apparatus (USPApparatus 4): Operation and Features. Dissolution Technologies 46-49.

73. Ultrafiltration. http://en.wikipedia.org/wiki/Ultrafiltration. Accessed December 14, 2011.

74. Magenheim B, Levy MY, Benita S 1993. A new in vitro technique for the evaluation of drug release profile from colloidal carriers - ultrafiltration technique at low pressure. International Journal of Pharmaceutics 94(1-3):115-123.

75. Dash AK, Haney PW, Garavalia MJ 1999. Development of an in vitro dissolution method using microdialysis sampling technique for implantable drug delivery systems. J Pharm Sci 88(10):1036-1040.

76. Microdialysis. http://en.wikipedia.org/wiki/Microdialysis. Accessed December $14,2011$.

77. FDA 1997. Extended Release Oral Dosage Forms: Development, Evaluation, and Application of In Vitro/In Vivo Correlations. Food and Drug Administration, Rockville, MD.

78. USP 1995. In Vitro and In Vivo Evaluation of Dosage form < 1088>.1824-1929.

79. DEMIRTÜRK E, ÖNER L 2003. In Vitro - In Vivo Correlations. FABAD Journal of Pharmaceutical Sciences 28(4):215-224.

80. Trull FL, Rich BA 1999. More regulation of rodents. Science 284(5419):1463. 
81. Office H 2010. Statistics of Scientific Procedures on Living Animals Great Britain.

82. Cowan A 1976. Use of the mouse jumping test for estimating antagonistic potencies of morphine antagonists. J Pharm Pharmacol 28(3):177-182.

83. Dettmar PW, Cowan A. 1980, pp 173-174.

84. Bryant RM, Olley JE, Tyers MB 1983. Antinociceptive actions of morphine and buprenorphine given intrathecally in the conscious rat. Br J Pharmacol 78(4):659663.

85. Sakai Y 1989. Relation between in vivo opioid receptor binding of buprenorphine and analgesic effect in rat. Kyoto-furitsu Ika Daigaku Zasshi 98(9):949-959.

86. Cowan A 2003. Buprenorphine: New pharmacological aspects. Int J Clin Pract, Suppl 133:3-8.

87. Johnson RE, Fudala PJ, Payne R 2005. Buprenorphine: considerations for pain management. J Pain Symptom Manage 29(3):297-326.

88. Joubert KE 2001. The use of analgesic drugs by South African veterinarians. J S Afr Vet Assoc 72(1):57-60.

89. Capner CA, Lascelles BD, Waterman-Pearson AE 1999. Current British veterinary attitudes to perioperative analgesia for dogs. Vet Rec 145(4):95-99.

90. Roughan JV, Flecknell PA 2002. Buprenorphine: a reappraisal of its antinociceptive effects and therapeutic use in alleviating post-operative pain in animals. Lab Anim 36(3):322-343.

91. Yassen A, Olofsen E, Kan J, Dahan A, Danhof M 2008. Pharmacokineticpharmacodynamic modeling of the effectiveness and safety of buprenorphine and fentanyl in rats. Pharm Res 25(1):183-193. Epub 2007 Oct 2004.

92. Christoph T, Kogel B, Schiene K, Meen M, De Vry J, Friderichs E 2005. Broad analgesic profile of buprenorphine in rodent models of acute and chronic pain. Eur J Pharmacol 507(1-3):87-98. Epub 2004 Dec 2030.

93. Gades NM, Danneman PJ, Wixson SK, Tolley EA 2000. The magnitude and duration of the analgesic effect of morphine, butorphanol, and buprenorphine in rats and mice. Contemp Top Lab Anim Sci 39(2):8-13.

94. Weng Larsen S, Larsen C 2009. Critical factors influencing the in vivo performance of long-acting lipophilic solutions--impact on in vitro release method design. Aaps J 11(4):762-770. Epub 2009 Nov 2006. 
95. Johnson W, Jr. 2002. Final report on the safety assessment of acetyl triethyl citrate, acetyl tributyl citrate, acetyl trihexyl citrate, and acetyl trioctyl citrate. Int J Toxicol 21(Suppl 2):1-17.

96. Rowe RC, Sheskey PJ, Quinn ME 2009. Handbook of Pharmaceutical Excipients.

97. Sadée W, Rosenbaum JS, Herz A 1982. Buprenorphine: differential interaction with opiate receptor subtypes in vivo. J Pharmacol Exp Ther 223(1):157-162.

98. Swedberg MD 1994. The mouse grid-shock analgesia test: pharmacological characterization of latency to vocalization threshold as an index of antinociception. J Pharmacol Exp Ther 269(3):1021-1028.

99. Meyers DB, Autian J, Guess WL 1964. Toxicity of plastics used in medical practice II. Toxicity of citric acid esters used as plasticizers. Journal of Pharmaceutical Sciences 53(7):774-777.

100. Finkelstein M, Gold H 1959. Toxicology of the citric acid esters: Tributyl citrate, acetyl tributyl citrate, triethyl citrate, and acetyl triethyl citrate. Toxicology and Applied Pharmacology 1(3):283-298.

101. Pain Management. http://web.jhu.edu/animalcare/procedures/survivalrodents.html\#pain. Accessed October 19, 2011.

102. Washington C 1989. Evaluation of non-sink dialysis methods for the measurement of drug release from colloids: effects of drug partition. International Journal of Pharmaceutics 56(1):71-74.

103. Martinez M, Rathbone M, Burgess D, Huynh M 2008. In vitro and in vivo considerations associated with parenteral sustained release products: a review based upon information presented and points expressed at the 2007 Controlled Release Society Annual Meeting. J Control Release 129(2):79-87.

104. Larsen DB, Joergensen S, Olsen NV, Hansen SH, Larsen C 2002. In vivo release of bupivacaine from subcutaneously administered oily solution. Comparison with in vitro release. J Control Release 81(1-2):145-154.

105. Larsen SW, Jessen MN, Ostergaard J, Larsen C 2008. Assessment of drug release from oil depot formulations using an in vitro model -- potential applicability in accelerated release testing. Drug Dev Ind Pharm 34(3):297-304.

106. Burgess DJ, Crommelin DJ, Hussain AS, Chen ML 2004. Assuring quality and performance of sustained and controlled release parenterals: EUFEPS workshop report. AAPS J 6(1):100-111.

107. Chilukuri DM, Sunkara G, Young D 2007. Pharmaceutical Product Development In Vitro-In Vivo Correlation. 165:141-152. 
108. FDA 1997. Extended Release Oral Dosage Forms: Development, Evaluation, and Application of In Vitro/In Vivo Correlations. Food and Drug Administration, Rockville, MD

109. Larsen DH, Fredholt K, Larsen C 2000. Assessment of rate of drug release from oil vehicle using a rotating dialysis cell. European Journal of Pharmaceutical Sciences 11(3):223-229.

110. Hirano K, Ichihashi T, Yamada H 1982. Studies on the absorption of practically water-insoluble drugs following injection V: Subcutaneous absorption in rats from solutions in water immiscible oils. J Pharm Sci 71(5):495-500.

111. FDA 1997. Guidance for Industry: Dissolution Testing of Immediate Release Solid Oral Dosage Forms. Food and Drug Administration, Rockville, MD

112. Gibaldi M, Perrier D 1982. Pharmacokinetics. 2nd edition:146-195.

113. Dalton JT, Straughn AB, Dickason DA, Grandolfi GP 2001. Predictive ability of level A in vitro-in vivo correlation for ringcap controlled-release acetaminophen tablets. Pharm Res 18(12):1729-1734.

114. D'Souza SS, DeLuca PP 2006. Methods to assess in vitro drug release from injectable polymeric particulate systems. Pharm Res 23(3):460-474.

115. Xu J, Qu W, Cheng W, Zhou Y, Bronsun E, Mandrell T, Almoazen H, Johnson JR Submitted. A Novel In Vitro Dissolution Model For Oily Formulations: Model design, release mechanism, and the development of Level A IVIVC. Journal of Controlled Release. Submitted.

116. Zolnik BS, Leary PE, Burgess DJ 2006. Elevated temperature accelerated release testing of PLGA microspheres. Journal of Controlled Release 112(3):293-300.

117. Waterbeemd Hvd, Lennernäs H, Artursson P, Mannhold R, Kubinyi H, Folkers G 2003. Drug Bioavailability: Estimation of Solubility, Permeability, Absorption and Bioavailability. Chapter 8:191-214. 


\section{VITA}

Mr. Jin Xu was born in Nanjing, P. R. China in 1979. He graduated with a Bachelor of Science degree from the China Pharmaceutical University in June 2001. Mr. Jin Xu was enrolled in the Medicinal Chemistry program at the University of Tennessee Health Science Center and was rewarded a Graduate Teaching Assistantship in August 2004. He completed his Master degree in 2007. Then he was enrolled in the Doctor of Philosophy program and joined Drs. James R. Johnson and Atul J. Shukla's group in Department of Pharmaceutical Sciences at the University of Tennessee Health Science Center in 2008. Mr. Jin Xu is a member of the Rho Chi Pharmaceutical Honorary Society, the American Association of Pharmaceutical Scientists (AAPS), the Controlled Release Society (CRS) and American Chemical Society (ACS). 